sous la direction

de Julien Devriendt 


\title{
Jouer en bibliothèque
}

\author{
Julien Devriendt (dir.)
}

DOI : 10.4000/books.pressesenssib.4181

Éditeur: Presses de l'enssib

Lieu d'édition : Villeurbanne

Année d'édition : 2015

Date de mise en ligne : 30 janvier 2019

Collection : La Boîte à outils

ISBN électronique : 9782375460719

\section{Sbooks}

http://books.openedition.org

\section{Édition imprimée}

Date de publication : 1 janvier 2015

ISBN : 9791091281546

Nombre de pages : 176

\section{Référence électronique}

DEVRIENDT, Julien (dir.). Jouer en bibliothèque. Nouvelle édition [en ligne]. Villeurbanne : Presses de l'enssib, 2015 (généré le 01 février 2021). Disponible sur Internet : <http://books.openedition.org/ pressesenssib/4181>. ISBN : 9782375460719 . DOI : https://doi.org/10.4000/books. pressesenssib. 4181.

(C) Presses de l'enssib, 2015

Conditions d'utilisation:

http://www.openedition.org/6540 
JOUER EN BIBLIOTHĖQUE

sous la direction de Julien Devriendt 


\section{$\mathrm{BAO \# 34}$}

\section{JOUER EN BIBLIOTHÈQUE}

Le jeu est de plus en plus présent dans les bibliothèques : l'expansion du jeu vidéo comme l'engouement des jeunes adultes pour les jeux de plateau accélèrent leur réinvestissement par les bibliothécaires. Par ailleurs, de nouveaux dispositifs ludiques sont venus prolonger les services traditionnels des ludothèques.

Pratiques culturelles à succès, les jeux concernent tous les publics et sont mobilisés dans plusieurs domaines d'application, comme outils de création, de découverte ou d'apprentissage, ou encore comme supports de divertissement.

Comment permettre la pratique de l'expérience du jeu ? Comment penser le jeu comme une fin et pas seulement un moyen ? Quels espaces, quelles équipes ? Quels sont les services à inventer et organiser ? Comment développer des fonds ? Quel cadre réglementaire établir?

Ce volume apporte des éléments de réponse pratiques, tirés de réalisations concrètes, menées aussi bien à l'université qu'au collège, dans des médiathèques ou des services de lecture départementaux. Quatre axes structurent le plan : Connaître le contexte (les espaces, les équipes, le droit) ; Acquérir-valoriser (jouets, ludo'malles et serious games) ; Animer-créer (projet de service, partenariats, médiation) ; Participer (une approche orientée communauté).

Coordonné par Julien Devriendt, spécialiste du sujet et formateur, cet ouvrage collectif propose un ensemble de repères et de connaissances indispensables pour se lancer dans des projets ludiques, véritablement intégrés à la stratégie de son établissement.

Mots clés : cdi, chasses au trésor, création, enfants, Institut français, jeux en réalité alternée, jeux sérieux, jeux vidéo, ludification, ludomalles, ludothèques, médiation numérique, minecraft, pratiques participatives, québec 


\section{\#24}

JOUER EN

BIBLIOTHĖQUE

Sous la direction de Julien Devriendt 
Ont contribué à cet ouvrage:

\author{
Véronique Amar \\ Anthony Avila \\ Florence Carre \\ Julien Devriendt \\ Catherine Di Sciullo \\ Thomas Fourmeux \\ Sophie Jacob \\ Caroline Makosza
}

\author{
Lionel Maurel \\ Céline Meneghin \\ Thierry Robert \\ Éric Sanchez \\ Guillemette Trognot \\ Marie-Paule Voïta \\ Solenne Waszak \\ Élise Ybled
}

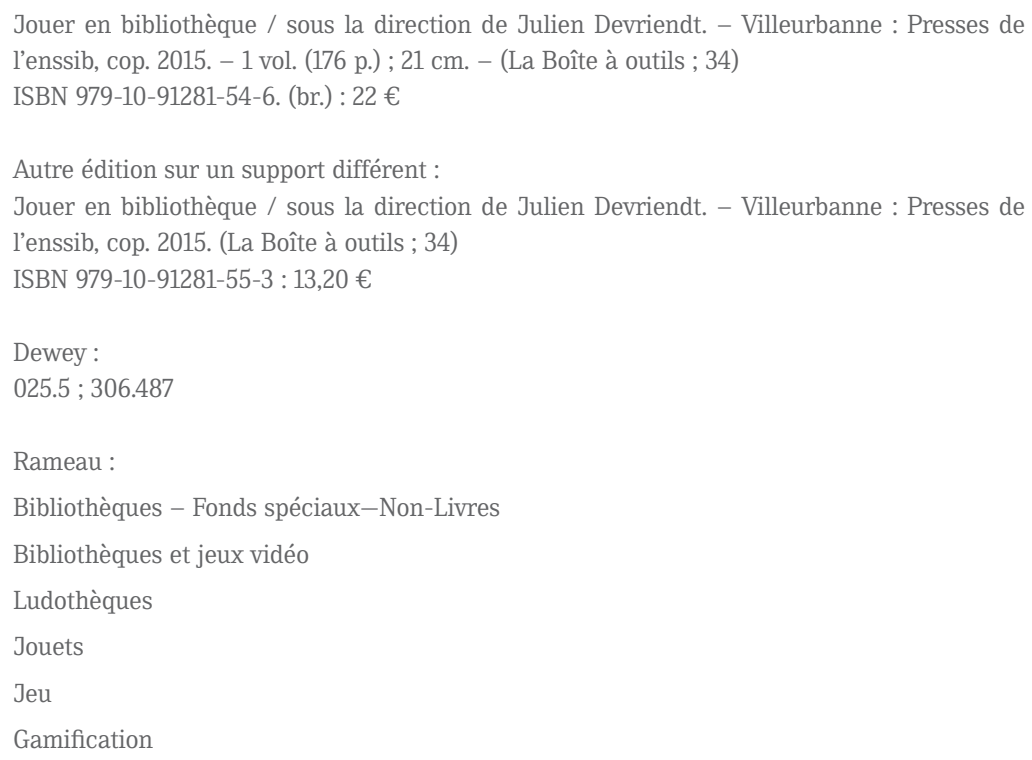




\section{SOMMAIRE}

MODE D'EMPLOI.

9

\section{PARTIE I. CONNAT̂TTE}

LE CONTEXTE.

15

LE JEU COMME BIEN CULTUREL ET COMMUN .16

par Céline Meneghin

JEUX DE SOCIÉTÉ, JEUX ET SOCIÉTÉS .16

UNE ACTIVITÉ SOCIALE

ACCOMPAGNER LES ÉQUIPES .25

LA MÉDIATION NUMÉRIQUE :

UN PROJET D'ÉQUIPE À

LA GAÎTÉ LYRIQUE .26

par Catherine Di Sciullo

LA COMPLÉMENTARITÉ, UNE CLÉ DE RÉUSSITE 26

IDENTIFIER LES SAVOIR-FAIRE NÉCESSAIRES

L'AUTOFORMATION :

UN DYNAMISME À ENCLENCHER ..... 28

LA MISE EN PLACE D'ATELIERS DE DÉCOUVERTE D'APPLICATIONS SUR TABLETTE

ALLER PLUS LOIN DANS LA SYNERGIE DES ÉQUIPES ... 31
IMPLIQUER L'ÉQUIPE : L'EXPÉRIENCE DE JEU À LA MÉDIATHĖQUE DE CONFLANS-SAINTE-HONORINE.....32 par Élise Ybled

LE JEU DE SOCIÉTÉ À LA MÉDIATHĖQUE BLAISE-CENDRARS ...32 LE JEU VIDÉo À LA MÉdIATHÈQUE BLAISE-CENDRARS 34

LE CADRE JURIDIQUE DU JEU EN BIBLIOTHÈQUE : ACQUISITIONS, CONSULTATION, PRÊT 42 par Thomas Fourmeux et Lionel Maurel COMPLEXITÉ DE LA NATURE JURIDIQUE DES JEUX

ACQUISITION DES JEUX :

LE DISTINGUO ENTRE MODÈLE

"B TO B" ЕT "B TO C" 44

CONSULTATION SUR PLACE ET PRÊT DE JEUX EN BIBLIOTHĖQUE....46 USAGES NUMÉRIQUES, TABLETTES ET APPLICATIONS 48

UN CONTEXTE JURIDIQUE FRAGILE POUR LES BIBLIOTHĖQUES

DES JEUX VIDÉo À L'INSTITUT FRANÇAIS .51

par Anthony Avila 
JEUX VIDÉO ET CULTURE FRANÇAISE

DANS LES INSTITUTS FRANÇAIS ..... 52

LA PRATIQUE DU FRANÇAIS PAR LE JEU VIDÉO : QUELQUES PISTES ....... 52

++++++++++++++++++++++++++++++++++++

ENCADRÉ :

«Et ils en pensent

quoi, les collègues?» ..p. 39

$+++++++++++++++++++++++++++++++$

\section{PARTIE II. ACQUÉRIR,} VALORISER. .55

CONSTITUER UNE COLLECTION DE JEUX : QUELS OBJECTIFS ?.......56 par Anthony Avila

LE PROJET . .56

UN FONDS DE JEUX

"CLASSIQUES " 58

UN FONDS DE JEUX VIDÉO .61

JOUER EN BIBLIOTHĖQUE, JOUETS EN BIBLIOTHĖQUE ?

par Caroline Makosza

POURQUDI DES JOUETS ? 64

QUELS AMÉNAGEMENTS POUR LES ESPACES JEUX?

JOUER SÉRIEUSEMENT - POUR APPRENDRE ? 69

par Éric Sanchez

JEU SÉRIEUX, EXÉGÈSE

D'UN TERME POLYSÉMIQUE.
JEU ET MOTIVATION .72

LE JEU, ESPACE DE RÉFLEXIVITÉ .....74

PERSPECTIVES POUR

UNE PÉDAGOGIE ALTERNATIVE .77

CRÉER UN SERIOUS GAME

EN BIBLIOTHĖQUE :

L'EXEMPLE DE ESCOUADE B .79

par Thierry Robert

LE JEU SÉRIEUX : ENTRE JEU

VIDÉO ET JEU ÉDUCATIF .80

DÉVELOPPER LES COMPÉTENCES INFORMATIONNELLES DES PUBLICS : LA BIBLIOTHĖQUE COMME ALTERNATIVE...................................... 83

LE PROJET ESCOUADE B 84

LES LUDO'MALLES DE LA MÉDIATHĖQUE DÉPARTEMENTALE DU PUY-DE-DÔME : UN OUTIL LÉGER POUR UN AUTRE REGARD SUR LES BIBLIOTHĖQUES................89

par Solenne Waszak

À L'ORIGINE DES LUDO'MALLES.......89

COMMENT PROCÉDER ? .91

LE GUIDE D'ACCOMPAGNEMENT : OUTIL DE MÉDIATION ESSENTIEL ... 92

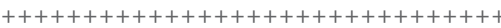
ENCADRÉS :

Le système de classification ESAR :

4 grandes catégories de jeux .p. 58 
Les 5 espaces jeux de

la ludo-médiathèque de Fosses p. 67

Ludo'malle : mode d'emploi p. 93

$+++++++++++++++++++++++++++++++$

PARTIE III.

ANIMER, CRÉER 95

POURQUDI ALLER JOUER

DANS UNE BIBLIOTHĖQUE ?........... 96

par Julien Devriendt

LES CONDITIONS DU JEU .96

L'EXPÉRIENCE DE JEU

EN BIBLIOTHĖQUE

98

LE JEU COMME OUTIL DE CRÉATION : L'UNIVERS DE TOBIE LOLNESS SUR MINECRAFT. RETOUR D'EXPÉRIENCE SUR UN TRAVAIL MENÉ AVEC DES Collégiens 102

par Véronique Amar

LE JEU COMME

OUTIL DE CRÉATION. 102

LE JEU RÉGULATEUR D'UN

PROCESSUS DE CRÉATION

COLLECTIVE 105

LES ATOUTS DU PROJET ET

SES APPORTS À COURT

ET MOYEN TERME 108

JOUER POUR JOUER .110

par Caroline Makosza
UN PROJET EN MOUVEMENT :

SIX ANS DE RÉFLEXION 110

DÉCIDER DE JOUER :

LIBERTÉ POUR LES OURSONS ! ....... 110

JOUER POUR JOUER :

AUTANT EN EMPORTE LE FLOW........112

UN PROJET DE SERVICE :

LE SENS DE L'AVIS 114

LES COLLECTIONS :

LOCALISATION, ESPACES,

TRAITEMENT, USAGES

$+++++++++++++++++++++++++++++++$

ENCADRÉ :

Concevoir un projet d'animation avec le jeu vidéo : 6 questions clés......101

$+++++++++++++++++++++++++++++++$

PARTIE IV.

PARTICIPER 121

\section{LA GAMIFICATION DE}

LA BIBLIOTHĖQUE 122

par Thierry Robert

GAMIFIER LE LIEU BIBLIOTHÈQUE. 123

GAMIFIER LES COLLECTIONS

EN BIBLIOTHÈQUE 124

GAMIFIER LES SERVICES

ET LA PARTICIPATION 125

LA GAMIFICATION : QUEL AVENIR EN BIBLIOTHĖQUE? 126 
COMMUNIQUER AUTREMENT AVEC LES ÉTUDIANTS : L'EXPÉRIENCE DU JEU EN RÉALITÉ ALTERNÉE À LA BIBLIOTHĖQUE MARIE CURIE DE L'INSA LYON................................. 128

par Guillemette Trognot

et Marie-Paule Voüta

L'ORIGINE DU PROJET 128

LE JEU EN RÉALITÉ ALTERNÉE .132

L'IMPACT DU JEU

ET SES BÉNÉFICES .136

AU-DELÀ DU JEU. 137

LA CHASSE AU TRÉSOR :

DES LAPINS INTELLIGENTS

À LA BIBLIOTHĖQUE. 140

par Florence Carre

LA BIBLIOTHĖQUE HORS LES MURS. 141

LA CHASSE AU TRÉSOR 142
SE FORMER POUR

ENTRER DANS LE JEU

147

par Julien Devriendt et Sophie Jacob

$+++++++++++++++++++++++++++++++$

ENCADRÉS :

Feuille de route des enfants .144

Offres de formation pour le jeu et/ou le jouet : quelques pistes.. .148

$++++++++++++++++++++++++++++++$

MÉMENTO

GLOSSAIRE 159

BIBLIOGRAPHIE 167

\section{INDEX DES JEUX}

ET JOUETS

169

LISTE DES AUTEURS 171 


\section{MODE D'EMPLOI par Julien Devriendt}

Dès l'apparition des premières ludothèques en France au début des années 1970, les bibliothèques ont commencé à accueillir en leur sein des espaces de jeu. Ainsi en 1975, le projet de médiathèque centrale d'HéninBeaumont ${ }^{1}$ prévoit la création d'un espace ludothèque. Ce projet s'inscrit dans la vague de création de médiathèques de cette période. Quittant leurs fonctions patrimoniales, ces établissements ont pour objectif de s'adresser à un public le plus large possible et vont dépasser le seul cadre de la culture légitime scolaire (et du livre) pour intégrer bandes dessinées, disques et vidéos. Des espaces « jeunesse » font ainsi leur apparition avec un personnel dédié, des collections et des locaux spécialement aménagés. C'est dans ce cadre que nous allons principalement retrouver les premières offres de jeu en bibliothèque.

L'offre de jeu se développe dans les années 1980-1990 y compris dans les espaces réservés aux adultes. Les médiathèques prennent conscience de leur rôle social et développent de nouvelles médiations en intégrant le divertissement et les loisirs.

Mais l'introduction du jeu en bibliothèque n'est pas chose aisée. En 2008, un article paru sur le blog professionnel de Nicolas Alarcon, membre de la bibliothèque universitaire (BU) d'Angers, présentait la mise en place d'une console Wii en accès libre au sein de la $\mathrm{BU}^{2}$. Pour annuler leur pénalité de retard, les lecteurs pouvaient défier un bibliothécaire. Bien sûr il s'agissait d'un canular qui a suscité un débat intéressant sur l'intégration de ce type de service en bibliothèque. Certains saluant l'originalité du service s'inscrivant « dans une conception élargie de la BU comme lieu de nouvelles sociabilités », d'autres défendant " la bibliothèque comme lieu unique de transmission des savoirs devant être protégé des effets de mode, du marketing ».

1. "Bibliothèque municipale d'Hénin-Beaumont (Pas-de-Calais) ", Bulletin d'informations de l'ABF, 1975. [En ligne] : < http://www.enssib.fr/bibliotheque-numerique/index-des-revues?id_ article $=54495>$.

2. «Une Wii dans ta BU », Assessment Librarian, 2008 [En ligne] : < https://assessmentlibrarian. wordpress.com/2008/03/12/une-wii-dans-ta-bu/ >. 
Récemment, de nombreux collègues se sont intéressés à la place du jeu vidéo au sein de leurs collections. Certains équipements sont même allés plus loin et n'hésitent pas à repenser plus largement la place du jeu en nouant des partenariats avec les ludothèques ou en développant de nouveaux espaces dédiés. Il ne s'agit plus de considérer uniquement le jeu comme un produit d'appel mais de montrer la diversité des jeux, leurs univers, leurs auteurs, leurs diversités. L'engouement des jeunes adultes pour les jeux de plateau marque l'importance du jeu dans nos pratiques culturelles bien au-delà du seul jeune public. Il faut ainsi repenser les espaces, le rôle des personnels et leur rapport aux usagers pour intégrer pleinement le jeu au cœur du projet d'établissement.

Lieux de sociabilité et d'accompagnement à l'éducation tout au long de la vie, ludothèques et bibliothèques partagent les mêmes valeurs d'éducation populaire, de démocratisation, d'accès à la culture. C'est sur la manière de proposer le jeu que les différences entre les deux structures vont s'opérer. La ludothèque a pour mission de " donner à jouer », mettant en avant le plaisir de jouer. S'appuyant sur la définition de Johan Hazuinga ${ }^{3}$ le jeu est une activité libre, fictive et gratuite, sans attente de résultat. À l'inverse, en l'absence d'un fonds dédié à la consultation sur place, de nombreuses bibliothèques vont utiliser le jeu comme outil d'animation ponctuel pour favoriser la lecture et l'accès à l'information.

Dans la première partie, Connaître le contexte, Céline Meneghin retrace l'évolution du jeu dans nos sociétés et de la place croissante qu'il occupe actuellement. Le jeu vidéo est la seconde industrie culturelle derrière le livre. Les jeux de société et le jouet se portent bien également avec 140 millions d'unités vendues et près de 1000 nouveautés de jeux de société par an. Preuve supplémentaire de l'engouement du public pour le jeu, l'apparition de nombreux événements, festivals, salons, autour du jeu. Autant d'occasions pour les communautés*4 de joueurs de se retrouver et de partager une passion, une culture commune. Permettre de jouer en bibliothèque devient l'occasion d'enrichir les activités des établissements

3. Johan Huizinga, Homo ludens : essai sur la fonction sociale du jeu, Paris, Gallimard, 1988 (coll. Tel ; 130).

4. Les termes suivis d'un astérisque (à leur première occurrence) sont définis dans le glossaire en fin d'ouvrage. 
en créant de nouveaux partenariats, en proposant de nouveaux services pour que le public puisse s'approprier l'équipement.

Deux contributions traitent de l'accompagnement du personnel dans la conduite de projet. Catherine Di Sciullo de la Gaîté lyrique abordera la formation continue d'une équipe dans un service déjà fortement engagé dans la médiation du jeu, puis la contribution d'Élise Ybled témoignera des différentes étapes d'intégration d'un espace de jeux à la médiathèque Blaise-Cendrars de Conflans-Sainte-Honorine. Toutes les deux, au cours d'expériences très différentes, insistent sur l'importance de cet accompagnement. En effet, le développement de nouveaux services ne peut se faire sans l'acquisition de nouvelles compétences. Il s'agit donc pour le responsable de « tracer une voie, de convaincre de faire participer, de responsabiliser, de rendre les personnels autonomes et confiants. » Un échange de questions-réponses de différents personnels de la médiathèque accompagne la contribution.

Thomas Fourmeux et Lionel Maurel nous présentent ensuite le cadre juridique lié au jeu en bibliothèque. Comme pour d'autres supports auparavant, ils soulignent l'importance de faire émerger un cadre légal afin de sécuriser ces pratiques par la mise en place d'une offre adaptée aux bibliothèques. Pour élargir les frontières, Anthony Avila s'interroge sur la création d'une offre de jeu vidéo pour promouvoir la culture française à l'étranger. Il nous fait part de son expérience auprès du réseau culturel de l'Institut français et livre des pistes d'utilisation de ces fonds comme outils pour les enseignements de français langue étrangère (FLE).

La deuxième partie de cette BA0, Acquérir, valoriser, aborde la question de l'acquisition des fonds. Quels jeux choisir ? Selon quels critères ? Il n'existe bien entendu pas de réponses définitives mais l'auteur, Anthony Avila, pose les questions pour aborder le sujet de façon globale. Un personnel qualifié sera bien sûr une des clés de la réussite de la mise en place d'un fonds de jeux.

Si l'on veut proposer une offre de jeu, il est important de ne pas mettre de côté les jouets. Caroline Makosza rappelle l'importance du jeu symbolique pour l'enfant. À travers le jouet, il va pouvoir rejouer son quotidien, expérimenter la vie en société. L'aménagement doit permettre aux enfants d'être créatifs, de détourner les objets pour mieux se les approprier. Les 
règles de fonctionnement de l'espace et son aménagement devront ainsi être pensés de manière à mettre le jeu et les usagers au centre du projet. Le jeu peut également être un levier d'apprentissage et de découverte très puissant. On pense bien sûr à l'utilisation des serious games*, où pour favoriser l'investissement des apprenants dans leur formation on va s'appuyer sur des mécanismes ludiques. Pour Éric Sanchez, « le jeu n'est pas dans la chose mais dans l'usage qu'on en fait », soulignant ainsi que l'intérêt éducatif d'un jeu dépend moins de « ses propriétés intrinsèques que de la manière dont l'enseignant ou le formateur l'intègreront dans leurs dispositifs de formation ». Des bibliothèques se sont lancées dans la création de serious games. Le réseau des bibliothèques de Montréal a ainsi mis en ligne Escouade B, jeu permettant d'apprendre à évaluer les informations sur Internet. Thierry Robert revient sur cette expérience depuis les origines du projet jusqu'à son évaluation.

Solenne Waszak de la médiathèque départementale du Puy-de-Dôme nous présente le projet des Ludo'malles. Un dispositif simple permettant aux différentes bibliothèques du territoire de tester une offre de jeu simple à prendre en main par le personnel et s'adressant à un public le plus large possible. Favorisant ainsi la convivialité et la perception de la bibliothèque comme lieu d'échange et de rencontre.

Pourquoi venir jouer en bibliothèque ? La troisième partie, Animer, créer, aborde cette question de plusieurs points de vue.

Dans le sillage de Scott Nicholson ${ }^{5}$, la contribution de Julien Devriendt s'attache à démontrer que l'important ce n'est pas le jeu, mais l'expérience de jeu*. On va venir à la bibliothèque parce qu'on nous donne accès non seulement à des jeux mais à une expérience de jeu. Cette expérience de jeu peut être différente, à la fois par le jeu lui-même en proposant une sélection de jeux originaux et peu connus du grand public ou en proposant une manière différente de jouer à un jeu que le public possède déjà.

Comme on l'a vu plus haut avec Éric Sanchez, l'important n'est pas le jeu mais ce que vous allez en faire. Il est ainsi possible de détourner des jeux à des fins de création. Véronique Amar présente un retour d'expérience

5. Scott Nicholson, Everyone Plays at the Library: Creating Great Gaming Experiences for All Ages, Medford NJ, Information Today, 2010. 
sur l'atelier Minecraft mené par la Bibliothèque municipale de Lyon, en partenariat avec l'équipe pédagogique d'un collège et leurs élèves, autour de l'univers du roman Tobie Lolness.

Permettre l'expérience du jeu, inventer d'autres utilisations du jeu, mais à quelles fins ? Dans sa contribution, Caroline Makosza, responsable d'une ludo-médiathèque (Fosses) insiste sur la notion de libre jeu. Le jeu cesse d'être jeu dès lors qu'il est instrumentalisé. Il est nécessaire de s'éloigner de la vision utilitariste du jeu comme produit d'appel. Le jeu doit être présenté pour lui-même, sans chercher à savoir quels apprentissages concrets l'enfant va en retirer. "Le jeu n'est éducatif que s'il ne cherche pas à l'être ${ }^{6}$. Le rôle du professionnel sera de mettre à disposition un espace, d'organiser des collections, des services et de laisser faire. Mais cela implique également beaucoup d'observations et de réaménagements pour correspondre le plus possible aux usages du public.

Une quatrième partie, Participer, rend compte du développement de la participation ${ }^{7}$ dans les bibliothèques, au travers de trois projets menés auprès d'adolescents, d'étudiants et d'enfants.

Le jeu ne se limite plus à la table de jeu ou à l'écran mais investit l'univers physique. La gamification* (ou ludification)* vise à utiliser notre propension naturelle à jouer pour améliorer la participation des " joueurs » en transposant des mécaniques de jeu* dans un domaine non-ludique. Thierry Robert présente le jeu Find the future menée à la bibliothèque publique de New York au cours duquel les joueurs pouvaient explorer la bibliothèque à la recherche de ses documents les plus précieux.

Guillemette Trognot et Marie-Paule Voïta reviennent sur la conception, l'organisation et la mise en œuvre d'un jeu en réalité alternée* et son impact à la bibliothèque de l'Insa Lyon. Cette expérimentation combinait l'utilisation des réseaux sociaux et l'organisation d'événements sur le campus pour prolonger l'expérience des joueurs en allant les chercher où ils se trouvent. C'est une démarche similaire, utiliser la bibliothèque et

6. Martine Mauriras-Bousquet, « Jouer et apprendre : même combat, le rôle des ludothèques dans la promotion du libre jeu ", Actes des universités d'été des ludothécaires, Paris, Association des ludothèques françaises, 1998, pp. 125-128.

7. Note de l'éditeur : voir, pour la participation, l'ouvrage Construire des pratiques participatives dans les bibliothèques, sous la direction de Raphaëlle Bats, Villeurbanne, Presses de l'enssib, 2015 (coll. La Boîte à outils ; 33). 
ses ressources comme terrain de jeu pour mieux se l'approprier, qui est à l'origine du projet mené par la médiathèque des Ulis. Florence Carre nous présente un projet ambitieux de chasse au trésor mêlant objets numériques et documents traditionnels.

D’une manière générale, jouer et faire jouer en bibliothèque participe d'une conduite de changement qui s'accompagne et s'expérimente, sans solutions toutes faites ou recettes transposables. Pour mûrir une réflexion professionnelle et faire évoluer ses pratiques, un certain bagage de compétences est nécessaire et c'est l'objet de la dernière contribution du livre : Se former pour entrer dans le jeu ; Julien Devriendt et Sophie Jacob recensent différents organismes de formation et lieux ressource, autant de pistes pour construire et enrichir peu à peu les métiers des professionnels des bibliothèques. 


\section{PARTIE I}

\section{CONNAÎTRE LE CONTEXTE}

1. LE JEU COMME BIEN CULTUREL ET COMMUN par Céline Meneghin

$++++++++++++++++++++++++++++++++++++++++++++++++++++++++++++++++$

2. ACCOMPAGNER LES ÉQUIPES

LA MÉDIATION NUMÉRIQUE : UN PROJET D'ÉQUIPE À LA GAÎTÉ LYRIQUE

par Catherine Di Sciullo

IMPLIQUER L'ÉQUIPE : L'EXPÉRIENCE DE JEU À LA MÉDIATHÈQUE DE CONFLANS-SAINTE-HONORINE par Élise Ybled

3. LE CADRE JURIDIQUE DU JEU EN BIBLIOTHĖQUE : ACQUISITIONS, CONSULTATION, PRÊT par Thierry Fourmeux et Lionel Maurel

$++++++++++++++++++++++++++++++++++++++++++++++++++++++++++++++++$

4. DES JEUX VIDÉo À L'INSTITUT FRANÇAIS

par Anthony Avila 


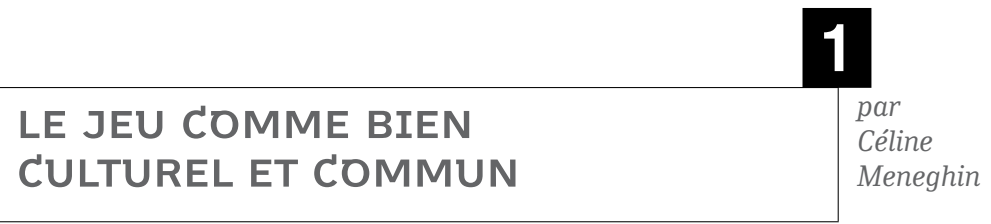

\section{JEUX DE SOCIÉTÉ, JEUX ET SOCIÉTÉS}

L'histoire du jeu débute avec l'histoire de l'humanité. Tout au moins celle qui nous est connue par les vestiges archéologiques et les trop rares sources qui nous sont parvenues. Depuis la plus haute Antiquité, l'être humain joue. Les civilisations mésopotamiennes et égyptiennes notamment nous ont transmis des jeux de dés, d'osselets, de courses ou de cases, comme le Senet. On trouve également de nombreux jeux en Chine ou en Inde.

Un constat s'impose vite à qui s'intéresse à l'histoire des civilisations : le jeu est présent dans toutes les sociétés de par le monde. Partout il revêtait, et revêt encore en grande partie, une aura mystique, symbolique liée au hasard induit dans chaque jeu. D'abord réservés à l'élite sociale - rois, prêtres, guerriers - les jeux se sont ensuite popularisés, ont voyagé avec les hommes, ont été transmis oralement puis par écrit, ont été adoptés et adaptés par les différentes sociétés ${ }^{1}$. Le jeu de tout temps a permis les échanges entre les hommes de langue et de culture différentes.

\section{UN MIROIR DES ÉVOLUTIONS TECHNIQUES}

Le jeu a évolué en même temps qu'il a été adapté aux mentalités et aux techniques. Ainsi, les règles transmises oralement pendant des siècles et à travers les aires géographiques, commencent à être fixées par écrit en Occident avec l'invention de l'imprimerie. Cette transmission orale ne s'est néanmoins pas totalement perdue. Les joueurs continuent à apprendre les règles d'autres joueurs qui les transmettent à leur tour.

L'évolution des techniques d'impression et de création du papier a permis l'apparition de jeux nouveaux. Les jeux de cartes apparaissent en

1. On peut citer les multiples variantes du jeu de semailles sur le continent africain ou le Weiqi, jeu chinois très ancien, importé au Japon au vil siècle, époque à laquelle la culture chinoise est adoptée par la cour impériale japonaise, qui est alors devenu le jeu de Go. 
Chine au IX ${ }^{\mathrm{e}}$ siècle à l'époque où la technique de fabrication du papier évolue du rouleau à la feuille. En Occident, les cartes à jouer font leur apparition au milieu du XIV ${ }^{\mathrm{e}}$ siècle et connaissent une forte expansion avec l'imprimerie et l'évolution des techniques de xylographie. Le siècle des Lumières a été particulièrement friand de jeux. On assiste à un essor et une diversification spectaculaire des supports et des règles : jeux de loterie, de plateau, de mot et d'esprit. Les maisons de jeux se développent. Les techniques d'ébénisterie et de marqueterie contribuent à la création de tables de jeux variées et inventives.

La société au XIX ${ }^{\mathrm{e}}$ siècle a vu la multiplication des jeux et des jouets grâce à l'industrialisation et la mécanisation. L’Allemagne, en particulier, devient entre 1850 et 1950, la première productrice mondiale de jeux. Avant la première guerre mondiale, ce pays produit à lui seul la moitié des jeux mondiaux avant que ne se développent les industries américaines, anglaises et japonaises. De grosses sociétés spécialisées dans l'édition de jeux voient le jour comme Ravensburger ou Nintendo. À cette époque, le jeu commence à être théorisé, à être utilisé à des fins éducatives, pédagogiques, religieuses auprès des enfants. Les activités parents et enfants sont promues et appréciées des familles bourgeoises pour toutes ces raisons. L'utilisation du jeu à des fins de propagande auprès des enfants connaît son apogée durant la Grande Guerre dans toutes les nations belligérantes. Il perd de son caractère mystique et même ludique, mais conserve son côté social. Au xx ${ }^{\mathrm{e}}$ siècle, l'adaptation aux techniques se poursuit. Dans les années 1950, des normes sont élaborées, le jeu devient un produit standardisé, de masse et surtout il doit être bon marché pour se diffuser dans les familles. Il apparaît également sur les nouveaux médias : radio, télévision, Internet. Il devient jeu vidéo avec le développement de l'informatique. Cette grande plasticité technique des jeux et leur permanence dans les sociétés au fil des époques montrent qu'ils témoignent d'une activité essentielle.

\section{UNE ACTIVITÉ SOCIALE}

Très tôt, l'enfant joue pour appréhender le monde, en apprendre les codes sociaux et le langage. Par les jeux symboliques, très présents aux premiers 
âges, il apprend à reproduire des comportements, il développe son imaginaire et avance dans la construction de soi.

Le jeu est une pratique naturelle à tout âge, importante dans la vie quotidienne des êtres humains et dans leur développement collectif et personnel. L'enfant comme l'adulte recherche le jeu et ses codes. En 1958, Roger Caillois $^{2}$ théorisait ainsi le jeu autour de 6 principes :

\section{Les 6 principes du jeu, selon Roger caillois}

Le jeu est une activité :

- libre : si elle devient contrainte, ce n'est plus du jeu ;

- séparée : avec ses temps et lieux propres ;

- incertaine : le hasard y tient une place importante, l'issue n'est pas connue à l'avance, sinon cela tue l'esprit du jeu ;

- improductive : un divertissement, sans aucun autre but ;

- réglée : elle possède ses règles propres ;

- fictive : détachée de la vie réelle, se déroulant dans des univers simulés.

C'est donc pour la liberté que permet le jeu, l'imaginaire qu'il développe, en dehors de toute hiérarchie sociale, et le plaisir de l'activité, que l'on joue. Le jeu apporte aussi beaucoup sur le plan émotionnel et relationnel. Il est grisant de jouer, on ressent beaucoup d'émotions, de la joie de la victoire à la frustration de la défaite en passant par l'euphorie du suspense. On apprend beaucoup sur soi et les autres en jouant. Par les différentes postures des joueurs, le " bon ou le mauvais joueur ", par la confrontation à ses propres limites, l'humain poursuit son développement tout au long de la vie. Il se contraint volontiers aux rituels des jeux : apprentissage des règles, transmission de ces règles*, installation du jeu*, respect des règles pour éprouver ce plaisir de jouer. Il recherche le lien social et intergénérationnel car chaque partie, chaque rencontre l'enrichit, il éprouve le plaisir d'être ensemble, de tisser des liens, ne serait-ce que le temps d'une partie. Dans notre société des loisirs où l'épanouissement personnel a pris une place prépondérante, cela est d'autant plus primordial.

2. Roger Caillois, Les jeux et les hommes : le masque et le vertige, 8e éd., Paris, Gallimard, 1958. 


\section{CULTURE dU JeU DANS NOS SOCiÉtÉs ACtuelLeS}

L'industrialisation et la massification de la production de jeux, ainsi qu'un temps plus important disponible et consacré aux loisirs, ont permis le développement de la pratique ludique et d'une culture autour du jeu.

\section{Le marché du jeu et du jouet en France}

Preuve de l'engouement des Français pour le jeu, ce dernier connaît (jeux et jouets confondus) un chiffre d'affaires annuel autour de 3 millions d'euros, ce qui représente près de 140 millions d'unités, dont 12 millions de boîtes de jeux de société. Chaque ménage dépense 200 euros par an et par enfant. Près de 1000 nouveautés sortent chaque année pour ce qui concerne le jeu de société3. Même si le marché connaît des mutations avec l'apparition des tablettes tactiles pour enfant rencontrant un fort succès conjoncturel, le jeu traditionnel, le jeu de société et les jouets restent des valeurs sûres. Pour ce qui est du jeu vidéo, les chiffres sont faramineux : le Syndicat des éditeurs de logiciels de loisirs (SELL) ${ }^{4}$ indique qu'il représente un chiffre d'affaires de 2,7 milliards d'euros ${ }^{5}$, ce qui en fait la deuxième industrie de loisirs en France après le livre. Quel que soit le support, les Français jouent, beaucoup. Ils sont les deuxièmes à consacrer le plus de temps et de budget aux pratiques ludiques après les Anglais et devant les Allemands en Europe.

\section{une industrie culturelle classique}

Le monde du jeu fonctionne comme les autres industries culturelles : auteurs, éditeurs, diffuseurs sont autant d'acteurs de la chaîne du jeu. Les créateurs de jeux* sont appelés " auteurs ». Leur travail de création repose sur un univers permettant l'imaginaire, ils doivent élaborer des règles plaisantes, créatives et des mécaniques de jeu qui captivent les joueurs. L'émergence des auteurs et leur reconnaissance sont un phénomène relativement récent que ce soit pour le jeu ou pour le jeu vidéo.

\footnotetext{
3. « Jeux et jouets : l'étude complète du secteur », Pépites ludiques, 2012. [En ligne] : < http://www. pepitesludiques.com/article-jeux-et-jouets-l-etude-complete-du-secteur-113808872.html >.

4. < http://www.sell.fr/ >

5. < http://www.essentiel-jeu-video.fr/media/pdf/EJV_02_FR.pdf >.
} 
Avant les années 1960, il était rare de pouvoir les identifier ${ }^{6}$. Désormais, les auteurs sont connus, leur nom apparaît sur les boîtes des jeux. Les auteurs reconnus sont devenus des gages de qualité pour les jeux qui portent leur nom. Parmi ceux-ci ${ }^{7}$, on peut citer l'Allemand Reiner Knizia, auteur de plusieurs dizaines de jeux de tous types, du jeu de stratégie réservé aux joueurs expérimentés comme Res Publica, au jeu de cartes familial comme Schotten-Totten, les Français Bruno Faidutti, auteur entre autres du jeu Citadelles et Philippe des Pallières, auteur de La Guerre des moutons et de Les Loups-garous de Thiercelieux. La créativité des auteurs est fortement encouragée en France par plusieurs concours de création* proposant aux jeunes auteurs de venir présenter leurs prototypes de jeu au public et à un jury de spécialistes, à l'exemple du Concours international de créateurs de jeux de société de Boulogne Billancourt organisé par le Centre national du jeu ${ }^{8}$ qui existe depuis 1977. Ces concours permettent de donner une visibilité à de jeunes talents auprès d'éditeurs potentiels. Les illustrateurs tiennent également une place importante dans le processus de conception d'un jeu. Un jeu original peut passer à côté de son public si ses illustrations ne sont pas suffisamment attractives. Tout comme les auteurs, les illustrateurs sont également crédités sur les boîtes des jeux et certains sont désormais reconnus internationalement comme François Bruel ou Julien Delval. Le rôle de l'éditeur enfin, est similaire à celui de l'industrie du livre, de la sélection à la diffusion, en passant par la mâ̂trise des normes en vigueur. Il diffuse le plus souvent lui-même ses productions. La France a la particularité de compter un réseau important de boutiques spécialisées, environ 400, où l'on trouve tous les types de jeux.

6. On peut noter l'exception de John Jefferys créateur du jeu A Journey through Europe, publié par Ravensburger en 1759, qui a connu un succès tel qu'il fut traduit dans de multiples langues avec des déclinaisons dans de nombreux pays (France, Suisse, Chine, etc.) encore éditées de nos jours. Mais aussi celle de Charles B. Darrow qui créa dans les années 1930 le Monopoly® à partir de l'idée d'Elizabeth Magie.

7. Voir la rubrique « Répertoire » du site : < www.trictrac.net $>$.

8. < http://www.centrenationaldujeu.com/ >. 


\section{La « culture jeu » : festivals, salons, associations...}

Ce dynamisme du secteur du jeu se retrouve dans la mobilisation du public pour les événements grand public de plus en plus nombreux. Parmi ces événements, deux en particulier sont majeurs en France. Il s'agit du Festival international des jeux de Cannes, créé en 1987 et du Festival ludique international de Parthenay (FLIP), créé en 1986. Le festival cannois fait référence dans le monde francophone du jeu et décerne L'As d'or du jeu de l'année, récompensant le meilleur jeu de société édité dans l'année. Le festival de Parthenay met l'accent sur la création dans le cadre d'un concours qui remet les FLIP Créateurs. Le succès est au rendez-vous à chacune des éditions de ces deux festivals fréquentés par 130000 à 175000 personnes. De nombreuses villes leur ont emboîté le pas. La plupart des grandes et moyennes villes françaises proposent un salon du jeu : Paris, Toulouse, Pau, Montpellier, Rouen, Lille, Issy-les-Moulineaux et bien d'autres encore.

Si ces événements plaisent tant au public, c'est qu'ils permettent de jouer à tous les types de jeux : de rôle*, de plateau, vidéo, de cartes, historique ou de figurine. Le public peut également y découvrir les dernières nouveautés, jouer aux jeux primés par le Spiel des Jahres ${ }^{9}$ ou l'As d'or, rencontrer les auteurs, les illustrateurs et les éditeurs, tester des prototypes, participer à des tournois ou simplement se mettre autour d'une table avec de parfaits inconnus le temps d'une partie libre. Très souvent, les univers des jeux s'inspirent d'autres genres : fantasy, science-fiction, bande dessinée ou encore manga. Les festivals de jeux sont l'illustration parfaite de ce brassage des cultures populaires. On y rencontre des gens de tous les âges, des familles, des communautés de joueurs, des cosplayers*, le tout dans une ambiance très conviviale.

\section{Les communautés de joueurs}

Les communautés de joueurs sont également très actives sur Internet. Il existe de nombreux forums et groupes sur les réseaux sociaux consacrés aux différents types de jeux, sur lesquels les joueurs échangent sur leurs

9. Le Spiel des Jahres a été créé en 1976. Il est le prix de référence mondiale dans le domaine du jeu. 
expériences de jeux, postent des critiques, organisent des événements et des parties. Le site francophone de référence, Tric Trac ${ }^{10}$ est une véritable mine d'or pour qui s'intéresse au jeu et à son actualité. Il recense plus de 150000 jeux et est fort d'une communauté de plus de 23600 joueurs. Au-delà d'une communauté sur Internet, le but des joueurs est de se rencontrer autour de jeux qu'ils souhaitent partager. Pour cela, de nombreuses associations et clubs de jeux existent partout en France, ainsi qu'un réseau national de ludothèques. Des musées sont consacrés aux jeux et à leur histoire, comme le musée du jouet à Poissy ou celui de Moirans, d'autres les intègrent dans leurs collections, comme l'Historial de la Grande Guerre ${ }^{11}$. Ils développent des propositions d'activités et d'ateliers pour le public. Autant de pistes à explorer et de partenaires potentiels pour les bibliothèques.

\section{JOUER EN BIBLIOTHĖQUE}

Les missions des bibliothèques sont d'apporter des contenus à leurs usagers, qu'ils soient informatifs ou de divertissement. Le jeu, à la fois contenu culturel et pratique populaire, s'adresse à toutes les générations et classes sociales. Il est plébiscité par un public diversifié de tous âges, lecteurs et non-lecteurs. Il trouve donc à ce titre sa place parmi les différentes offres qu'une bibliothèque doit offrir à la population. Les bibliothèques se sont toujours adaptées aux nouveaux supports, aux nouvelles pratiques culturelles. Le jeu est l'un des moyens de s'adresser à un public qui ne les fréquente pas ou plus, de même que la musique ou la vidéo ont pu le faire.

Dans certains départements, hormis dans la ville chef-lieu, aucune offre de jeu n'est disponible pour la population. Proposer du jeu en bibliothèque est également important dans les zones rurales où les ludothèques sont peu présentes, et s'adressent principalement aux enfants. La bibliothèque est souvent dans ces villages le seul service de proximité permettant la convivialité, la rencontre entre les habitants, le maintien du lien social.

10. $<$ www.trictrac.net $>$.

11. < http://www.historial.org/Musee-collection/Collection/Collections-thematiques/Les-jeux-etjouets-de-1914-1918 >. 
Le jeu permet également la mise en valeur des savoir-faire des bibliothèques en termes d'animation et de médiation, sa capacité à créer une communauté d'usagers, à s'adresser à tous les publics et générations et à les faire se rencontrer, ses autres services et ses collections variées qui peuvent s'enrichir mutuellement, sa capacité à sélectionner et faire découvrir des ressources.

\section{Du livre au jeu et du livre-jeu*}

Le livre est loin d'être étranger au jeu et inversement. Beaucoup de jeux se basent sur les mots et les jeux de mots, sur la construction de trames narratives ou sur des associations d'idées. Parmi ces jeux, les jeux de rôle bien sûr, mais aussi des jeux de plateau ou de cartes faisant intervenir l'inventivité et l'imagination des joueurs autour des histoires et des mots, comme Dixit, Il était une fois, Linq ou Speech ${ }^{12}$. Les livres aussi peuvent se faire jeux. La collection Un livre dont vous êtes le héros des éditions Gallimard a connu un succès important auprès des adolescents dans les années 1990 et est rééditée de nos jours. Cette collection plaisait notamment pour son aspect interactif utilisant des schémas narratifs proches des jeux de rôle. L'éditeur Djeco fait très régulièrement appel à des illustrateurs jeunesse pour le design de ses jeux et objets : Benjamin Chaud, Élodie Nouhen, Aurélia Fronty pour ne citer qu'eux. Pour les plus jeunes, l'éditeur Memo ${ }^{13}$ propose une collection de livres en jeu autour des œuvres d'Anne Bertier, Anne Crausaz ou Malika Doray. Ces livresjeux permettent la découverte de l'univers d'un auteur, de ses albums en s'amusant. Ils peuvent facilement être utilisés en bibliothèque pour des animations auprès des tout-petits. Le jeu peut être un moyen d'amener la lecture plaisir, mais cela ne doit pas être une fin en soi ni le seul usage pour le jeu qui reste avant tout un divertissement, un moment de détente.

12. Chacun de ces jeux dispose d'une fiche descriptive détaillée sur le site Tric Trac.

13. < http://www.editions-memo.fr/-livres-en-jeu- >. 


\section{Créer de nouvelles opportunités}

De nombreuses bibliothèques proposent des jeux, des espaces dédiés et des animations autour de ce support et de cette culture. Preuve de l'intérêt des personnels pour cette thématique, ces dernières années ont vu la multiplication de journées professionnelles ${ }^{14}$. Les bibliothécaires échangent en ligne autour de leurs expériences, de leurs actions, s'entraident et se conseillent en plus de diffuser leurs informations. Il existe sur Facebook deux groupes professionnels, l'un consacré au jeu en bibliothèque ${ }^{15}$ et l'autre au jeu vidéo ${ }^{16}$. Le forum Agorabib ${ }^{17}$ accueille régulièrement des questions relatives au jeu.

Développer ce domaine est une opportunité pour la bibliothèque de nouer des partenariats. Les ludothèques viennent au premier plan. Leur expertise et leur professionnalisme sont des atouts précieux pour animer ensemble des séances de jeux ou construire des coopérations plus ambitieuses comme l'organisation de salons ou la participation active de la bibliothèque à la Semaine du jeu. Cela est d'autant plus facile lorsque bibliothèque et ludothèque cohabitent dans un même bâtiment, voire un même espace. La mise en place d'actions autour du jeu est également l'occasion de nouer des liens avec les associations et clubs de jeux ou encore les boutiques spécialisées qui pourront aider à l'organisation et l'animation des parties, mettre à disposition des jeux, amener un public de joueurs, aider à la diffusion de la communication et conseiller pour la constitution de la collection. Il ne faut bien sûr pas oublier les usagers eux-mêmes qui souhaitent s'impliquer, amener leurs jeux et les faire découvrir aux autres. Le jeu est tout simplement une opportunité supplémentaire d'enrichir humainement la bibliothèque.

\footnotetext{
14. Le centre national de formation aux métiers du jeu et du jouet (FM2J) propose notamment chaque année une formation intitulée « Jeu et bibliothèque ». Les bibliothèques départementales proposent également dans leurs programmes annuels ces formations en lien avec les collections qu'elles mettent à disposition, par exemple, la bibliothèque départementale de la Somme propose mensuellement des matinées ludiques à l'intention des professionnels des bibliothèques pour qu'ils s'approprient et puissent à leur tour proposer ce support.

15. Groupe Facebook Jeu en bibliothèque : < https://www.facebook.com/groups/jeubibliotheque/ >.

16. < https://www.facebook.com/groups/jvbib/ >.

17. < http://www.agorabib.fr/ >.
} 


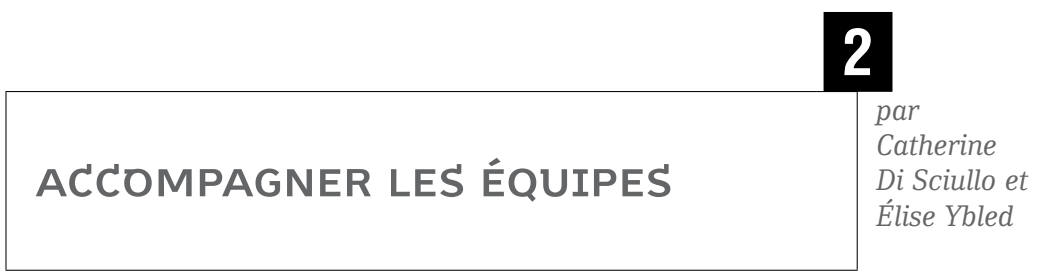

LA MÉDIATION NUMÉRIQUE : UN PROJET D'ÉQUIPE À LA GAÎTÉ LYRIQUE par Catherine Di Sciullo

$+++++++++++++++++++++++++++++++++++++++++++++++++++++++++++++++$ IMPLIQUER L'ÉQUIPE : L'EXPÉRIENCE DE JEU À LA MÉDIATHĖQUE DE CONFLANS-SAINTE-HONORINE par Élise Ybled 
LA MÉDIATION NUMÉRIQUE :

UN PROJET D'ÉQUIPE À LA GAÎTÉ LYRIQUE

par Catherine

Di Sciullo

\section{LA COMPLÉMENTARITÉ, UNE CLÉ DE RÉUSSITE}

La Gaîté lyrique, lieu culturel parisien dédié aux nouvelles formes de culture à l'ère du numérique, se dote dès son ouverture en 2011 d'un centre de ressources (CDR). Espace de travail et de découverte en accès libre, le CDR propose d'appréhender les cultures numériques et les nouvelles formes de création. Feuilleter un magazine, consulter un livre, découvrir des applications sur tablette, télécharger un ebook, écouter un vinyle ou regarder une vidéo, suivre un atelier, s'exercer à la composition musicale, des façons différentes de décrypter les cultures émergentes et les bouleversements des sociétés. Un des axes forts de la politique documentaire est l'éditorialisation. Concept affirmé partout ailleurs dans le bâtiment, il prend au CDR toute sa signification : ouvrages, applications, magazines, fanzines, vinyles, vidéos, œuvres d'artistes sont proposés aux publics du CDR afin de prolonger les visites des expositions et d'approfondir les thématiques abordées par les différents événements de la Gaîté lyrique. Ateliers, visites, expositions, restitutions d'ateliers, débats, lancements d'ouvrages ou d'applications sont autant de possibilités de rencontres, d'échanges et d'expérimentations.

L'équipe est constituée de trois documentalistes et d'une responsable. Le CDR étant ouvert 6 jours sur 7 de 14 h à 20 h, cela implique de nombreuses heures dédiées à l'accueil des publics, samedi et dimanche compris. Comment alors concilier travail documentaire (back office) et travail d'accueil et d'accompagnement des usagers d'une part et médiation numérique lors des ateliers ouverts à tous, du jeune enfant au sénior en passant par les primo-arrivants ou encore les étudiants et les professionnels, d'autre part ? La complémentarité au sein de l'équipe est essentielle et constitue la base sur laquelle s'appuie tout le travail réalisé, travail qui évolue à une vitesse vertigineuse puisqu'il s'agit de suivre les usages numériques d'aujourd'hui et d'anticiper ceux de demain. Cela implique pour le CDR d'être à la pointe en proposant toujours de nouveaux services numériques. 


\section{IDENTIFIER LES SAVOIR-FAIRE NÉCESSAIRES}

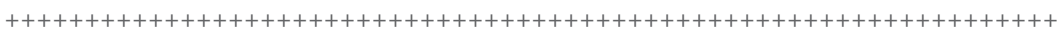

Petite équipe dit complémentarité. Il convient alors de s'assurer que les savoir-faire dont on a besoin pour fonctionner sont bien là :

- compétences informatiques ;

- compétences en audiovisuel ;

- connaissances en musique et connaissance du monde du jeu ;

- goût pour la technique, la maintenance, l'installation de dispositifs électroniques ;

- notions de gestion pour gérer les abonnements, les commandes, les droits d'auteur et de diffusion, les achats de création numériques ;

- pour un lieu international comme la Gaîté, possibilité de parler dans l'équipe plusieurs langues et de rédiger parfaitement les résumés en anglais ;

- compétences en programmation ;

- possibilité de travailler sur des logiciels différents, du logiciel de montage audiovisuel à celui de représentation en 3D pour les scénographies du CDR ;

- connaissances multiples de différents champs artistiques, dans l'équipe certains sont spécialisés en littérature numérique, en histoire de l'art, philosophie, cinéma, musique ... ;

- appétit pour concevoir et animer des ateliers tablettes auprès de divers publics en les thématisant et en s'appuyant sur les possibilités qu'offre chacune des applications. 
Bien d'autres compétences apparaissent et se révèlent au cours de la création de nouveaux services, de mises en place de nouveaux espaces ou lors de nouvelles collaborations avec un partenaire ou un artiste. Si le recrutement initial d'une équipe consolide un pôle de créativité, l'évolution que l'on imprime à un service créé, fait émerger, développer des nouvelles compétences pour chaque personne.

\section{L'AUTOFORMATION : UN DYNAMISME À ENCLENCHER}

Parce que la Gaîté lyrique se veut au cœur des nouveaux usages du numérique, les équipes font une veille assidue sur les formes émergentes de création et de communication et se font fi de proposer au CDR la possibilité d'anticiper demain, voire de se projeter dans le futur en testant des prototypes innovants ou en apprenant à utiliser de nouveaux outils. Les start-up de notre pépinière d'entreprises Créatis nous y forcent, les artistes programmés nous font découvrir leur vision du monde, la veille sur Internet et sur papier nous tient aux aguets. Mais comment faire fonctionner un centre de ressources à la pointe de l'innovation, en le pensant comme un laboratoire, un lieu d'expériences, de co-working, sans se former sans cesse ?

Ici comme ailleurs le temps et les finances manquent et le recrutement fait défaut. Sans acquisition de nouvelles compétences, pas de nouveaux services ! Il s'agit alors de réfléchir ensemble à un mode de fonctionnement qui fasse la part belle aux désirs de chacun pour impulser de l'énergie. Ainsi, les ateliers menés au CDR sont-ils conçus et animés par l'équipe de documentalistes. Les ateliers tablettes par exemple, appelés «Appli'quons-nous! » ont été possibles grâce à la mobilisation et à l'auto formation de deux documentalistes. Pas à pas, elles ont appris à connaître le monde de la création numérique indépendante, ont établi des relations de confiance avec les éditeurs et créateurs de livres interactifs ou d'applications créatives, ont construit un référentiel pour évaluer les applications les plus belles, les plus riches, les plus aptes à développer l'imaginaire chez les jeunes enfants et à fabriquer des objets numériques interactifs. L'accompagnement du responsable est primordial dans ce long cheminement. Il s'agit pour lui, me semble-t-il, de tracer une voie, de convaincre, 
de faire participer, de responsabiliser, de rendre les personnels autonomes et confiants.

\section{LA MISE EN PLACE D'ATELIERS DE DÉCOUVERTE D'APPLICATIONS SUR TABLETTE}

Cela est parti d'un premier constat : la mise à disposition des iPad avec un choix d'applications en lien avec notre programmation n'était pas satisfaisante. Même dans un lieu comme la Gaîté, le fait de demander un iPad pour consulter l'offre d'applications ne va pas de soi. Que vais-je trouver sur la tablette ? Que vais-je en faire ? Vais-je savoir me débrouiller dans la sélection? Ai-je assez de temps pour cela ? Le deuxième constat est très pragmatique : tous les parents ayant testé la tablette avec leurs enfants ont tous livré leur étonnement de trouver de si belles et si créatives applications. Très vite se pose la question de l'accès à la création numérique de qualité et aux outils critiques sur l'édition numérique jeunesse. L'intérêt des parents et éducateurs est toujours vivant face à la publication pléthorique de jeux tous très inégaux. Seule la médiation, notamment en bibliothèque, peut apporter des réponses, aiguiser le regard, aider à se forger ses propres critères d'évaluation, faire prendre conscience qu'il peut y avoir un rapport plus créatif aux écrans des tablettes et que des applications qualitatives peuvent permettre à l'enfant et à l'adulte de changer ses perceptions du monde.

Deux axes ont été suivis : des ateliers où « le faire " est prépondérant et des rencontres publiques avec les créateurs d'applications.

\section{LES ATELIERS « APPLAB »}

Les documentalistes ont dû apprendre à manier les tablettes, à sélectionner les meilleures applications selon les thèmes de chaque grande exposition transdisciplinaire, gérer la partie technique du parc de tablettes, réfléchir à un dispositif scénique (nous avons confié au designer Éric Benqué le soin de fabriquer un «Applab»), acquérir des connaissances pointues en matière d'édition numérique en France et à l'étranger, appréhender le potentiel créatif de chaque application pour pouvoir concevoir 
ex nihilo un atelier thématique à partir de 3 ou 4 applications, s'adapter à chaque groupe de public (écoliers dans le cadre des aménagements des rythmes éducatifs [ARE], familles le mercredi et le week-end, étudiants, collégiens, lycéens sur demande de l'enseignant, primo-arrivants dans le cadre de partenariat avec des associations notamment les foyers CroixRouge et Bibliothèques sans frontières, enseignants, écoliers dans le cadre des cycles « Enfance et cinéma », centres aérés, bibliothécaires ...), élaborer une politique d'indexation documentaire des applications (nous avons 250 applications indexées dans notre catalogue en ligne) ${ }^{1}$ et créer nos propres pages web pour annoncer le contenu de chaque atelier ${ }^{2}$.

Cette mobilisation de l'équipe a permis de mener 200 ateliers en un an et demi. Tous ont été proposés gratuitement et sans inscription. Le « Applab » fonctionne à plein chaque jour. Des dizaines d'enfants et parents, adolescents ou jeunes adultes viennent spontanément jouer et découvrir semaine après semaine les sélections mises en avant. Des enfants du quartier ne ratent aucun rendez-vous du mercredi et reviennent par la suite pour continuer leur expérience hors atelier, accompagnés de leurs frères ou sœurs ou copains.

Aujourd'hui, les documentalistes sont sollicitées pour animer des ateliers tablettes hors les murs. En octobre 2015, Élise Schweisguth et Laura Cattabianchi ont animé un atelier pour des professionnels et des chercheurs dans le cadre du festival Imaginascience à Annecy.

\section{DES RENCONTRES AVEC DES CRÉATEURS D'APPLICATIONS}

12 créateurs d'applications sont venus animer eux-mêmes un atelier. Ces rencontres ${ }^{3}$ permettent aux participants de questionner les professionnels de la création numérique sur outils nomades à propos de leur métier, leur parcours, leur vision de l'économie numérique, leur organisation de travail... Les enfants surtout prennent conscience qu'il y a de " vraies » personnes derrière chaque jeu. Le rapport réel/virtuel prend tout son sens.

1. < http://catalogue.gaite-lyrique.net:8080/ >.

2. Par exemple l'atelier Game Older : < http://gaite-lyrique.net/atelier/game-older >.

3. < http://gaite-lyrique.net/archives/rencontre >. 
En juin 2015, c'est Étienne Mineur, co-fondateur des Éditions Volumique, qui est venu animer un «Appli'quons-nous ! ».

\section{ALLER PLUS LOIN DANS LA SYNERGIE DES ÉQUIPES}

Fort de notre expérience au CDR, un nouveau projet pour la rentrée 2015 a été initié : la fusion du centre avec l'espace jeux vidéo voisin. Cette impulsion est donnée dans le but d'offrir un meilleur accueil des publics et une plus grande fluidité des espaces sur le premier étage, lieu totalement en libre accès et gratuit.

L'équipe du centre de ressources et celle de l'espace jeux vidéo seront amenées à travailler ensemble. Une seule banque d'accueil est prévue rassemblant le personnel des deux entités. La programmation sera commune, permettant une plus grande richesse dans l'offre de services et réitérant ainsi le fait que le jeu vidéo est une ressource à part entière au même titre qu'une œuvre culturelle. Des réunions ont déjà lieu pour imaginer le fonctionnement de demain, la scénographie des espaces, la cohésion de l'offre, l'organisation des ateliers, le rythme des médiations. L'équipe des relations aux publics, l'équipe de communication, le comité éditorial, l'équipe d'accueil et de billetterie, la boutique, l'équipe du Plateau média participent à la réflexion collégiale.

Un nouveau challenge pour les équipes ! 
IMPLIQUER L'ÉQUIPE :

L'EXPÉRIENCE DE JEU À LA MÉDIATHĖQUE

DE CONFLANS-SAINTE-HONORINE

Le jeu a trouvé sa place à la médiathèque Blaise-Cendrars (Yvelines). Qu'il soit de société ou vidéo : on joue! Son introduction puis sa présence ont généré bien des inquiétudes au sein des équipes, liées au bouleversement organisationnel induit par un nouveau fonds et donc de nouveaux usages. Comment sont nés ces projets ? Comment impliquer les équipes dans le processus de création et de mise en place du projet de jeu (qu'il soit de vidéo ou de société) afin qu'elles en soient moteur pour en favoriser la réussite?

Voici l'expérience des deux projets menés par la médiathèque Blaise-Cendrars : le jeu de société (mis en place en 2010) et le jeu vidéo (mis en place en 2012).

\section{LE JEU DE SOCIÉTÉ À LA MÉdIATHĖQUE BLAISE-CENDRARS}

Née avant tout d'une passion forte de la part des personnels du pôle multimédia de la médiathèque, la mise en place d'un fonds de jeux de société a été rapidement soutenue par la direction de la médiathèque. À cette époque, la ville de Conflans-Sainte-Honorine ne disposait d'aucun accès aux jeux de société sur son territoire : pas de ludothèque ni d'association de jeu. Depuis, l'association «Au confluent des jeux » a été créée en 2013, à destination du public adulte.

\section{DÉBUT DU JEU}

Dès réception des premières acquisitions, nous avons mis en place des soirées jeux de société. Ce temps d'animation a lieu un vendredi par mois au sein de la médiathèque, de $18 \mathrm{~h}$ à $21 \mathrm{~h} 30$ et donc majoritairement pendant un temps de fermeture (la médiathèque ferme à $19 \mathrm{~h}$ ). C'est donc par l'animation que le fonds des jeux de société a pris de l'ampleur ; nous avons même eu l'occasion de voir des usagers apporter leurs propres jeux 
de société et animer des tables spontanément. Ces soirées sont fréquentées par trente à soixante personnes (en moyenne : quarante-cinq personnes) de tous les âges : enfants accompagnés par les parents ou grandsparents, adolescents et adultes. Il n'est pas nécessaire d'être inscrit aux services de la médiathèque pour profiter de ces soirées.

\section{SUR PLACE OU À EMPORTER ?}

En raison de contraintes d'espaces et de personnel, la médiathèque Blaise-Cendrars ne prête pas les jeux de société. En effet, nous ne disposons pas d'un espace dédié au jeu, et les jeux de société sont rangés dans une armoire vitrée se trouvant dans la salle d'étude multimédia (il y aurait conflit d'usages si les jeux étaient en accès libre). De plus, nous ne disposons pas d'assez de ressources humaines pour vérifier les boîtes de jeux à chaque retour de prêt. Ainsi, les jeux sont à utiliser durant des horaires dédiés, ce qui permet un accompagnement de la part des bibliothécaires présents, tant pour aider dans le choix du jeu que dans la mise en jeu* (installation du plateau de jeu, explication des règles et suivi de la partie si besoin).

\section{JOUER EN MÉDIATHĖQUE : ACCOMPAGNER LES COLLĖGUES}

L'arrivée des jeux de société a cependant suscité des interrogations de la part de quelques collègues, notamment sur la place que ce bien culturel pouvait avoir en médiathèque. Ainsi, une collègue témoigne : " $A u$ début, je me suis interrogée sur la place du jeu au sein de la médiathèque. Je n'étais pas contre mais le jeu me semblait avoir sa place plutôt en ludothèque. Ces dernières sont cependant moins présentes qu'il y a quelques années, et le fait que les médiathèques prennent le relais ne m'a pas convaincue dans un premier temps. Il est difficile de faire cohabiter les animations autour du jeu qui génèrent du bruit et les autres usages, plus calmes durant les heures d'ouverture. "

C'est principalement en impliquant les collègues dans le suivi des animations que leur regard a pu évoluer. Ainsi, je n'ai pas hésité à solliciter le soutien et la participation de collègues d'autres pôles de la médiathèque lors des animations jeux. Cette nouvelle activité nécessite un accompagnement 
des équipes et une préparation en amont des animations programmées. Vous pouvez pour cela préparer les jeux que vous comptez présenter au public puis proposer aux collègues des moments de jeux. Le moment " idéal » pour nous était après la pause déjeuner et avant l'ouverture au public, lors d'un moment de détente. Moins d'une heure suffit : l'idée n'est pas de jouer mais de présenter les règles du jeu et son installation. Faites deux ou trois tours de jeu maximum, et selon l'engouement (et le temps dont chacun dispose !), vous pouvez proposer de terminer la partie. En ne se sentant pas " obligés » de terminer la partie, certains seront plus à l'aise et moins angoissés. Vous évitez aussi les potentiels conflits avec les " bons » et les " mauvais » perdants! Ainsi, suite à cette mise en situation, une collègue me confie : «En participant aux soirées jeux, j'ai redécouvert le plaisir de jouer en société ». Le jeu de société est maintenant ancré dans les pratiques professionnelles de l'équipe.

\section{Du jeu de société au jeu en général...}

L'introduction des jeux de société à la médiathèque a également permis de développer d'autres pratiques au sein de la médiathèque, comme par exemple celle du jeu de rôle. Ainsi, un jeu de rôle pour adolescents est proposé lors des soirées jeux de société depuis septembre 2012. Du jeu de rôle pour adultes est également proposé de manière plus ponctuelle. Ce nouveau volet « jeux » a conduit à une réflexion étendue à la place du jeu vidéo au sein de la médiathèque.

\section{LE JEU VIDÉO À LA MÉdIATHĖQUE BLAISE-CENDRARS}

\section{PRESS START : LES DÉBUTS DU JEU VIDÉO}

Les mutations que vivaient les bibliothèques nous ont amenés à nous poser la question de la place du jeu vidéo au sein de notre structure dès 2007. Dans un premier temps, la médiathèque ne proposait que quelques jeux sur PC (dont des jeux éducatifs) à emprunter de manière indirecte (les jeux étaient sous vitrine). En parallèle, des tournois de jeux sur PC étaient organisés au sein de la salle multimédia (cinq ordinateurs disponibles). 
Ces prémices ont permis à l'équipe d'intégrer progressivement le jeu dans les pratiques de la médiathèque. Rapidement, nous avons souhaité évoluer et proposer des jeux vidéo sur consoles ancienne et nouvelle génération, tant pour la consultation sur place que pour le prêt de jeux. Cependant, ce projet de plus grande ampleur nécessitait de trouver les collègues qui seraient un véritable soutien dans l'accomplissement du projet.

\section{comment se former?}

Afin de préparer au mieux l'arrivée des jeux vidéo dans l'établissement, le personnel du pôle multimédia a participé à une formation de deux jours sur le jeu vidéo․․ L'ensemble du personnel de la médiathèque a été également vivement encouragé à suivre une journée d'étude autour du jeu vidéo afin de mieux appréhender ce bien culturel. Les collègues intéressés ont principalement été ceux qui jouent à titre personnel, mais quelques néophytes ont également souhaité participer au projet, notamment sur l'établissement d'une politique d'acquisition. De plus, un temps de formation en interne a également été mis en place sur l'utilisation des consoles : branchements, lancement du jeu, fonctionnement des manettes, etc. Un atelier pratique a suivi la démonstration. Selon les équipes et les personnes, ces formations peuvent être menées en groupe, l'idéal étant un groupe de 4 personnes afin de pouvoir jouer tous ensemble ou bien de façon individuelle. Nous étions prêts à faire jouer le public !

\section{où dans la médiathèque ?}

Notre médiathèque est le fruit du réaménagement, effectué en 1989, d'une ancienne fonderie. Deux possibilités semblaient judicieuses pour l'installation de l'espace jeux vidéo (écran de télévision et consoles) : une partie de la salle de documentation accolée à la salle multimédia ou une pièce fermée à l'étage, loin des collections. La première proposition fut retenue par l'équipe car elle permettait un meilleur suivi de l'utilisation de l'espace et offrait une meilleure cohérence car l'espace était proche des collections, à proximité des jeux de société, à consulter sur place dans la

1. Exemple de formation : < http://cnfpt.fr/trouver-formation/detail-stage?id=30095\&provenance= itineraire\&id_itineraire=21_00026 >. 
même salle. Par ailleurs, les réunions avaient également permis de mettre en évidence une remarque pertinente de la part de l'équipe jeunesse dont l'espace accueillait la salle fermée : le personnel ne se sentait pas " apte » à accueillir et orienter les usagers des jeux de façon permanente, malgré l'aide de collègues dédiés.

\section{Des consoles et des bibliothécaires}

Ainsi, le jeu, sous toutes ses formes, pourra être utilisé pour des animations. Elles sont un excellent moyen d'impliquer les collègues. En effet, au contact du public, ils se trouvent en situation de médiation et donc en " terrain connu »: le jeu devient alors plus accessible, sa place au sein de la médiathèque prend tout son sens. Cependant, nous avons veillé à ce que les collègues ne se retrouvent pas seuls (du moins dans un premier temps) mais accompagné par une personne de l'équipe à l'aise avec les jeux et connaissant les règles et les outils. Dans cette optique, nous avons proposé une première participation de type "immersion " : rester avec le binôme de collègues pour observer, être disponible et répondre aux questions puis une future participation progressivement plus active selon le ressenti du collègue. L'idée étant de faire prendre conscience à chacun de la place du jeu et de son impact au sein de la médiathèque.

Pour cela, nous avons privilégié la participation lors de notre rendez-vous mensuel "Après-midi jeux vidéo » : un vendredi après-midi par mois, toutes les consoles sont mises en même temps à disposition des usagers, sans conditions d'inscription. En effet, en raison du peu d'espace disponible et de l'absence d'espace dédié au jeu, nous ne pouvons pas envisager un accès simultané à l'ensemble des consoles en dehors de ces temps (un seul écran disponible) où des moyens matériels supplémentaires sont nécessaires (écran cathodique pour le rétrogaming* et vidéoprojecteurs viennent renforcer l'équipement). Les collègues sont donc amenés à effectuer les branchements, assurer l'aspect technique de la mise en route des consoles et des jeux, et prendre en charge la partie médiation et accompagnement des usagers. 


\section{Sur place et à emporter}

Le fonds des jeux vidéo a avant tout été pensé pour le prêt. Après un test infructueux de prêt direct des jeux vidéo (vol et détérioration importants), le choix a été fait de passer au prêt indirect. Les boîtiers des jeux ainsi que leurs livrets sont laissés en libre accès. Les usagers viennent ensuite emprunter le jeu et le personnel se charge de la transaction ; le jeu est stocké dans une pochette à part et identifié par un numéro apposé sur le jeu.

De plus, pour permettre le service de la consultation sur place, nous disposons d'un fonds spécifique, tous Pan European Game Information (PEGI, système d'évaluation européen des jeux vidéo) confondus. Cela suppose un achat en plusieurs exemplaires du même jeu, et nous permet d'éviter la frustration chez nos usagers, de ne pas pouvoir emprunter le jeu testé à la médiathèque.

\section{Publics}

Mais la venue de ce média a permis d'ouvrir la médiathèque à un nouveau public qui ne fréquentait pas les lieux jusqu'alors. Ce public se compose principalement d'adolescents et de jeunes adultes, mais aussi de familles. De plus, nous pouvons aussi faire découvrir le jeu et initier le public déjà fréquentant et non familier du support.

\section{APRÈs Le JEU}

\section{L'échange après l'expérience de jeu}

À l'issue d'un temps consacré au jeu, en interne, lors d'animations ou bien durant les temps de médiation au public, il est souhaitable de dialoguer avec les collègues qui le désirent. Ces échanges vont permettre de faire un retour sur l'expérience, de répondre aux questions éventuelles entre collègues. Ces temps d'échanges peuvent avoir lieu tout de suite après le jeu ou bien à des moments dédiés comme par exemple lors des réunions de l'ensemble du personnel. Il ne faut pas que ces temps d'échanges cessent, mais ils pourront s'espacer et devenir plus succincts lorsque le projet aura trouvé ses marques au sein de la médiathèque et des équipes. 


\section{Fin de partie ?}

Ainsi qu'il est d'usage pour tous les types d'animation, il est nécessaire d'effectuer un bilan (même rapide) des moments de jeux et de les communiquer à l'ensemble de l'équipe afin que l'évolution du projet et sa mise en place prennent une dimension plus concrète dans les esprits. Cela va aussi permettre d'être plus attentif aux retours des collègues et des usagers, et par là même, d'affiner le projet et d'adapter les chartes et autres règles aux usages du public. Il en va de même pour le service de prêt qu'il sera nécessaire d'évaluer, comme pour les autres collections, pour façonner la politique documentaire et mieux connaître et orienter son public.

\section{L'équipe et les jeux : conseils}

Choisir le jeu en fonction des goûts du collègue et du public visé (ou faire l'inverse et choisir le collègue en fonction du public) selon ses habitudes et compétences à travailler avec un public jeunesse, adulte ou spécifique ;

Présenter les jeux en amont aux collègues qui vont vous aider lors de votre animation ;

Établir des binômes de compétences pour le démarrage des actions d'animation ;

Prévoir des temps de retour et dialoguer avec les collègues après l'animation : ressenti, difficultés, éléments positifs, choses à modifier pour la prochaine fois...;

Accompagnement, formation, patience, dialogue sont de mise : la médiation culturelle doit se faire autant avec les usagers qu'avec les collègues. 


\section{ENCADRÉ 《 ...ET ILS EN PENSENT QUOI, LES COLLĖGUES ? »}

Questions aux collègues de la médiathèque Blaise-Cendrars de Conflans-Sainte-Honorine : Catherine Benod (bibliothèque adulte), Laurence Deletoille (responsable de la bibliothèque jeunesse), Olivia Le Strat (services aux publics) et Frédérique Nancey (bibliothèque jeunesse).

Élise Ybled : Êtes-vous joueur (jeux de société et jeux vidéo) ? Participez-vous aux animations autour du jeu ? Empruntez-vous des jeux?

Catherine Benod : Je suis joueuse occasionnelle pour les jeux de société mais joueuse régulière pour les jeux vidéo. J'essaie au moins d'aider les collègues sur une soirée jeux de société par an et j'aide souvent pour encadrer les jeux vidéo. Je n'emprunte pas de jeux vidéo (collection personnelle importante et durée de prêt trop courte pour moi) mais par contre j'emprunte des jeux de société, surtout pour mes enfants.

Laurence Deletoille : Non, je ne suis pas joueuse à la base mais en participant aux soirées jeux de société, j'ai redécouvert le plaisir de jouer en société. Je ne suis pas du tout joueuse de jeux vidéo et n'emprunte pas de jeux.

Olivia Le Strat : Je joue un peu aux jeux de société, et plus régulièrement aux jeux vidéo (notamment avec mes enfants, mais aussi avec des amis). J'emprunte des jeux pour mes enfants. Je participe aux soirées jeux de société et j’aide les collègues ; je cadre également les moments jeux vidéo en accès libre pendant les vacances scolaires.
Frédérique Nancey : Je participe aux soirées jeux de société organisées par la médiathèque et j'aide les collègues. Je joue beaucoup aux jeux de société, surtout avec mes enfants (mon mari est joueur et créateur de jeux de société). Par contre, je ne joue pas aux jeux vidéo mais je cadre parfois les moments de jeux en accès libre pendant les vacances scolaires.

EY : Comment avez-vous ressenti l'arrivée des jeux de société (juin 2010) et des jeux vidéo (mai 2012) au sein de la médiathèque (dans le fonds et en consultation sur place)?

Catherine Benod : L'arrivée des jeux de société a été positive pour moi parce que c'est très convivial et que le public familial a pu se retrouver dans la médiathèque. La relation avec les usagers était différente et moins institutionnelle. J'ai aussi pu me replonger dans un univers que je ne connaissais plus trop et découvrir de nouveaux jeux. On a aussi touché un nouveau public. Je n'étais pas présente pour l'arrivée des jeux vidéo (congé maternité) mais j'étais ravie de la présence de ce nouveau support qui était en phase avec ma pratique personnelle. On colle aux pratiques des usagers et on s'adapte.

Laurence Deletoille : L'arrivée des jeux a soulevé un problème d'espace dédié : il y a conflit des usages. Mais comme il n'y a pas de ludothèque dans la ville, la médiathèque a pu combler ce manque.

Olivia Le Strat : Au début, je me suis interrogée sur la place du jeu au sein de la médiathèque. Je n'étais pas contre mais 
le jeu me semblait avoir sa place plutôt en ludothèque. Ces dernières sont cependant moins présentes qu'il y a quelques années, et le fait que les médiathèques prennent le relais ne m'a pas convaincue dans un premier temps. Il est difficile de faire cohabiter les animations autour du jeu qui génèrent du bruit et les autres usages, plus calmes durant les heures d'ouverture. Les soirées jeux de société se déroulant hors du créneau d'ouverture me posent moins de problème.

Frédérique Nancey : J'ai plutôt bien vécu l'arrivée de ces nouveaux fonds, mais le problème de la place du fonds dans l'espace a été très problématique pour moi car elle a soulevé plein d'autres questions, notamment celle du bruit qu'on ne peut pas éviter pendant le jeu. La médiathèque n'était pas prévue pour accueillir le jeu (ancienne fonderie réaménagée en médiathèque en 1989). Par contre, je salue le travail des collègues sur la mise en valeur du fonds et sur la régularité des animations qui font écho à celles organisée par la bibliothèque jeunesse.

EY : Après maintenant quelques années de fonctionnement, votre opinion a-t-elle évolué ? Comment voyez-vous le jeu dans la médiathèque aujourd'hui ?

Catherine Benod : Je trouve toujours que le jeu sous toutes ses formes a sa place dans la médiathèque et qu'il nous permet de toucher de nouveaux publics, surtout les enfants qui ne viendraient pas pour autre chose que les jeux. Il y a eu un vrai travail mené par les collègues de l'espace multimédia pour faire intégrer les règles aux enfants sur l'utilisation de l'espace (environ une année de travail au quoti- dien) et tout se déroule beaucoup mieux maintenant. En revanche, sur les grosses animations (tournois, après-midi jeux, soirées jeux de société), il y a un conflit d'usages (jeux et travail) de l'espace au sein de la médiathèque : l'absence d'un espace dédié aux jeux pose alors un vrai problème.

Laurence Deletoille : Les jeux se sont intégrés dans les pratiques de la médiathèque. En revanche, certaines acquisitions me posent problème (exemple : Grand Theft Auto (ou GTA), PEGI 18 est sujet à controverse). Le fait qu'il y ait du jeu en dehors des heures d'ouverture de la médiathèque permet aux usagers de créer un lien social autre, entre eux et avec les bibliothécaires. On est dans le « jouer ensemble » en dehors de la cellule familiale et amicale. Certains usagers viennent seuls et trouvent une table de jeux : une mixité se crée alors entre les joueurs. Les gens reviennent aux soirées : les soirées jeux de société deviennent un rendez-vous régulier. Ils restent souvent plus longtemps aux moments jeux, plus qu'ils ne seraient restés à la médiathèque pour consulter d'autres documents.

Olivia Le Strat : J'intègre plus les jeux, et notamment les animations, dans les pratiques de la médiathèque. On touche tous les publics, ils peuvent s'emparer du média et le découvrir, comme ils le feraient avec un livre, un CD, un DVD. La médiathèque se doit d'offrir l'accès à la culture, et le jeu est un bien culturel à part entière : c'est là que ma vision a évolué. Je trouve cependant qu'il faudrait développer l'aspect de réflexion autour du jeu, sous forme de débats ou de ren- 
contres pédagogiques : le jeu est un réel fait de société et soulève beaucoup d'interrogations auxquelles nous devons proposer plus de temps de parole.

Frédérique Nancey : Au niveau de l'évolution, ça me paraît compliqué d'emprunter les jeux de société car les manipulations de vérification sont très lourdes. Les animations sont très bien et en nombre suffisant. Il faudrait peut-être plus dé- velopper des moments de débat et de médiation avec les parents notamment (autour du jeu de société et du jeu vidéo). Pour les jeux vidéo, j'aimerais avoir plus de temps pour découvrir les jeux entre collègues. Quand les usagers recherchent un jeu, ils connaissent souvent la référence et requièrent peu notre expertise ; mais le catalogue détaillé et le PEGI me permettent d'affiner les recherches et d'ouvrir les usagers à d'autres jeux. 
3

\section{LE CADRE JURIDIQUE DU JEU EN BIBLIOTHĖQUE : ACQUISITIONS, CONSULTATION, PRÊT \\ Thomas \\ Fourmeux et \\ Lionel Maurel}

Activité sociale par excellence, le jeu a naturellement vocation à faire l'objet d'usages collectifs. Contrairement aux usages personnels, s'inscrivant dans le cadre du " cercle de famille », la plupart des usages collectifs des jeux qui peuvent avoir lieu dans une bibliothèque publique sont susceptibles d'entrer en contradiction avec les règles du droit d'auteur, qui les soumettent normalement à une autorisation préalable des titulaires de droits $^{1}$. Dans le domaine du livre, la loi est intervenue pour instaurer une forme d'équilibre permettant à des activités comme le prêt public de se déployer, en contrepartie d'une rémunération des auteurs et des éditeurs. Mais ce type de solution n'est malheureusement pas applicable en l'état du droit existant aux différents types de jeux.

La mise à disposition en bibliothèque de jeux physiques, par exemple des jeux de société, peut déjà poser un certain nombre de problèmes juridiques, mais la situation est exacerbée avec les jeux vidéo, dont les usages se développent sous de nombreuses formes en bibliothèque, sans disposer d'une base légale véritablement solide ${ }^{2}$. Cette situation n'est à vrai dire pas nouvelle en bibliothèque (elle concerne notamment depuis longtemps le prêt de CD musicaux) et il ne s'agit pas d'affirmer que le jeu n'aurait pas sa place dans les bibliothèques pour des raisons juridiques.

Mais les professionnels doivent être conscients de ce décalage entre le droit et la pratique lorsqu'ils mettent en place de nouveaux services autour du jeu en bibliothèque.

1. Calimaq, « Usage collectif et usage privé en bibliothèque : essai de clarification », S.I.Lex, 12 décembre 2010. [En ligne] : < http://scinfolex.com/2010/12/12/usage-collectif-et-usage-priveen-bibliotheque-essai-de-clarification/ >.

2. Françoise Legendre, Jeu et bibliothèque : pour une conjugaison fertile, Rapport $\mathrm{n}^{\circ}$ 2015-009 de l'Inspection générale des bibliothèques, février 2015, pp. 89-90. [En ligne] : < http://www. enssib.fr/bibliotheque-numerique/documents/65198-jeu-et-bibliotheque-pour-une-conjugaison-fertile.pdf >. 


\section{COMPLEXITÉ DE LA NATURE JURIDIQUE DES JEUX}

Déjà sous forme physique, les jeux ont une nature juridique relativement complexe. Concernant les jeux de société, les règles peuvent être protégées par le droit d'auteur si elles sont suffisamment originales, mais beaucoup de mécanismes de jeu sont trop abstraits pour ouvrir droit à une protection. L'esthétique d'un jeu (plateau, figurines, etc.) peut en revanche plus facilement être protégée par le biais des dessins et modèles et son nom déposé comme marque ${ }^{3}$. Plusieurs éléments de protection se juxtaposent souvent en matière de jeu.

La nature juridique des jeux vidéo est encore plus complexe et elle est restée longtemps incertaine. Les jeux vidéo sont en effet des œuvres composites combinant plusieurs éléments de nature différente : logiciel, scénario, éléments graphiques, musiques, etc. Leur réalisation est généralement le fait de plusieurs auteurs, travaillant pour des éditeurs spécialisés. Face à cette situation, plusieurs notions juridiques peuvent correspondre au cas du jeu vidéo : soit œuvre collective, soit œuvre de collaboration. La première qualification emporte que les éditeurs disposeraient de l'essentiel des droits sur les jeux vidéo, tandis que la seconde au contraire en laisserait la titularité initiale aux différents auteurs.

Après un certain nombre de flottements, la jurisprudence semble à présent pencher pour le statut d'œuvre de collaboration ${ }^{4}$. L'alternative n'a pas à vrai dire une incidence directe sur les bibliothèques, puisque dans les deux cas, on est en présence d'œuvres protégées. Mais le choix de l'œuvre de collaboration rend sans doute plus complexe l'émergence d'offres adaptées aux bibliothèques, car elle implique l'accord d'un plus grand nombre d'acteurs (éditeurs et multiples auteurs).

3. Institut national de la propriété intellectuelle (INPI), Comment protéger mon jeu de société. [En ligne] : < http://www.inpi.fr/fr/l-inpi/la-propriete-industrielle/comment-proteger-vos-creations. html\#c330 >.

4. Géraldine Laly, « Le jeu vidéo suscite toujours autant d'émois juridiques », Legavox, 20 janvier 2012. [En ligne] : < http://www.legavox.fr/blog/maitre-geraldine-laly/video-suscite-toujours-autant-emois-7510.htm\#.VD0R9md_vdV >. 


\section{ACQUISITION DES JEUX : LE DISTINGUO ENTRE MODĖLE "в TO B" ET "B TO C"5}

Quand une bibliothèque met des livres physiques en prêt, elle acquiert via un fournisseur les mêmes ouvrages que ceux qui sont vendus aux particuliers, sans que l'éditeur n'ait à proposer une offre spéciale aux bibliothèques. Cette possibilité découle de la loi du 18 juin 2003 qui a instauré une licence légale* pour le prêt public des livres en France, en contrepartie d'une rémunération versée aux auteurs et aux éditeurs ${ }^{6}$. Mais ce mécanisme ne vaut que pour le livre papier et il n'a pas été étendu à d'autres types de supports d'œuvres (CD musicaux, DVD, etc.), ni aux ressources numériques.

Or une directive européenne adoptée en $1992^{7}$ a de son côté clairement établi que le prêt public ou la location d'une œuvre protégée, de quelque nature qu'elle soit, relèvent du droit exclusif d'autoriser ou d'interdire des titulaires de droits. Il en résulte que les bibliothèques ne peuvent pas acheter les œuvres destinées aux simples consommateurs individuels (modèle "B to C"). Elles doivent passer par des offres spécialement dédiées aux établissements et aux usages collectifs (modèle “B to B"). On sait qu'en matière de CD musicaux, aucune offre de ce type n’a émergé et que les bibliothèques prêtent ce type de supports sans autorisation explicite de la part des titulaires de droits. Pour les DVD en revanche, des intermédiaires (les centrales d'achat comme Ateliers diffusion audiovisuelle [ADAV], Colaco, CVS, etc.) se sont positionnés pour négocier le droit de prêt et de consultation auprès des titulaires de droits et faire des offres aux bibliothèques.

\section{LES JEUX PHYSIQUES}

En ce qui concerne les jeux physiques, les œuvres achetées par les ludothèques sont les mêmes que ceux que le grand public acquiert. Théoriquement, des usages comme le prêt public de tels objets sont bien couverts par les droits exclusifs et ils devraient faire l'objet d'une autorisation des

5. "B to C" pour Business to Consumer et "B to B" pour Business to Business.

6. Ministère de la Culture, Droit de prêt. [En ligne] :< http://www.droitdepret.culture.gouv.fr/ >.

7. Commission européenne, Droit de prêt et de location. [En ligne] : < http://ec.europa.eu/internal_market/copyright/rental-right/index_fr.htm >. 
titulaires, même si dans la pratique, beaucoup d'établissements privés comme publics, proposent ce type de services.

\section{LES JEUX VIDÉO}

Pour le jeu vidéo, la justice française dès 2004 s'est explicitement prononcée par une décision Nintendo ${ }^{8}$ sur le fait que la location était un acte couvert par la directive de 1992, et le raisonnement serait le même en ce qui concerne le prêt public, même effectué à titre gratuit.

À l'instar des DVD, certains fournisseurs comme Circle, CVS ou Colaco se sont positionnés sur les jeux vidéo (par extension de leurs activités sur les anciens CD-ROM), en négociant les droits auprès des éditeurs. Cela explique notamment la différence de prix entre le prix public du jeu et le prix fixé pour les collectivités, le droit de prêt étant répercuté sur le coût d'acquisition du support. Toutefois contrairement aux DVD, il semble qu'il n'y ait pas de garantie que les droits aient été clairement négociés à des fins de consultation ou de prêt en bibliothèque. Par ailleurs, l'offre disponible est bien moins riche que l'offre disponible pour le grand public.

En l'absence de licence légale, il peut arriver néanmoins que les bibliothèques bénéficient d'offres spécialement conçues pour les usages collectifs. Un intermédiaire comme la plate-forme Steam par exemple propose aux cybercafés des licences ${ }^{9}$ pour la mise à disposition de leurs clients des jeux vidéo en ligne, avec un prix variant selon le nombre de postes. Certaines bibliothèques peuvent souscrire à ce type d'offres pour proposer des services similaires ${ }^{10}$, ce qui tend à montrer que des offres adaptées pourraient voir le jour sur des bases contractuelles.

8. Cour de Cassation, Décision Nintendo, 27 avril 2004. [En ligne] $:<\mathrm{http} / / /$ www.legifrance. gouv.fr/affichJuriJudi.do?oldAction=rechJuriJudi\&idTexte=JURITEXT000007047511\&fastRe qId $=1170119201>$.

9. Voir Valve Cyber Espace Programme : < http://cybercafe.e-dg.eu/index.php?aff=prix >

10. Voir par exemple à la Médiathèque de Pierrevives : < http://pierresvives.herault.fr/page-standard/steam >. 


\section{CONSULTATION SUR PLACE ET PRÊT DE JEUX EN BIBLIOTHĖQUE}

La consultation d'un jeu en bibliothèque peut être assimilée de prime abord à une forme de représentation d'une œuvre au sens de l'article 122$2^{11} \mathrm{du}$ Code de la propriété intellectuelle (CPI). Or, le droit de représentation est normalement un droit exclusif de l'auteur concernant l'exploitation de son œuvre. Mais il importe de faire une distinction entre les jeux physiques, type jeux de société, et les jeux vidéo, dont la consultation passe par des écrans.

\section{LA CONSULTATION SUR PLACE}

En effet, la directive de 1992 sur le droit de prêt public et la location précise explicitement qu'elle n'est pas applicable aux actes simples de mise à disposition à des fins de consultation sur place des œuvres réalisés dans les emprises des bibliothèques. On en déduit par exemple que le fait de lire un ouvrage papier dans une bibliothèque reste un acte libre, non soumis au droit d'auteur. La situation est la même pour l'acte de jouer à un jeu de société dans les emprises d'une bibliothèque. Ce type d'usage est autorisé et ce n'est que le prêt public du même objet qui peut soulever des difficultés vis-à-vis des règles du droit d'auteur.

Il en est ainsi, car le simple fait de jouer à un jeu de société ne constitue pas une " communication au public ", constitutif d'une représentation. Mais les choses changent dès lors qu'intervient la médiation d'un écran, impliquant une diffusion qui constitue une représentation, même si le destinataire est un individu isolé. S'exerçant dans les murs de la bibliothèque, elle est à distinguer de la représentation privée dans le cadre du " cercle de famille », qui ne nécessite pas l'accord de l'auteur, en vertu d'une des exceptions au droit d'auteur fixée par l'article 122-5 du CPI ${ }^{12}$. Toutefois, notons que la consultation sur place en bibliothèque semble

11. Légifrance, Code de la propriété intellectuelle. [En ligne] : < http://www.legifrance.gouv.fr/affichCodeArticle.do;jsessionid=E81534BCD7E55754AE5756A09902031F.tpdjo06v_1?idArticle=LEGIART I000006278904\&cidTexte=LEGITEXT000006069414\&dateTexte=20141013 > .

12. Ministère de la Culture et de la Communication, Les exceptions au droit d'auteur. [En ligne] : < http://www.culture.gouv.fr/culture/infos-pratiques/droits/exceptions.htm >. 
actuellement tolérée aussi bien par les éditeurs de jeux vidéo que par les constructeurs de consoles. De même, les pratiques de prêt en bibliothèque, qu'elles portent sur des jeux vidéo (CD, cartouche, etc.) ou sur des appareils type consoles ne peuvent théoriquement se faire sans l'accord des titulaires de droits.

En réalité, les conditions générales d'utilisation (CGU)* portent plus spécifiquement sur le logiciel qui fait fonctionner la console que sur le matériel lui-même ou bien sur le contenu quand il s'agit d'un jeu vidéo. Par exemple, l'éditeur de jeux Ubisoft stipule explicitement le caractère particulier de l'usage du jeu : "Les services sont destinés à une utilisation à des fins personnelles uniquement et vous ne devez en aucun cas en faire un quelconque usage commercial. Vous vous engagez à ne pas vendre, louer ou commercialiser le contenu $»^{13}$.

La situation actuelle s'explique en grande partie par le fait que la question du prêt et de la mise à disposition des jeux en bibliothèque ne constituent pas une préoccupation fondamentale pour les éditeurs de jeux vidéo qui ne les identifient généralement pas comme un marché.

De même, les CGU de certains fabricants de consoles contribuent à entretenir l'idée d'un flou juridique ${ }^{14}$. À l'image d'Ubisoft, Sony ignore l'existence du jeu vidéo dans les établissements de lecture publique. En effet, les CGU de ses consoles ne mentionnent pas le prêt ${ }^{15}$ : " Vous n'êtes pas autorisé à louer à bail, donner en location, concéder sous licence, publier, modifier, corriger, adapter ou traduire le logiciel système ».

Si les CGU n'interdisent pas explicitement le prêt, elles ne l'autorisent pas non plus. Dans l'idéal, il conviendrait de demander directement l'autorisation de Sony pour utiliser sa console dans un lieu dédié à l'usage collectif. Certains établissements de lecture publique ont déjà sollicité Nintendo pour avoir l'autorisation d'utiliser ses consoles dans le cadre d'une animation. Mais les bibliothécaires n'ont aucune garantie d'obtenir une réponse et dans une telle situation, on ne peut appliquer l'adage " qui ne dit mot consent ».

13. Ubisoft, Conditions d'utilisation des services d'Ubisoft. [En ligne] : < http://www.ubi.com/fr/Info/ Info.aspx?tagname $=$ TermsOfUse $>$.

14. Enssib, Questions ? Réponses !, 9 septembre 2008. [En ligne] : < http://www.enssib.fr/content/bonjour-quel-regime-est-soumis-le-pret-de-jeux-video-pour-une-bibliotheque-en-france-est-ce >.

15. Sony, Contrat de licence de logiciel système applicable au système Playstation $® 4$. [En ligne] : $<$ http://www.scei.co.jp/ps4-eula/ps4_eula_fr.html >. 
On retrouve la même ambiguïté en ce qui concerne les consoles portables. Nintendo rappelle par exemple dans les CGU le caractère individuel de l'usage de la console : « Le logiciel est sous licence, non vendu et réservé à un usage personnel et non commercial de votre Nintendo 3DS » ${ }^{16}$. Or si le caractère non commercial des bibliothèques est relativement évident, cela est moins vrai pour l'usage personnel, comme nous l'avons vu plus haut.

Le cadre juridique du jeu vidéo en bibliothèque n'est donc pas à l'avantage des bibliothèques.

\section{USAGES NUMÉRIQUES, TABLETTES ET APPLICATIONS}

De plus en plus de bibliothèques font l'acquisition de tablettes. Les professionnels organisent et animent des ateliers avec ces appareils pour faire découvrir des applications. Une des formes les plus plébiscitées est par exemple l'heure du conte numérique ${ }^{17}$. L'accord des titulaires de droits est pourtant théoriquement nécessaire pour ce type d'animation. La projection d'une application au moyen d'un vidéoprojecteur dans l'enceinte de la bibliothèque s'apparente en effet à une forme de représentation publique de l'œuvre.

Tout comme pour les jeux vidéo et les consoles, les applications sont à distinguer des appareils de lecture. Les tablettes sont conditionnées par des CGU qui ne sont pas favorables à l'usage en bibliothèque. Le fabricant Samsung précise que "vous ne pouvez ni louer, donner à bail, prêter ou concéder en sous-licence le logiciel ${ }^{18}$. Si l'on s'en tient strictement au point de vue des CGU, les bibliothèques ne devraient donc pas prêter les tablettes à leurs usagers ${ }^{19}$. Néanmoins, les fabricants tolèrent largement ces usages collectifs, quand ils ne les encouragent pas en faisant des

16. Nintendo, Conditions générales d'utilisation. [En ligne]: < https://www.nintendo.com/consumer/ info/en_na/docs.jsp >.

17. La Petite Bibliothèque Ronde, Heure du conte numérique, avril 2012. [En ligne] : < http://blog. petitebiblioronde.com/la-vie-de-la-bibliotheque/heure-du-conte-numerique-1177 >.

18. Contrat de licence utilisateur final tablette Galaxy Tab S Samsung.

19. Université de technologie de Belfort-Montbéliard, Prêt de tablettes. [En ligne] : < http://www. utbm.fr/espace-bibliotheque/services/tablettes.html >. 
offres spéciales pour le secteur scolaire ${ }^{20}$ (mais pas encore explicitement pour les bibliothèques).

Concernant les applications utilisées avec les tablettes, l'usage en bibliothèque soulève également des problèmes de propriété intellectuelle. Les bibliothèques disposant d'un parc de tablettes installent souvent une application achetée une seule fois sur plusieurs appareils, en utilisant les marges de manœuvre ouverte par les magasins d'application. Or il y a ici une véritable confusion entre le modèle "B-to-B" et "B-to-C", détaillé plus haut. Outre le problème de rémunération de la création que pose cette pratique, elle est de plus en plus perçue comme une atteinte au droit d'auteur des éditeurs et des auteurs d'applications ${ }^{21}$. Le modèle de l'application utilisée dans un contexte d'usage collectif reste à développer $^{22}$. En l'absence de tout cadre légal couvrant les usages collectifs, une des alternatives consiste à recourir à une solution contractuelle avec les éditeurs pure player*.

\section{UN CONTEXTE JURIDIQUE FRAGILE POUR LES BIBLIOTHĖQUES}

Force est de constater que les bibliothèques développent souvent des services autour du jeu en dehors de tout cadre légal. Il s'agit en réalité d'une tolérance de fait accordée par les éditeurs qui pourraient décider de remettre en cause cet équilibre précaire. Par ailleurs, cette situation ne favorise pas la mise en place d'offre adaptée aux bibliothèques. Le rapport Lescure avait d'ailleurs pointé le retard en la matière ${ }^{23}$. Pour faire évoluer la situation, il serait nécessaire d'ouvrir un dialogue avec les acteurs de l'industrie du jeu pour aboutir à une situation qui garantisse la possibilité pour les bibliothèques de proposer ce type de services.

20. Voir par exemple Apple, iPad in Education : < https://www.apple.com/education/ipad/ >.

21. La Souris qui raconte, Ah le vide juridique, février 2014, [En ligne] $:<$ http://www.lasourisquiraconte.com/blog/ah-le-vide-juridique\#comments >.

22. Il commence par exemple à exister en ce qui concerne la presse, avec l'application pour iPad développée par lekiosque.fr : [En ligne] : < http://fr.slideshare.net/aioutz/experience-risomipad-lekiosquefr-arald-17-juin-2011 >.

23. Pierre Lescure, Rapport de la Mission Acte II l'exception culturelle à l'ère du numérique, 2013, [En ligne] : < http://www.culturecommunication.gouv.fr/Actualites/En-continu/Culture-acte2-80-propositions-sur-les-contenus-culturels-numeriques >. 
Il ne s'agit pas de mettre un terme à toutes ces activités, mais d'être conscient du contexte juridique fragile dans lequel les bibliothèques proposent ce service à leurs usagers. La situation actuelle semble confortable pour les établissements, mais le vide juridique n'existant pas, celle-ci ne protège pas la dimension collective de cet usage à la différence de la loi sur le droit de prêt du livre.

Le développement progressif du jeu en bibliothèque pourra peut-être conduire à de nouvelles perspectives. La directive européenne de $1992^{24}$ couvre l'ensemble des œuvres. Il suffirait de la transposer en intégrant le jeu vidéo en s'appuyant sur la loi sur le droit de prêt du livre. Toutefois, cette transposition nécessiterait de mettre en place un mécanisme de rémunération des auteurs. Qui supporterait les coûts de cette extension de la loi sur le droit de prêt ? Par ailleurs, ce type de mécanisme se fonde sur des œuvres sur support physique. Il n'est pas certain qu'il soit reconductible dans l'environnement dématérialisé. Or, l'industrie du jeu vidéo abandonne de plus en plus les supports physiques. 


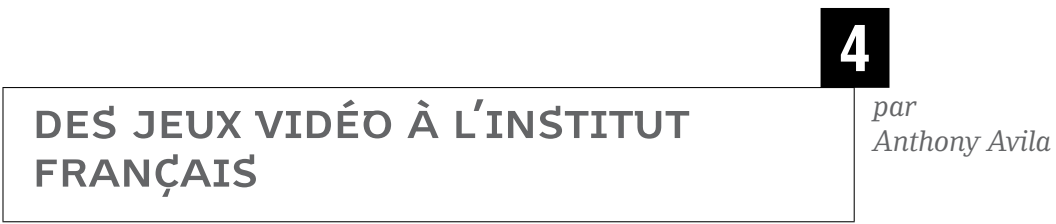

L'Institut français est l'un des principaux outils de la diplomatie culturelle française, et doit contribuer au rayonnement culturel de la France à l'étranger ${ }^{1}$. Dans la pratique, l'une de ses activités principales est l'apprentissage de la langue française, ainsi que la promotion de la culture française.

Le jeu vidéo, bien que né et développé majoritairement entre les ÉtatsUnis et le Japon, a en France une place toute particulière. Ubisoft, éditeur français de jeux vidéo au chiffre d'affaires annuel de $1256 \mathrm{M} €$ en $2013^{2}$ est le seul à ne pas venir d'un de ces deux autres pays dans le Top 5 mondial des éditeurs de jeux vidéo ${ }^{3}$. Les seuls autres Européens à entrer dans le Top 10 sont les Allemands de Koch Media qui ne produisent pas la moitié de ce chiffre d'affaires. Dans le Top 20, on retrouve trois autres éditeurs français : Just for Games, Focus Home Interactive et Big Ben. Le territoire français est par ailleurs très bien équipé en écoles de jeux vidéo, et de nombreux développeurs français travaillent également partout dans le monde, à l'image de Christophe Balestra, devenu co-président de Naughty Dog dont le dernier jeu, The Last of Us, s'est vendu à plus de 6 millions d'exemplaires ${ }^{4}$. D'autres studios, comme Arkane (plus de 3 millions d'exemplaires vendus pour Dishonored $)^{5}$, sont édités par des Américains et développent en France. Certaines créations françaises sont également considérées comme des inspirations par des développeurs du monde entier : les Japonais Fumito Ueda (ICO, Shadow of the Colossus) et Hideo Kojima (la série Metal Gear) citent souvent le jeu Another World du Français Éric Chahi comme une influence majeure dans leur travail.

1. [En ligne] : <http://www.institutfrancais.com/fr/faites-notre-connaissance- $0>$.

2. [En ligne] : <http://gsl100.com/classement/top-10-des-editeurs-de-jeux-videos $>$.

3. Syndicat des éditeurs de logiciels de loisirs, L’Essentiel du jeu vidéo, février 2014, p. 20. [En ligne] : < http://www.essentiel-jeu-video.fr/media/pdf/EJV_01_FR.pdf > .

4. [En ligne] : $<$ http://www.vgchartz.com/gamedb/?name=last+of +us $>$.

5. [En ligne] : <http://www.vgchartz.com/gamedb/?name=dishonored $>$. 
Ainsi, le jeu vidéo peut entrer dans la mission de promotion de la culture française de l'Institut français, tant il est un élément important de la création contemporaine de notre pays.

\section{JEUX VIDÉO ET CULTURE FRANÇAISE DANS LES INSTITUTS FRANÇAIS}

Certains instituts ne s'y sont pas trompés et ont déjà entamé un travail dans ce sens. L'Institut français et le Goethe Institut de Tallin en Estonie ont conjointement mis en place une exposition sur le jeu vidéo en $2013^{6}$ tandis que la médiathèque de l'Institut français a installé un petit espace de consultation sur place de jeux vidéo français ${ }^{7}$. L'Institut français de Naples $^{8}$ en Italie, celui de Valence en Espagne ou celui de d'Helsinki en Finlande ${ }^{9}$ ont également mis en place ce type d'espace ludique. L'Institut français de Tokyo au Japon a, quant à lui, mis en place en 2013 une rencontre entre créateurs de jeux vidéo français et japonais ${ }^{10}$ et l'Institut français de Jérusalem propose des activités autour du jeu vidéo dans le cadre de rendez-vous hebdomadaires pour les enfants ${ }^{11}$.

\section{LA PRATIQUE DU FRANÇAIS PAR LE JEU VIDÉO : QUELQUES PISTES}

Cependant, l'apprentissage du Français langue étrangère (FLE) reste l'activité principale auprès du public des instituts français, et il serait intéressant d'utiliser le jeu vidéo dans ce cadre.

6. [En ligne] : < http://www.ambafrance-ee.org/Exposition-consacree-aux-jeux >.

7. [En ligne] :< http://www.ife.ee/fr/a/tallinn-antenne/mediatheque/espace-jeuvideo >.

8. [En ligne] : < http://institutfrancais-napoli.com/fr/ifn/mediatheque/espace-ludotheque $>$.

9. [En ligne] : < http://www.france.fi/category/helsinki/mediatheque-et-livre >.

10. [En ligne] : < http://www.institutfrancais.jp/tokyo/fr/events-manager/eric-chahi-rencontre >.

11. [En ligne] : < http://www.ccfgary-jerusalem.org.il/?s=jeux+video >. 


\section{UN FONDS DE JEUX VIDÉO}

S’il existe peu de jeux sérieux* ou ludo-éducatifs adaptés au FLE ${ }^{12}$, on peut cependant envisager une approche moins directe : de la même façon que les médiathèques des instituts français proposent des livres ou des films en français pour que les apprenants puissent pratiquer la langue, le même genre d'offre peut être mise en place pour les jeux vidéo. En effet, nombreux sont les jeux où le texte et sa compréhension ont une place centrale, comme les jeux d'aventure* par exemple. De plus, les jeux sont souvent de grands motivateurs pour l'apprentissage et permettent de pratiquer dans un cadre non scolaire.

La plupart des jeux français sur les consoles modernes sont compatibles avec les consoles des autres pays (Playstation 3, Playstation 4), mais pas toutes. Ainsi, les jeux français sur console Nintendo 3DS ne fonctionneront qu'en Europe, par exemple. Le problème est que souvent, la langue ne dépendra pas du jeu mais de la configuration de la machine dans laquelle il est utilisé. Il semble alors complexe de proposer des jeux de ce type en prêt sans une médiation lourde en parallèle.

\section{DES CONTENUS DÉMATÉRIALISÉS}

Une autre piste pourrait être explorée autour de contenus dématérialisés. L'association M05.COM ${ }^{13}$ avait entamé en 2013 des discussions avec la société DotEmu ${ }^{14}$ pour construire une offre de jeux spécifique aux médiathèques. Cette entreprise propose un large catalogue de jeux plus ou moins anciens en langue française. Il serait possible de leur proposer de composer une offre spécifique aux instituts français, pour leurs élèves et leurs publics, pour qu'ils puissent bénéficier de jeux français ou francophones pour pratiquer la langue.

12. Thomas Constant, Quelles utilisations des jeux sérieux pour l'apprentissage de la langue française ?, Académie de Créteil, 8 décembre 2011. [En ligne] : < http://jeuxserieux.ac-creteil. $\mathrm{fr} / \mathrm{p}=1141>$.

13. Association pour la protection et la mise en valeur du patrimoine vidéoludique. [En ligne] : $<$ http://mo5.com $>$.

14. [En ligne] : < http://www.dotemu.com/fr >. 


\section{LES JEUX COMMUNAUTAIRES}

Une autre piste se trouve dans les jeux multi-joueurs ou les jeux en ligne. Un des meilleurs moyens de pratiquer la langue est de le faire dans le cadre d'une activité de groupe avec d'autres personnes. Jouer à des jeux à plusieurs en français ou avec des joueurs français peut être une bonne piste. Dofus ${ }^{15}$ et Wakfu ${ }^{16}$ sont des créations françaises d'Ankama, des Massively Multiplayer Online Role Playing Games (MMORPG)*, ou jeux de rôle massivement multi-joueur en français, ce sont en fait des jeux sur Internet permettant de jouer avec de très nombreux joueurs en simultané. Ils sont "persistants ", c'est-à-dire que le jeu ne s'arrête pas quand le joueur se déconnecte, il y a toujours des événements qui se déroulent dans l'univers, mettant en scène d'autres joueurs. Y jouer sur un serveur français peut être un très bon moyen de pratiquer la langue. Des jeux comme Hordes $^{17}$ ou Mush ${ }^{18}$ de Motion Twin ${ }^{19}$, un autre développeur français, sont d'autres options intéressantes. Ce sont des jeux communautaires qui se jouent uniquement dans son navigateur internet et dans lesquels il faut absolument communiquer et jouer en coopération avec les autres participants pour espérer gagner la partie.

Une possibilité encore plus intéressante serait de faire jouer ensemble des apprenants de pays différents à ce genre de jeux. Leur langue commune serait alors le français et la richesse des échanges n'en serait que plus importante encore.

15. [En ligne] : < http://www.dofus.com/fr >.

16. [En ligne] : < http://www.wakfu.com/fr/mmorpg >.

17. [En ligne] : < http://www.hordes.fr $>$.

18. [En ligne] : < http://mush.vg >.

19. [En ligne] $:<$ http://motion-twin.fr $>$. 


\section{PARTIE II}

\section{ACQUÉRIR, VALORISER}

1. CONSTITUER UNE COLLECTION DE JEUX : QUELS OBJECTIFS ? par Anthony Avila

$++++++++++++++++++++++++++++++++++++++++++++++++++++++++++++++++$

2. JOUER EN BIBLIOTHĖQUE, JOUETS EN BIBLIOTHĖQUE ?

par Caroline Makosza

$++++++++++++++++++++++++++++++++++++++++++++++++++++++++++++++++$

3. JOUER SÉRIEUSEMENT - POUR APPRENDRE ?

par Éric Sanchez

$++++++++++++++++++++++++++++++++++++++++++++++++++++++++++++++++$

4. CRÉER UN SERIOUS GAME EN BIBLIOTHĖQUe : L'EXEMPLE DE ESCOUADE B

par Thierry Robert

5. LES LUdo'MALLES DE LA MÉdiATHĖQUE DÉPARTEMENTALE DU PUY-DE-DÔME : UN OUTIL LÉGER POUR UN AUTRE REGARD SUR LES BIBLIOTHĖQUES

par Solenne Waszak 


\section{1 \\ CONSTITUER UNE COLLECTION DE JEUX : QUELS OBJECTIFS? \\ par \\ Anthony Avila}

L'offre en termes de jeux est variée et est, par bien des égards, plus complexe que le marché du livre ou de l'audiovisuel, par exemple. On joue avec de nombreux supports (jouets, jeux de société, livres, consoles de jeux, ordinateurs, tablettes, etc.) et pour de nombreuses raisons (s'amuser, apprendre, s'occuper, partager, etc.). Ainsi, constituer une offre de jeu en médiathèque ne peut pas être pris à la légère : il convient de définir des objectifs, de monter un véritable projet.

\section{LE PROJET}

Plusieurs questions se posent alors simultanément : qui constituera le fonds, pour quel public et avec quels moyens ? En découle alors le choix de l'offre qui sera proposée.

\section{QUI CONSTITUE LE FONDS ?}

Il existe des professionnels du jeu, les ludothécaires, qui font un travail similaire aux bibliothécaires mais autour du jeu sous toutes ses formes ${ }^{1}$. Pour constituer un fonds de jeu, il semble indispensable de passer soit par le recrutement d'un ludothécaire (tout comme on recrute des discothécaires pour s'occuper des fonds musicaux) ou au moins de former une partie du personnel à ce métier. Au minimum, une personne particulièrement sensible aux univers du jeu semble indispensable.

Cependant, si cela n'est pas possible, il est envisageable de suivre la méthode de la médiathèque Nelson Mandela d'Angers : un comité d'usagers a été constitué pour la création de leur fonds de jeux vidéo. Le personnel de la médiathèque a ainsi construit le projet et a choisi les titres qui seraient achetés en partenariat avec des usagers volontaires. Le comité

1. À l'exception du jeu vidéo, très rarement présent en ludothèque. 
continue de se réunir tous les trimestres depuis avril 2012 et est ouvert à tous, sans critère d’âge ou même de résidence.

\section{POUR QUEL PUBLIC ?}

Se pose en parallèle la question du public que l'on souhaite toucher avec les jeux. Les enfants viennent à l'esprit de la majorité des gens, et l'offre est effectivement très importante à leur intention. Il existe par ailleurs de très nombreux jeux de qualité dédiés aux adolescents et aux adultes qui méritent notre attention. En effet, si les plus jeunes sont un public très présent dans nos espaces, il peut être intéressant de développer en parallèle ce fonds pour toucher de nouveaux publics.

De même, la proposition qui sera faite entre jeu sur place et collection empruntable déterminera le type d'usagers et d'usages. Des adultes actifs préféreront pouvoir emprunter et ne seront pas disponibles plus d'une fois par mois pour une soirée jeux, tandis qu'il sera par exemple possible d'organiser des animations simples tous les samedis après-midi avec les enfants.

\section{AVEC QUELS MOYENS ?}

Enfin, les moyens disponibles, humains et financiers, définiront également le type de projet qui peut être mis en place. Avec peu de moyens, il vaudra mieux se concentrer sur une offre plus circonscrite mais efficace, plutôt qu'essayer de tout faire en petite quantité et de frustrer le public.

Le prix moyen d'un jeu de société pour les moins de huit ans se situe aux alentours de $15 €$, tandis que celui d'un jeu pour les plus de huit ans monte à près de $30 €$. Pour les jeux vidéo, les prix varient en général de 40 à $70 €$ pour un jeu neuf, auxquels il faut ajouter le coût des consoles de jeux, entre 200 et $500 €$.

Ainsi, il sera aisé, avec des fonds d'acquisition réduits, de monter un petit fond d'animation sur place, alors qu'une offre de prêt demandera plus de temps ou plus d'argent pour devenir viable. Il faut cependant prendre en compte la surcharge de travail en accueil et en animation que cela représente en plus des activités habituelles des agents.

Il s'agit donc d'être correctement informé et de définir des objectifs clairs en fonction de ces trois critères : quels agents (avec quelle formation), 
pour quels publics et avec quels moyens ? Pour cela, nous allons étudier un peu plus en profondeur les types de jeux existants et ce que l'on peut faire avec, comment les sélectionner et les acquérir, avec un focus particulier sur les jeux vidéo.

\section{UN FONDS DE JEUX « CLASSIQUES 》}

$++++++++++++++++++++++++++++++++++++++++++++++++++++++++++++++++++$

On pense souvent avant tout aux jeux de société, quand on imagine du jeu en bibliothèque ou même en ludothèque, mais ce n'est en fait qu'une des dimensions indispensables du jeu. Les ludothécaires ont mis en place un système de classification, ESAR ${ }^{2}$, pour exercice, symbolique, assemblage et règles.

\section{LE SYSTÈME DE CLASSIFICATION ESAR :}

\section{ENCADRÉ 4 GRANDES CATÉGORIES DE JEUX}

Les jeux d'exercice sont les jeux des tout-petits, qu'on peut aussi appeler jeux d'éveil. On y joue pour un résultat sensoriel fort et immédiat. Ils vont du trampoline aux jouets sonores en passant par les jeux de miroir ou de manipulation.

Les jeux symboliques sont ceux qui permettent de faire semblant. C'est autant le fait d'enfiler un costume que de jouer avec un baigneur ou avec des figurines et d'inventer des histoires. Pour les plus grands, ce sera par exemple les jeux de rôle sur table, qui incluent par ailleurs des mécanismes de jeux de règles.

Les jeux d'assemblage sont ceux qui permettent d'assembler ou de construire des choses. On pense évidemment aux Lego ${ }^{\circledR}$ ou aux Kapla, mais il en existe de nombreux autres. Ils ont la particularité d'être particulièrement transgénérationnels et de passionner de la même façon autant les adultes que les enfants.

Enfin, les jeux de règles sont ceux que l'on appelle en général jeux de société. On y suit un lot de règles précises communes aux différents joueurs. On y retrouve évidemment les jeux de plateau, mais aussi les jeux de cartes, de dés, ou même les sports quand ils sont pratiqués pour le plaisir.

2. Denise Garon, Rolande Filion et Robert Chiasson (collab.), Le système ESAR : guide d'analyse, de classification et d'organisation d'une collection de jeux et jouets, Montréal, Éd. ASTED ; Paris, Éditions du Cercle de la Librairie, 2002. 


\section{Jeux de société}

Si les jeux d'exercice, symboliques et d'assemblage sont relativement bien connus des gens - au moins des parents et des animateurs petite enfance - la variété de la production de jeux de règles en boîte reste largement méconnue, malgré un succès grandissant et une production annuelle toujours plus importante.

En effet, au-delà des classiques Monopoly ${ }^{\circledR}$, Cluedo ${ }^{\circledR}$ et autres Pictionnary ${ }^{\circledR}$, il y a depuis quelques années de nouveaux éditeurs et auteurs qui ont su faire preuve d'originalité et proposer de nouveaux concepts particulièrement efficaces, pour tous les âges.

Djeco est sans doute le meilleur éditeur pour le jeune public, avec des jeux solides et bien conçus, aux côtés de Haba, qui propose également de très beaux jeux en bois. Asmodée est un éditeur qui a su conquérir le grand public avec des jeux courts et intenses que l'on trouve désormais même dans certaines grandes surfaces. Mais il y en a d'autres encore, comme Days of Wonder, Edge, Iello, Filosofia, Queen Games, etc. Il est possible de s'informer sur l'actualité de ces jeux au travers de la presse papier $\left(\right.$ Plato $^{3}$ ) ou de sites web (Tric Trac ${ }^{4}$ ou La sélection du dimanche ${ }^{5}$ ). Un certain nombre de prix d'excellence sont distribués dans le monde, dont notamment l'As d'Or ${ }^{6}$ et le Spiel des Jahres ${ }^{7}$.

Ces quatre catégories permettent non seulement de catégoriser toutes les formes de jeu, mais forment aussi un tout indissociable d'une ludothèque équilibrée. Cependant, ce qu'il est possible de faire est dépendant de l'espace consacré au jeu.

3. Le dernier magazine dédié aux jeux de société encore publiée en France. [En ligne] : $<$ http:// www.plato-magazine.com >.

4. Actualité et base de données encyclopédique des jeux de société. [En ligne] : < http://www.trictrac.net >.

5. Blog essentiellement dédié aux jeux de société. [En ligne] : < http://www.laselectiondudimanche.com >.

6. Délivré chaque année lors du Festival international des jeux de Cannes (voir la liste des jeux primés). [En ligne] : < http://fr.wikipedia.org/wiki/As_d'or_Jeu_de_l'année >.

7. Délivré chaque année en Allemagne mais sans doute le prix majeur du milieu (voir la liste des jeux primés). [En ligne] : < http://fr.wikipedia.org/wiki/Spiel_des_Jahres >. 


\section{QUEL ESPACE DISPONIBLE ?}

En effet, le jeu est une pratique qui peut nécessiter un espace important, et peut empiéter sur les activités habituelles de la médiathèque, ne seraitce que par le bruit et le mouvement qui sont générés par l'activité.

La ludo-médiathèque de Fosses par exemple a mis en place un espace jeu central dans le bâtiment, avec notamment une grande place dédiée aux jeux d'exercice, symboliques et d'assemblage, libres d'accès. Cependant, cela se fait au détriment des autres collections, moins conséquentes, et surtout du calme global du lieu. Le jeu est néanmoins particulièrement bien mis en avant et rythme la vie du lieu.

La médiathèque de Sotteville-lès-Rouen a mis en place une offre de jeux, notamment de jeux de règles, sans pour autant prévoir un espace dédié. Les jeux sont en accès indirect au niveau d'un bureau d'accueil dédié, et les usagers peuvent les emprunter pour les emmener ou pour jouer sur place dans les espaces déjà existants.

D'autres médiathèques mettent en place des événements plus ponctuels dans un espace fermé, voire même à des horaires spécifiques, comme la médiathèque Blaise-Cendrars de Conflans-Sainte-Honorine qui organise régulièrement des animations jeux de rôle en soirée, par exemple.

L'espace qui pourra être mis à disposition pour le fonds définira largement ce qui peut effectivement être fait. Les pratiques ludiques, même dans les meilleures conditions, seront toujours plus bruyantes que les autres activités habituelles de la médiathèque. S’il est possible de dédier un espace fermé ou que le bruit ne pose pas de problème, tout est possible. Par contre, si l'aménagement des lieux ne peut pas être adapté au jeu, du prêt uniquement, voire de l'animation ponctuelle est à envisager.

\section{LES MODALITÉS D'ACQUISITIONS}

Pour effectuer les acquisitions, il faut respecter les règles des procédures de marchés publics. Celles-ci autorisent des démarches adaptées au-dessous de $15000 €$. Avec un budget annuel raisonnable, il est possible de faire une simple mise en concurrence : il faut contacter au moins trois fournisseurs et comparer leur offre (attention, la valeur du marché peut être cumulée sur plusieurs années, ainsi, en cas de marché reconductible sur trois ans à hauteur de $5000 €$ par an, il faut passer par un appel 
d'offres). Dans certains cas, il est possible pour des sommes plus importantes de passer par un marché à procédure adapté (MAPA) qui permet par exemple de limiter l'offre à des fournisseurs sélectionnés à l'avance, toujours trois au minimum, mais avec un acte d'engagement plus fort et plus sécurisé de la part du fournisseur.

La médiathèque d'Osny, par exemple, a mis en place des mises en concurrence à hauteur de $4000 €$ chaque année pendant trois ans pour constituer le fonds et envisage de dépenser environ $1000 €$ par an par la suite pour entretenir le fonds.

Il est possible de passer par certains grossistes habitués des marchés publics comme Wesco et Ogeo, essentiellement pour les jeux d'exercice, symboliques et d'assemblage et les jeux de règles pour les plus petits. Pour des jeux de société adulte et des jeux de rôle, il faudra passer par de plus petites boutiques en général, comme Ludik Bazar ${ }^{8}$ et Philibert ${ }^{9}$ qui répondent efficacement et rapidement aux demandes de devis par email. Il n'est pas nécessaire d'acquérir de droit particulier pour faire de l'animation sur place ou du prêt de jeux de société ou de jouets.

\section{UN FONDS DE JEUX VIDÉO}

Les jeux vidéo sont un support particulier de jeux qui naviguent la plupart du temps entre jeu de règles et jeu symbolique, bien qu'il existe également des jeux vidéo de construction virtuelle et même des jeux d'exercice. Ils ont un succès certain depuis quelques années et touchent une partie de plus en plus importante de la population, au point d'être devenus une des industries culturelles les plus importantes en France.

Les mêmes contraintes concernant l'espace sont valables pour les jeux classiques et les jeux vidéo : leur pratique est par nature assez bruyante, surtout en groupe, mais il est aussi possible de jouer plus silencieusement avec un casque audio. Là encore cependant, un espace dédié sera préférable pour faire de l'animation sur place.

8. [En ligne] : < http://www.ludikbazar.com >.

9. [En ligne] : < http://www.philibertnet.com $>$. 
Le choix des acquisitions se fera comme pour les autres jeux en fonction des agents formés, du public visé et des moyens disponibles, mais d'autres obligations entrent en jeu. Il existe en effet des protections sur certains jeux qui empêchent de les prêter, notamment sur les jeux sur ordinateur. En effet, le jeu est associé à un compte utilisateur à la première utilisation et ne peut pas être installé par un autre emprunteur par la suite. Il conviendra donc d'être vigilant.

Par ailleurs, comme pour les autres jeux, l'offre de jeux vidéo est riche et complexe et nécessite de s'informer activement. Des formations et des journées d'étude sont proposées régulièrement sur le sujet, mais il est possible de suivre également la presse (Jeuxvideomagazine.com ${ }^{10}$, Games ${ }^{11}$ ) ou certains sites web (jeuxvideo.com ${ }^{12}$, gamekult.com ${ }^{13}$, indius.org ${ }^{14}$ ) pour se tenir informé de l'actualité.

\section{LES MODALITÉS D'ACQUISITIONS}

Les mêmes règles de marchés publics s'appliquent aux jeux vidéo.

Le réseau des médiathèques de Cergy (trois équipements) a acquis environ $5000 €$ de jeux vidéo par an depuis trois ans pour faire de l'animation ponctuelle essentiellement, mais ils envisagent depuis peu de faire du prêt également. Il n'y a que des jeux et des consoles actuels.

La médiathèque d'Osny, dans le cadre de sa construction, a pu débloquer des fonds importants : $15000 €$ par an pendant trois ans ont été dépensés pour constituer le fonds, et il est envisagé de dépenser un peu plus de $2500 €$ par an par la suite pour entretenir la collection. Le fonds sera divisé entre fonds patrimonial (un tiers) et jeux actuels (deux tiers), sur console exclusivement. Des jeux sur ordinateur seront proposés également, mais uniquement sur place. Tout le reste sera proposé en prêt, jeux comme consoles de jeux. La particularité de ce fonds est qu'il a été pensé comme n'importe quelle autre acquisition de la médiathèque, avec autant d'achats de " classiques » (les premiers Mario, d'anciennes consoles, etc.) que d'actualité.

\footnotetext{
10. Magazine spécialisé classique, sur les « blockbuster » du jeu vidéo essentiellement.

11. Magazine plus pointu sur les artistes et auteurs du monde des jeux vidéo.

12. Le site web le plus consulté en France sur les jeux vidéo.

13. Site web d'actualité connu pour la sévérité de ses tests.

14. Site web d'actualité spécialisé dans les jeux indépendants.
} 
De manière générale, $5000 €$ permettent de monter un fonds représentatif de l'année pendant laquelle il est créé (y compris les consoles), mais le nombre de jeux sera un peu faible pour faire du prêt sans frustrer les usagers. C'est cependant un chiffre correct sur plusieurs années, pour constituer un fonds petit à petit.

Certains fournisseurs de médiathèques spécialisés dans l'audiovisuel - comme CVS, Colaco ou RDM ${ }^{15}$ - proposent dans leur catalogue du jeu vidéo avec droits de consultation uniquement, mais il semblerait que les droits ne soient pas vraiment négociés. Il n'est pas indispensable donc de passer par ces prestataires qui par ailleurs gonflent les prix. L'idéal est de passer par des boutiques spécialisées. Les FNAC sont en général en mesure de travailler efficacement avec des collectivités, ainsi que certaines boutiques, comme GameSpirit ${ }^{16}$. Il est possible de se tourner vers une boutique locale par ailleurs. Les Micromania sont théoriquement en mesure de fournir une collectivité, mais il faudra passer par le siège de l'entreprise pour certaines démarches administratives et par le magasin pour le devis.

Il peut être intéressant également d'installer une borne d'arcade* dans les espaces publics. Il existe des fournisseurs ${ }^{17}$ et des formats de plus en plus nombreux, certains proposant des machines avec plusieurs milliers de jeux inclus pour du jeu sur place. Il faut compter de 1200 à $3500 €$ selon les modèles. L'important est de construire un projet adapté à la situation de la médiathèque. Il faut bien prendre en compte les espaces, et les moyens - pour savoir ce qu'il est possible de faire - mais aussi faire des choix quant au public que l'on souhaite attirer avec ce genre d'offre. Il existe autant de façons de jouer que de joueurs, et une offre à l'image de cette variété. Vouloir tout faire peut autant diluer l'offre que trop se concentrer sur certaines choses risque d'exclure certaines pratiques. Il n'y a cependant pas de recette parfaite, et c'est à chaque équipement de trouver l'équilibre qui lui convient.

15. CVS : < http://www.cvs-mediatheques.com/ > ; Colaco : < http://www.colaco.fr/ > ; RDM vidéo : < http:// www.rdm-video.fr/ $>$.

16. [En ligne] : < http://www.gamespirit.fr >.

17. The Arcade Company pour des mini-bornes. [En ligne] : < http://www.the-arcade-company. com >, Neo Legend, un des plus anciens acteurs du marché en France, matériel de très bonne qualité. [En ligne] : < http://www.neo-legend.com >, Neo Arcadia, machines japonaises de série. [En ligne] : < http://arcade.am >, Game Spirit est également revendeur. [En ligne] : < http:// www.gamespirit.fr >, etc. 


\begin{tabular}{|l|l|}
\multicolumn{2}{|c|}{2} \\
\hline JOUER EN BIBLIOTHĖQUE, & par \\
JOUETS EN BIBLIOTHĖQUE ? & Caroline \\
\hline
\end{tabular}

\section{POURQUOI DES JOUETS ?}

Si l'on veut proposer du jouer en bibliothèque, alors il faut proposer du jouet. C'est le jouet et surtout ce que l'enfant en fait, qui construit toute la culture de l'enfance ${ }^{1}$. Les bibliothèques proposent des albums, des bandes dessinées et des dessins animés parce qu'elles ont compris que c'était ce que lisent et regardent les enfants, et que ces pratiques contribuent à la construction de leur culture. Ainsi, les enfants lisent des albums et des bandes dessinées, regardent des dessins animés et jouent avec des jouets. Ce sont eux qui construisent notre offre.

À travers le jeu symbolique (les jouets d'imitation, type dînettes, poupées, déguisements, circuits, figurines...), l'enfant va pouvoir rejouer son quotidien, s'approprier les codes sociaux utiles à la vie en commun, ou bien inventer, seul ou avec ses amis, des mondes où tout est possible, sans risque, sans jugement et sans peur de l'échec. Et souvenons-nous, finalement il suffit de cela : se rappeler ses propres expériences de jeu et les sensations qu'elles nous ont procurées, des histoires que l'on s'est inventées, des sentiments que l'on a apprivoisés, des heures qui passaient comme des minutes, pour finir, s'il en est besoin, d'être convaincu par l'intérêt du jeu symbolique.

\section{LES ENJEUX POUR UNE MÉDIATHĖQUE}

Votre première tâche, bien avant d'aller dévaliser les magasins de jouets, sera de convaincre votre hiérarchie, les élus, du bien-fondé de mettre des jouets à disposition du public de la bibliothèque. En effet, le jouet

1. Pour ces aspects culturels du jouet, voir Gilles Brougère, Jouets et compagnie, Paris, Stock, 2003 (coll. Un ordre d'idées) et Gilles Brougère (dir.), La ronde des jeux et des jouets : Harry, Pikachu, Superman et les autres, Paris, Autrement, 2008 (coll. Mutations), qui aborde notamment les notions de "produits dérivés » également au travers de la littérature enfantine. 
souffre d'une image assez négative dans la société en général et dans les milieux culturels tout particulièrement. Pas très écologique, avec une production fortement délocalisée, le jouet est également très marqueté, attaché à l'image de la consommation de masse. Le jeu de règle, ou le jouet " éducatif », est une manière pour les adultes de prolonger les apprentissages reçus à l'école alors que le jouet permettant à l'enfant de se créer ses propres histoires est entièrement déconnecté de l'aspect éducatif scolaire. Le jeu de l'enfant échappe alors quasiment complètement à la compréhension et au contrôle de l'adulte. Il est pourtant primordial dans la construction affective et cognitive de l'enfant pour sa vie d'enfant et pour sa vie d'adulte. Il constitue une " première exploration vers d'autres mondes possibles $»^{2}$. Ce sont ces concepts qu'il faudra maîtriser afin de faire comprendre à vos élus, mais aussi à une partie des publics, qui ne manquera pas de marquer son étonnement, l'intérêt du jeu symbolique. À la médiathèque, il nous a fallu convaincre notre hiérarchie, nos élus, les parents et le monde enseignant (du moins en partie) avant d'aménager des espaces pour les jouets à la ludo-médiathèque. Cela implique donc une bonne connaissance des enjeux du jeu symbolique qui doit être partagée par l'ensemble de l'équipe. Et il faut également se préparer à recommencer sans cesse ce travail d'explication auprès des nouveaux visiteurs mais aussi au gré des changements des équipes municipales ou intercommunales.

\section{QUELS AMÉNAGEMENTS POUR LES ESPACES JEUX ?}

Inutile de dépenser des sommes folles au départ, cependant, si vous dépensez peu, dépensez bien. Préférez toujours la qualité à la quantité, vous consoliderez votre offre au fur et à mesure des années.

Dans tous les cas, que vous disposiez de beaucoup ou de peu de moyens, pensez tous vos espaces avant d'acheter les objets.

Il existe assez peu d'ouvrages ou de formations sur l'aménagement des espaces de jeux, ou sur le jeu en général et encore moins appliqués aux

2. Paul L. Harris, L'imagination chez l'enfant, son rôle crucial dans le développement cognitif et affectif, Paris, Retz, 2007 (coll. Forum éducation culture). 
bibliothèques. Nous ne pouvons que vous conseiller de contacter l'Association des ludothèques françaises $(\mathrm{ALF})^{3}$ ou l'antenne qui correspond à votre région. Le livre Des espaces pour jouer ${ }^{4}$ peut aussi être une aide non négligeable même s'il s'adresse plus spécifiquement aux ludothèques. Enfin, l'association Les enfants du jeu ${ }^{5}$ à Saint-Denis (93) peut vous aider à porter un projet d'aménagement ainsi qu'une réflexion plus large sur la place du jeu dans les accueils publics. D’une manière générale, les bibliothèques publiques ont beaucoup à apprendre des ludothèques. Proposer du jeu en bibliothèque ne saurait se faire sans se rapprocher d'abord des professionnels du jeu et notamment des ludothécaires. Les deux professions ne peuvent que s'enrichir l'une l'autre et auront grand intérêt, dans certains cas, à fusionner pour proposer au public un lieu riche et une offre large, diversifiée et de qualité.

Nadège Haberbusch ${ }^{6}$ propose quelques principes liés à l'aménagement d'espaces de jeux symboliques : «L'autorisation à jouer doit être inscrite dans l'aménagement. Le libre accès aux jouets, la composition des espaces ainsi que leurs agencements doivent permettre aux enfants de pouvoir détourner les objets, d'être créatifs et inventifs. La circulation des enfants doit aussi être prévue car elle est souvent génératrice de conflits. Il est intéressant de leur offrir un panel varié de jouets évoquant la réalité et des mondes imaginaires. Évidemment, un espace rangé, soigné et esthétique valorise l'activité de l'enfant. Enfin, si un espace pertinemment aménagé, valorisé par la présence adaptée et réfléchie d'un professionnel, favorise la mise en jeu de l'enfant, il ne faut pas que les règles de fonctionnement du lieu constituent un frein au jeu. ${ }^{7}$

3. ALF : $<$ http://www.alf-ludotheques.org/ >.

4. Odile Périno, Des espaces pour jouer : pourquoi les concevoir ? Comment les aménager ?, nouvelle édition entièrement revue et augmentée, Toulouse, Erès, 2014 (coll. Enfance et parentalité).

5. < http://www.les-enfants-du-jeu.com/ >.

6. Je profite de ces lignes pour humblement la remercier pour le rôle extrêmement important qu'elle a joué dans les orientations de notre projet.

7. Nadège Haberbusch, « Le jeu symbolique en ludothèque », Ludo, décembre 2010, n³2, pp. 10-13. 


\section{LES 5 ESPACES JEUX DE LA LUDO-MÉdIATHĖQUE}

\section{ENCADRÉ DE FOSSES}

À la ludo-médiathèque, nous avons organisé les jouets à l'intérieur de cinq espaces plus ou moins déclinés dans la plupart des ludothèques :

- les jeux de règle : le sujet est largement développé dans la contribution « Jouer pour jouer » (p. 110) ;

-petite enfance : achetez des jouets adaptés aux tout-petits mais n'en proposez pas de trop nombreux. Évitez les jouets pseudo-éducatifs et/ou difficiles à manipuler et les jouets à piles qui font du bruit pour rien. Les jouets proposés aux tout-petits sont en général de piètre qualité ludique et matérielle. Trop difficiles à manipuler (ne tenez pas trop compte des âges mentionnés sur les boîtes) ou sans intérêt pour les petits. Essayez-les avant et allez au plus simple : boîtes à formes, hochets, peluches, objets à empiler, secouer, manipuler... Aménagez un espace protégé avec des claustras par exemple et des tapis facilement lavables. Pensez à proposer des assises pour les adultes accompagnants ;

- jeux symboliques : des jouets de "faire semblant ", dînettes, poupées, nurserie, marchands, restaurants... Beaucoup d'univers sont possibles, il en faut au moins deux pour avoir des interactions intéressantes. Proposez également des déguisements et accessoires. Observez soigneusement l'utilisation que feront les enfants de cet espace, cela vous permettra de le réadapter au mieux à la pratique ;

-jeux de construction : ils s'adressent à tous les âges. Prévoyez un peu de place et des jeux type Kapla, briques de construction, Lego®, Lego Technic ${ }^{\circledR}$ et autres Duplo. Mettez aussi des modèles à disposition et/ou des photos de constructions faites par vous ou vos visiteurs. Si vous avez des vitrines, vous pouvez aussi exposer certaines œuvres ! L'espace de jeux de construction peut se trouver près des espaces symboliques et/ou figurines, le matériel pouvant servir à fabriquer des objets ou des décors qui manquent aux enfants pour enrichir leurs scénarios ;

-jeux de figurines : proposez plusieurs univers différents : château fort, ferme, dinosaures, voitures et trains, pompiers, Far-West, maisons de poupées... faites tout de même attention à ne pas investir des sommes folles dans des figurines hors de prix, les enfants ont parfois tendance à oublier qu'elles ont sauté dans leurs poches... N'oubliez pas les décors! Vous pouvez proposer ici des tapis et/ ou des petits podiums pour les mises en scène des histoires. 
En conclusion, allez jusqu'au bout de votre souhait de proposer du jeu aux enfants. Prenez contact avec des ludothèques ou d'autres professionnels du jeu proches de vous et lancez-vous. Beaucoup de projets différents, même à très petit budget, peuvent voir le jour en respectant et en valorisant toutes les formes de jeu. Il ne vous reste qu'à trouver celui qui convient à votre établissement et à votre public. 


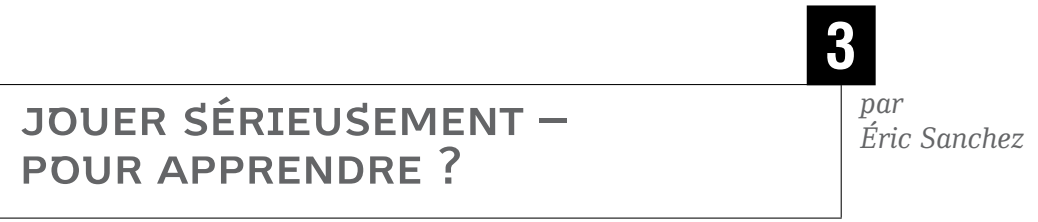

L'idée que le jeu puisse avoir des vertus éducatives n'est pas nouvelle et le mot latin ludus, qui désigne simultanément le jeu et le travail scolaire, vient nous rappeler que l'opposition entre ces deux types d'activités ne va pas forcément de soi. Devenues aujourd'hui l'une des premières industries culturelles en termes de chiffres d'affaires, les jeux vidéo s'invitent de nombreux secteurs de l'activité humaine dont ceux de l'enseignement et de la formation. Déjà en 1989, le philosophe Jacques Henriot écrivait : "Le monde où je vis est un monde où il est de plus en plus question de jeu : non seulement parce qu'il semble que l'on joue chaque jour davantage, mais surtout parce que l'idée même du jeu s'applique constamment à de nouvelles situations, à des formes de conduite et de pensée auxquelles il eût paru, récemment encore, inconvenant de l'appliquer. ${ }^{1}$ Ainsi, des jeux, qualifiés de sérieux, bénéficiant pour leur conception d'opportunités nouvelles offertes par le numérique, sont aujourd'hui conçus pour des visées éducatives.

Dans cette contribution, nous aborderons la question de la modalité des apprentissages dans le cadre de formations qui intègrent l'usage de jeux sérieux. Comment apprend-on en jouant avec de tels jeux ? Quels sont les éléments à prendre en compte pour leur usage ? En premier lieu, nous discuterons des différentes significations du terme « jeu » lui-même pour montrer que son caractère polysémique est source de confusion quand on lui adjoint l'adjectif " sérieux ». Une seconde partie sera consacrée au pouvoir motivationnel des jeux qui est l'argument le plus souvent mis en avant quand il est question de promouvoir leur usage. Enfin, une troisième partie nous conduira à discuter l'idée d'apprentissage expérientiel avec les jeux et la question de leur intégration dans les dispositifs de formation.

1. Jacques Henriot, Sous la couleur de jouer : la métaphore ludique, Paris, José Corti, 1989, p. 27. 


\section{JEU SÉRIEUX, EXÉGĖSE D'UN TERME POLYSÉMIQUE}

L'expression jeu sérieux recouvre des réalités parfois bien différentes. En effet, quel point commun y a-t-il entre un jeu vidéo tel que Zombie Division $^{2}$, qui consiste, pour des enfants de 6-7 ans, à tuer des zombies pour apprendre la division, Tamagocours ${ }^{3}$, un tamagotchi ${ }^{4}$ multi-joueur en ligne destiné à la formation de futurs enseignants aux règles juridiques qui encadrent l'usage des ressources numériques dans un contexte pédagogique, Mac Donald's ${ }^{5}$, un jeu en ligne conçu par le collectif italien Molleindustria pour dénoncer les méfaits du restaurateur éponyme ou Fort Mc Money ${ }^{6}$, un "game-documentaire »* qui permet au spectateur-joueur de prendre conscience des enjeux liés à l'exploitation des schistes bitumineux et d'expérimenter, par lui-même, les conséquences économiques, environnementales ou sociétales de ses propres choix politiques ? L'oxymore vient ici désigner un matériel ludique qui permet de combiner la frivolité de l'usage attendu et l'importance de l'enjeu éducatif. Il renvoie à des terminaux variés (ordinateurs fixes ou dispositifs tactiles et mobiles connectés ou pas à Internet), des formes ludiques diverses (jeux personnels ou collaboratifs) et des apprentissages de nature très différente.

Pourtant, les travaux qui se sont intéressés au jeu, à son rôle dans le développement de l'enfant et le processus d'apprentissage mettent l'accent sur la situation vécue ou les interactions mises en place plutôt que sur le matériel ludique, les artefacts qui sont utilisés. Ainsi, pour Jean Piaget et Bärbel Inhelder, le jeu apparaît comme un « secteur d'activités dont la motivation [n'est pas] l'adaptation au réel, mais au contraire l'assimilation du réel au moi, sans contraintes ni sanctions » ${ }^{7}$. Pour Donald Woods

2. Matthew Peter Jacob Habgood, The effective integration of digital games and learning content, $\mathrm{PhD}$ Thesis PhD, University of Nottingham, 2007.

3. Éric Sanchez, "Tamagocours, un jeu numérique sur les règles qui encadrent les usages des ressources numériques ", Actes du VII e colloque Questions de pédagogies dans l'enseignement supérieur, Sherbrooke, Québec, Canada, 2013, pp. 528-536. [En ligne] : < http://www.colloquepedagogie.org/?q=node/433 >.

4. Un tamagotchi est un jeu de simulation persistant qui consiste à prendre soin d'un petit animal de compagnie virtuel.

5. < www.mcvideogame.com >.

6. < www.fortmcmoney.com >.

7. Jean Piaget et Bärbel Inhelder, La psychologie de l'enfant, $3^{\mathrm{e}}$ éd., Paris, PUF, 2012 (coll. Quadrige). 
Winnicott ${ }^{8}$, lorsque l'enfant joue, il entre dans une aire intermédiaire où la réalité intervient non plus comme une contrainte, mais se voit remodelée en fonction de ses besoins internes. Cette aire intermédiaire constitue un espace de liberté qui autorise l'expérimentation. De ce fait, le jeu, en tant que situation vécue, contient en germe le développement de l'individu qui s'y construit en mettant en œuvre sa créativité. C'est ainsi que, pour désigner les jeux, Lev Vygotsky ${ }^{9}$ utilise le terme de « situations », celles-ci permettant le développement de l'enfant.

En français, le mot « jeu » est donc ambigu tant il désigne simultanément la structure ludique, le game, un système de règles que le joueur décide de respecter et le playing, la pratique de ce joueur, dans le cadre d'une situation ludique ou play. Il faut également relever que le jeu dépend avant tout d'une décision du joueur qui accepte, ou pas, de jouer et nous rejoignons Jacques Henriot quand il écrit que « le jeu n'est pas dans la chose mais dans l'usage qu'on en fait ${ }^{10}$. Ainsi, plutôt que l'artefact numérique, le serious game, il nous semble important de considérer les interactions qui émergent de la situation et le jeu numérique épistémique ${ }^{11}$, en tant que situation ayant des visées éducatives (ou épistémiques), élaborée avec des technologies numériques.

Les caractéristiques de cette situation sont qu'il s'agit d'une activité de second degré, d'une part en tant que simulation ou métaphore d'un domaine de référence (division mathématique, gestion d'une entreprise de restauration rapide, usage pédagogique de ressources numériques ou exploitation de schistes bitumineux pour reprendre les exemples cités plus haut) et, d'autre part, parce que « Jouer c'est savoir qu'on joue. »12. Ainsi, le jeu Mac Donald's conduit le joueur à polluer, surexploiter l'environnement, exproprier ses habitants et maltraiter ses employés afin de gagner des

8. Donald Woods Winnicot, Jeu et réalité : l'espace potentiel, Paris, Gallimard, 2002 (coll. Folio. Essais ; 398).

9. Lev Vygotski, "Play and Its Role in the Mental Development of the Child", Voprosy psykhologii, $1966, n^{\circ} 6$.

10. Jacques Henriot, Le jeu, Paris, PUF, 1969 (coll. Initiation philosophique ; 86).

11. Éric Sanchez, Caroline Jouneau Sion, Ludovic Delorme, Shawn Young, Christelle Lison and Nicolas Kramar, "Fostering Epistemic Interactions with a Digital Game. A Case Study about Sustainable Developments for Secondary Education", Paper presented at the IOSTE XV International Symposium, La Medina - Yasmine Hammamet, Tunisia, 2012.

12. Jacques Henriot, Sous la couleur de jouer : la métaphore ludique, op. cit., p. 147. 
points mais la frivolité du jeu autorise une certaine distanciation sur laquelle repose le message que les concepteurs du jeu veulent transmettre. Une seconde caractéristique est la liberté offerte au joueur de prendre des décisions mais c'est une liberté encadrée par des règles arbitraires auxquelles le joueur accepte de se soumettre. Ces caractéristiques fondamentales du jeu lui confèrent un certain intérêt pédagogique, d'une part, en tant que dispositif techno-pédagogique qui facilite l'engagement du joueur dans une démarche d'apprentissage et, d'autre part, en tant qu'espace de réflexivité qui permet au joueur-apprenant d'expérimenter sa manière de penser et d'agir. Ce sont ces deux dimensions que nous développons ci-après.

\section{JEU ET MOTIVATION}

Placere et docere, plaire et instruire, ce principe qu'on attribue au poète Horace (65-8 av. J.-C.), est celui le plus souvent convoqué lorsqu'il s'agit de justifier de l'utilisation de jeux numériques pour des visées éducatives. Ainsi, depuis Érasme (1469-1536), le jeu est généralement considéré comme une ruse pédagogique permettant d'augmenter la palatabilité d'un contenu éducatif a priori considéré comme peu attractif et non pas comme ayant une valeur éducative intrinsèque ${ }^{13}$. Les contradicteurs de ce type de pédagogie ont donc beau jeu de dénoncer cette chocolate-covered broccoli approach ${ }^{14}$. Sous la saveur chocolatée du jeu subsiste l'amertume du savoir à apprendre.

\section{SENTIMENT DE COMPÉTENCE, RELATIONS SOCIALES ET AUTONOMIE}

Pourtant, considérée comme un élément clef du processus d'apprentissage, la motivation est une dimension souvent abordée par la recherche en éducation et son importance largement démontrée. Richard Ryan et

13. Gilles Brougère, Jeu et éducation, Paris, L'Harmattan, 1995 (coll. Éducation et formation. Série. Références).

14. Amy Bruckman, “Can educational be fun?", Paper presented at the Game Developers Conference'99, San Jose, California, 1999. 
Edward Deci ${ }^{15}$ proposent un cadre pour penser cette question en contexte éducatif. Selon ces auteurs, la motivation résulte de la volonté de satisfaire des besoins fondamentaux et universels qui comprennent le sentiment de compétence, les relations sociales et l'autonomie.

Le sentiment de compétence est lié à la clarté des objectifs à atteindre dans un jeu. Ce sentiment dépend de la capacité du joueur à se représenter l'issue du jeu en termes de réalisations. Avec Zombie Division, l'enfant comprend très vite l'enjeu (" diviser » le plus grand nombre possible de zombies) et les conditions pour l'atteindre, c'est-à-dire la nécessité de choisir une arme adéquate (un diviseur du numéro indiqué sur le dossard que porte le zombie). Mais le sentiment de compétence dépend également du niveau de difficulté du défi à relever qui doit donc être adapté aux capacités du joueur. Cela peut se traduire par différents niveaux de jeu dont la complexité augmente au fur et à mesure de la progression vers l'enjeu. Ainsi, pour Tamagocours, au premier niveau de jeu, la plupart des « ressources " mises à disposition sont licites et donc digestes pour le tamagotchi qu'elles doivent permettre de " nourrir ». Ce premier niveau est donc difficilement perdable et il est moins destiné à l'apprentissage des règles juridiques que du gameplay*. Ce faisant, il permet au joueur de comprendre que l'issue du jeu dépendra de ses choix et l'aide à fonder ses décisions et actions sur une analyse du domaine que le jeu vise à enseigner. Les autres niveaux de jeu intègrent progressivement les règles du droit d'auteur et le jeu se complexifie.

Lorsque ces conditions sont remplies, le joueur peut alors accepter de prendre en charge le défi. Le cas échéant, les décisions qu'il prend sont motivées par la compréhension qu'il a de la situation plutôt que par les attentes de l'enseignant. Un défi motivant peut être également un élément important pour renforcer le sentiment de compétence. Le caractère motivant d'un défi dépend de facteurs intrinsèques au problème à résoudre (tels que sa nouveauté ou les conséquences de sa résolution) tous en lien avec les attentes du joueur qui pourra être motivé par tel ou tel aspect spécifique.

15. Richard M. Ryan and Edward L. Deci, "Self-Determination Theory and the Facilitation of Intrinsinc Motivation, Social Development, and Well Being", American Psychologist, 2000, n 55, pp. 68-78. 
La motivation est également en lien avec les relations sociales qui s'établissent du fait du jeu. Elle est liée au fait qu'un jeu, en tant que " méritocratie ${ }^{16}$, comprend des éléments de compétition. Cela se traduit par l'affichage de score, de tableaux d'honneur ou l'obtention de badges. Néanmoins, le succès dans un jeu peut également découler de la capacité à collaborer avec d'autres ou résulter d'une combinaison entre ces deux éléments. Ainsi, pour Tamagocours, il s'agit de jouer contre le jeu, en " nourrissant » correctement le tamagotchi, contre les autres équipes, en réalisant le meilleur score, mais aussi avec ses coéquipiers pour échanger et établir, collectivement, les stratégies gagnantes.

Enfin, la motivation du joueur, son niveau d'engagement, dépendent également de l'autonomie qui lui est offerte. L'autonomie comprend, d'une part, la liberté de faire des choix et de prendre ses propres décisions quant à la stratégie à adopter. La plupart du temps, il est nécessaire que différentes stratégies soient gagnantes. L'autonomie dépend également des conséquences de ses actions dans le jeu car ce sont ces rétroactions qui lui permettent de juger de la pertinence de ses décisions. C'est ce point que nous développons dans la partie suivante.

\section{LE JEU, ESPACE DE RÉFLEXIVITÉ}

L'idée d'apprentissage expérientiel est très prégnante dans la littérature consacrée au jeu. Ainsi, Jacques Henriot relève : "Si le jeu peut être un apprentissage de la vie, c'est parce qu'il met en mouvement des ressorts du même ordre que ceux auxquels font appel les activités concrètes ou réelles que l'on rassemble sous le nom un peu vague de vie. ${ }^{17}$ Pour John Dewey ${ }^{18}$, à qui l'on doit d'avoir développé l'idée d'apprentissage expérientiel, le problème posé par les méthodes traditionnelles d'éducation de son époque, provient du fait que ces méthodes se concentrent sur la prise en compte des facteurs externes qui influencent l'expérience du sujet apprenant mais négligent les facteurs internes qui déterminent cette

16. Douglas Thomas and John Seely Brown, A New Culture of Learning. Cultivating the Imagination for a Word in Constant Change, LaVergne, Createspace, 2011.

17. Jacques Henriot, Sous la couleur de jouer : la métaphore ludique, op. cit.

18. John Dewey, Experience and Education, New York, Free Press, 1997. 
expérience. À l'opposé, les jeux permettent, quant à eux, de vivre ce que Bernard Stiegler ${ }^{19}$ nomme, pour d'autres contextes, en référence à Socrate et au dialogue du Ménon, une expérience anamnésique, dans le sens où le savoir n'est pas extérieur au joueur mais en lui. À travers le jeu qui permet découvertes et inventions, des relations entre des éléments jusqu'alors isolés sont identifiées, des relations causales nouvelles sont mises à jour et de nouveaux problèmes sont construits. C'est ainsi une véritable " expérience heuristique rationnelle $»^{20}$ qui est vécue. Le jeu Fort Mc Money propose ainsi au joueur, de découvrir, au gré de ses explorations, la complexité des éléments sur lesquels doit se fonder la décision de développer, ou pas, l'exploitation des schistes bitumineux.

Cet apprentissage expérientiel provient donc du fait que les jeux conduisent le joueur à s'engager dans des investigations qui lui permettent de vivre une expérience originale au cours de laquelle il sera amené, comme dans d'autres secteurs de l'activité humaine, à apprendre de ses découvertes et à leur donner du sens. Le terme sérendipité repris de l'anglais serendipity, qui est souvent utilisé, vient exprimer le fait que, dans un jeu, le joueur apprend en faisant des découvertes inattendues lors de ses explorations. Ainsi, les arguments en faveur de l'usage de jeux en contexte éducatif sont en partie fondés sur l'idée piagétienne que l'apprentissage a une composante adaptative et que « les connaissances se manifestent essentiellement comme des instruments de contrôle des situations $»^{21}$. La connaissance est problématique et « un sujet manifeste ses connaissances dans ses interactions avec un milieu didactique selon les "règles" ou dans le cadre d'une situation ${ }^{22}$. Le concept de milieu didactique énoncé par Guy Brousseau est central pour comprendre comment jouer peut conduire à apprendre. Il s'agit du système avec lequel l'élève interagit. Si le milieu didactique - ici le jeu-game - réagit aux actions que l'élève exerce sur lui, ce dernier peut en tenir compte pour ses propres actions. L’apprentissage dépend, en

19. Denis Kambouchner, Philippe Meirieu et Bernard Stiegler, L'école, le numérique et la société qui vient, Paris, Éditions Mille et une nuits, 2012 (coll. Les petits libres ; 80).

20. Denis Kambouchner, Philippe Meirieu et Bernard Stiegler, L'école, le numérique et la société qui vient, ibid.

21. Guy Brousseau, La théorie des situations didactiques : cours donné lors de l'attribution à Guy Brousseau du titre de Docteur Honoris Causa de l'université de Montréal, 1997.

22. Ibid. 
conséquence, de la correction des actions effectuées et de l'anticipation de leurs effets. L'apprentissage est alors considéré comme un processus qui conduit l'apprenant à élaborer des connaissances en s'affrontant à un système - un jeu-game - qui lui permet, de manière continue, d'éprouver sa façon de penser et d'agir. Mais il ne s'agit pas d'un simple ping-pong béhavioriste qui conduirait à ce que le joueur-apprenant modifie ses actions en fonction des rétroactions selon un modèle stimulus/réponse mais plutôt la construction d'un espace de réflexivité au sein duquel il est placé en position d'analyser les conséquences de ses choix.

\section{DES CONDITIONS POUR L'APPRENTISSAGE}

Par ailleurs, un jeu-play est une situation où la connaissance du sujet se manifeste par des décisions et des actions régulières et efficaces sur un jeu-game sans qu'il soit nécessaire, pour l'avancée dans le jeu, que le joueur explicite les connaissances qu'il mobilise. Ces décisions et actions sont alors légitimées par la logique interne de la situation de jeu plutôt que par les attentes de l'enseignant ${ }^{23}$. Une telle situation peut donc fonctionner sans l'intervention de l'enseignant au niveau des connaissances. Cette dimension est souvent relevée dans la littérature consacrée aux jeux sérieux. L'utilisation d'un jeu permet à l'enseignant d'avancer masqué en cachant ses intentions didactiques.

Un jeu épistémique numérique peut être modélisé sous la forme d'un jeu-play, au cours duquel des interactions se mettent en place entre au moins un joueur-apprenant et un jeu-game qui constitue un dispositif d'évaluation formatif. Cette manière de modéliser l'apprentissage dans le cadre de jeux appelle deux remarques. En premier lieu, il faut relever que les connaissances qui sont développées dans une telle structure sont des connaissances procédurales qui sont caractérisées par le fait qu'elles sont contextualisées et implicites. Cela soulève l'importance de la phase de débriefing que connaissent bien tous les éducateurs qui ont intégré le jeu à leur arsenal pédagogique. Quitter le jeu permet de décontextualiser les connaissances qui changent alors de nature : instruments permettant la résolution d'un problème spécifique, elles acquièrent, du fait de leur 
institutionnalisation, une validité et un statut d'universalité qui rend possible leur utilisation dans d'autres contextes et permet le transfert des apprentissages. Par conséquent, on peut dire qu'on n'apprend du jeu qu'en quittant le jeu. La seconde remarque a trait à la nature des interactions que permet le jeu. L'usage d'un jeu-game pour des visées éducatives implique que ces interactions aient un haut niveau d'authenticité. Les données de la recherche suggèrent que les apprentissages basés sur le jeu, leur transfert, dépendent du degré de recouvrement entre le jeu-playing et les tâches de la situation de référence ${ }^{24}$. Par ailleurs, l'apprentissage dépend du fait que la connaissance visée soit optimale pour résoudre le défi proposé par le jeu. On utilise alors l'adjectif « intrinsèque » pour qualifier des jeux pour lesquels les connaissances mobilisées pour avancer dans le jeu sont également les connaissances visées par le dispositif de formation qui intègre le jeu.

\section{PERSPECTIVES POUR UNE PÉdAGOGIE ALTERNATIVE}

On peut relever une extension du domaine du jeu, qui concerne aujourd'hui, du fait de nouvelles opportunités offertes par le numérique, les domaines de l'éducation et de la formation. En effet, dans un contexte marqué par la nécessité de développer l'attractivité des formations, d'accueillir un nouveau public d'apprenants et de concevoir des dispositifs d'apprentissage qui permettent le développement de compétences plutôt que de savoirs strictement disciplinaires, les jeux épistémiques numériques apparaissent comme une pédagogie alternative aux approches traditionnelles. Nous utilisons à dessein l'expression « jeu épistémique numérique » plutôt que " jeu sérieux » car une rupture avec l'approche serious game, entendue comme l'usage de jeux vidéo dédiés à l'éducation/ formation, nous semble nécessaire. Cette rupture qui conduit à considérer le jeu-play plutôt que le jeu-game, permet de souligner que l'intérêt éducatif d'un jeu-game dépend moins de ses propriétés intrinsèques que de la manière dont l'enseignant ou le formateur l'intégreront dans

24. Sigmund Tobias and John Dexter Fletcher, "Reflections on 'A Review of Trends in Serious Gaming'”, Review of Educational Research, 2012, Vol. 82, n 2, pp. 233-237. 
leurs dispositifs de formation. Elle permet également de souligner que le domaine du jeu numérique ne se limite pas au jeu vidéo, mais qu'il peut concerner d'autres artefacts que l'ordinateur, mobiles, tactiles, combinés avec des éléments réels pour constituer des univers en réalité mixte, pour concevoir des situations d'apprentissage au sein desquelles l'apprenant pourra exercer sa créativité et s'entraîner à résoudre des problèmes complexes, non déterministes, tels les défis qui se présentent désormais aux citoyens du $\mathrm{XxI}^{\mathrm{e}}$ siècle. 


\section{CRÉER UN SERIOUS GAME EN BIBLIOTHĖQUE : L'EXEMPLE DE ESCOUADE B \\ par \\ Thierry Robert}

\section{4}

En septembre 2013, les bibliothèques de Montréal mettaient en ligne le jeu Escouade $B^{1}$, un jeu sérieux pour aider les jeunes de 9 à 12 ans dans l'apprentissage de l'information sur le Web. La mise en œuvre de ce projet dura plus de deux ans et fut développée en interne où une équipe de bibliothécaires joua les rôles de concepteurs, programmeurs, développeurs et animateurs. Depuis son lancement, plus de 10000 personnes ont joué à Escouade $B$ et le jeu connaît, encore maintenant, un vif succès à travers le programme d'animation Bibliothèques à la rescousse ${ }^{2}$. Les animateurs du programme ont maintes fois mentionné la plus-value de ce support pour l'enseignement et la transmission du savoir dans leur animation.

Ce projet numérique répondait à une demande spécifique du programme Bibliothèques à la rescousse qui désirait enseigner l'analyse de l'information sur le Web. En prenant en considération que cet apprentissage était particulièrement difficile sans support informatique et que les postes informatiques étaient limités en bibliothèque, la création d'un jeu semblait un support plus intéressant. La demande de départ était de concevoir un portail composé de cinq jeux d'apprentissage sur les compétences informationnelles (les étapes d'une recherche, la classification documentaire, la recherche en ligne, les ouvrages de référence et le rôle des bibliothèques) pour les jeunes de 9 à 12 ans ; mais devant la complexité de la conception et du développement des jeux, nous avons décidé de prioriser un seul projet : Escouade $B$.

Nous reviendrons sur les défis et les réussites de ce projet dans une perspective bibliothéconomique. Nous parlerons de l'arrimage conceptuel entre les jeux sérieux et la formation ludique en bibliothèque. En prenant l'exemple d'Escouade $B$, nous nous questionnerons sur le processus créatif et les phases de développement d'un jeu utilisé pour la formation aux

1. [En ligne] : < http://www.bibliomontreal.com/escouadeb >.

2. < http://ville.montreal.qc.ca/portal/page?_pageid=4276,5558134\&_dad=portal\&_schema=P0RTAL >. 
compétences informationnelles. L'objectif est de vérifier si un jeu sérieux comme Escouade $B$ peut répondre aux besoins d'un établissement pour aider à l'apprentissage, à la formation et à la diffusion de compétences informationnelles.

\section{LE JEU SÉRIEUX : ENTRE JEU VIDÉO ET JEU ÉDUCATIF}

Selon le théoricien Raph Koster, le plaisir dans le jeu provient de la capacité à comprendre un système structuré ${ }^{3}$. Un besoin intrinsèque nécessaire à notre évolution et à notre survie qui nous fait produire de la dopamine lors de notre compréhension ou de notre apprentissage d'un phénomène externe. Si le plaisir dans le jeu provenait de notre volonté d'apprendre, il n'y aurait qu'un pas à faire pour dire que le jeu pourrait être utilisé pour l'apprentissage de savoirs réels, tangibles et concrets à partir de concepts virtuels. C'est cette approche que tente de schématiser la théorie des jeux dits sérieux.

Escouade $B$ a été conçue selon le schème conceptuel des jeux sérieux. Soit, d'après la définition d'Alvarez, un logiciel combinant une intention " sérieuse » - de type pédagogique, informatif, marketing, idéologique ou d'entraînement - avec des ressorts ludiques. Le jeu sérieux de type edutainment (contraction d'education/entertainment) a comme premier objectif de transmettre du contenu et un savoir précis ${ }^{4}$.

\section{LE JEU SÉRIEUX : ÉVOLUTION}

Deux types de document ont influencé l'approche théorique du jeu sérieux : le jeu vidéo commercial et le jeu vidéo éducatif. Selon Vincent Berry, le jeu sérieux "part d'un jeu vidéo "fun" pour arriver à un jeu vidéo "éducatif" ${ }^{5}$. C'est-à-dire qu'il utilise les recherches sur le plaisir

3. Raph Koster, A Theory of Fun, Scottsdale, Paraglyph Press, 2004.

4. Julian Alvarez, Du jeu vidéo au serious game : approches culturelle, pragmatique et formelle, Thèse spécialité science de la communication et de l'information, sous la direction de JeanPierre Jessel et Gilles Methel : Toulouse, Université de Toulouse, 2007.

5. Vincent Berry, « Jouer pour apprendre : est-ce bien sérieux ? Réflexions théoriques sur les relations entre jeux et apprentissages ", Canadian Journal of Learning and Technology / Revue canadienne de l'apprentissage et de la technologie, 2011, vol. 2, n 37, p. 3. [En ligne] : < http://www. cjlt.ca/index.php/cjlt/article/view/606/313 >. 
effectuées par l'industrie des jeux vidéo commerciaux pour comprendre comment ils arrivent à transmettre leurs mécanismes aux joueurs. Les jeux sérieux remplacent les mécanismes intrinsèques du jeu par des notions éducatives.

\section{Les années 1980-1990}

Le jeu sérieux s'inspire donc de l'objectif de transmission du savoir présent dans le jeu éducatif, mais utilise des techniques modernes de design pour y arriver. Dans les années 1980 et 1990, le jeu vidéo éducatif a souvent eu recours à des thématiques de jeux vidéo pour " décorer » des jeux-questionnaires à choix de réponses multiples. Dans Mario Teaches Typing ${ }^{6}$, Mais où est Carmen Sandiego ? ${ }^{7}$ et Adibou ${ }^{8}$, le jeu propose une version augmentée d'un jeu-questionnaire avec un décor inspirant. Cette approche, encore utilisée de nos jours, est davantage inspirée des modules éducatifs et des examens scolaires que des mécaniques de jeux vidéo. Une autre approche du jeu éducatif fut d'utiliser les concepts transmis par un jeu vidéo commercial pour enseigner des notions précises. Des jeux comme Civilization et Sim City ont été utilisés dans plusieurs cursus académiques pour enseigner des concepts historiques ou géographiques. Selon l'historien Aaron Whelchel, l'utilisation de Civilization III en classe est non seulement valable, mais souvent préférable à l'enseignement traditionnel ${ }^{9}$. Le jeu sérieux s'inspire de cette deuxième approche pour créer du contenu original qui répondrait à des enseignements précis. Comme le mentionne Vincent Berry, « les chercheurs ou des concepteurs de jeu inversent la problématique et s'intéressent en conséquence à la façon dont un jeu vidéo non pensé pour l'éducation, mais pour le divertissement peut développer des situations d'apprentissage ${ }^{10}$. Les mécaniques des jeux sérieux sont donc, finalement, davantage inspirées des adaptations de jeux vidéo

6. Interplay, Mario Teaches Typing, Japon, Nintendo, 2011.

7. Broderbund Software, Where in the world is Carmen Sandiego?, États-Unis, Broderbund Software, 1985.

8. Roland Oskian, Adibou, France, Coktel Vision, 1992.

9. Aaron Whelchel, "Using Civilizaiton Simulation Video Games in the World History Classroom", World History Connected, 2007, Vol. 4, n 2. [En ligne] : < http://worldhistoryconnected.press. illinois.edu/4.2/whelchel.html >.

10. Vincent, Berry, ibid. 
(jeu de tir à la première personne, défense de la tour, stratégie $4 \mathrm{X}$, jeu de rôle, etc.) que des jeux-questionnaires à choix multiples.

\section{Les années 2000}

C'est dans cette veine que l'on voit arriver le mouvement des jeux sérieux dans les années 2000. Le mouvement des jeux sérieux est propulsé par l'armée américaine qui développe des jeux de simulation militaire comme America's Army ${ }^{11}$ pour aider au recrutement. Dans les années suivantes, les jeux à connotation politique comme Food Force ${ }^{12}$ et Ayiti: The Cost of Life ${ }^{13}$ font leur apparition. L'ambition de ces jeux est de sensibiliser, d'informer les citoyens sur des situations complexes en utilisant les mécaniques du jeu vidéo ${ }^{14}$.

Une critique récurrente envers le jeu sérieux est qu'il donne l'impression d'être le parent pauvre des jeux vidéo commerciaux. En se comparant à des jeux comme Destiny possédant un budget de plus de 500 millions de dollars, les jeux sérieux possèdent des graphismes et des éléments de jouabilité plus rudimentaires. Il est donc important de se rappeler que le jeu sérieux est principalement un format d'apprentissage pédagogique. Il doit se comparer à une approche magistrale ou textuelle pour tenter de répondre aux attentes des jeunes. Il est donc fortement recommandé d'inclure le jeu sérieux à l'intérieur d'une formation et non d'espérer que les apprenants joueront à des jeux sérieux au lieu des jeux vidéo commerciaux lors de leurs temps libres.

Le défi de la création du jeu sérieux est donc dans l'équilibre entre sa volonté éducative et ses inspirations vidéo ludiques. D’un côté, mettre un trop grand accent sur l'éducatif fera perdre l'intérêt et la motivation intrinsèque aux jeux vidéo. De l'autre, une approche trop ludique diluera le contenu pédagogique à transmettre et se comparera maladroitement aux jeux vidéo commerciaux.

11. United States Army, America's Army, États-Unis, United States Army, 2002.

12. Deepend et Playerthree, Food Force, États-Unis, United Nations World Food Programme, 2005.

13. Global Kids et GameLab, Ayiti: The Cost of Life, États-Unis, Microsoft, 2006.

14. Le site Games for Change propose une large sélection de jeux ayant pour objectif de "promouvoir une société plus juste et plus équitable ». < http://www.gamesforchange.org >. 


\section{DÉVELOPPER LES COMPÉTENCES INFORMATIONNELLES DES PUBLICS : LA BIBLIOTHĖQUE COMME ALTERNATIVE}

\section{LE POTENTIEL ÉDUCATIF DU JEU}

La bibliothèque publique a pour mission de « soutenir à la fois l'auto formation ainsi que l'enseignement conventionnel à tous les niveaux " ainsi que de « faciliter le développement des compétences de base pour utiliser l'information et l'informatique ${ }^{15}$. Elle doit donc offrir des formations en soutien au milieu scolaire et aider aux développements des compétences informationnelles et technologiques dans une approche axée sur la formation adaptée et l'apprentissage alternatif.

La difficulté étant de motiver le public lors de formations optionnelles. Selon le formateur et blogueur Bruno Devauchelle : « Que les jeunes soient capables de se passionner pour des activités ludiques, individuelles ou collaboratives, numériques, fait rêver tout enseignant confronté aux difficultés de motivation, d'intérêt ou de concentration ${ }^{16}$. Le jeu vidéo susciterait un grand support motivationnel pour l'apprentissage à partir des techniques d'essai et d'erreur, la prise de risque et la volonté de collaborer. Depuis leur gain en popularité, les jeux sérieux sont soumis à plusieurs critiques sur leur potentiel éducatif. Selon les recherches de James Paul Gee, l'enseignement par les jeux permet de transmettre les « compétences du $\mathrm{xxI}^{\mathrm{e}}$ siècle », soit l'innovation, la pensée critique et systémique, le travail en équipe et avec des outils numériques, afin d'en faire des producteurs plutôt que des consommateurs de savoirs ${ }^{17}$.

15. Selon le manifeste de l'Unesco sur la bibliothèque publique.

16. Cité à partir de "Jeux sérieux : apprendre en jouant... jusqu'à l'université » de l'Université de Sherbrooke. [En ligne] : < http://www.usherbrooke.ca/ssf/veille-old/dossiers-de-veille/jeuxserieux/ >. Bruno Devauchelle, "Les jeux sérieux permettent-ils vraiment d'apprendre ? ", Café pédagogique, 18 avril 2011. [En ligne] : < http://www.cafepedagogique.net/lexpresso/ Pages/2011/04/18_Jeuxserieux01.aspx >.

17. James P. Gee, What Video Games Have to Teach Us about Learning and Literacy, New York, Palgrave MacMillan, 2004. 


\section{LA FORMATION AUX USAGERS}

Autrement dit, les jeux font apprendre d'une façon distincte, complémentaire à l'enseignement traditionnel. C'est dans cet esprit que les jeux sérieux représentent à la fois un outil de médiation pertinent pour la formation en bibliothèque, mais également, une approche novatrice pour aider à motiver et à retenir l'information. En prenant en considération les supports informatiques déjà présents en bibliothèque, ce type d'outil peut s'intégrer facilement dans les formations aux usagers.

Pour donner accès à des jeux sérieux, il existe plusieurs répertoires de jeux sérieux en ligne comme Thot Cursus ${ }^{18}$ et Seriousgame.be ${ }^{19}$. Ils sont composés de centaines de jeux sérieux touchant à différents domaines dont les nouvelles technologies, la politique, l'environnement, etc. Les jeux sérieux peuvent être utilisés pendant la formation lors de séances encadrées ou pour conclure la formation afin d'assurer la mémorisation de l'information. Pour ce qui est des formations aux compétences informationnelles, il existe, en ce moment, peu de jeux sérieux dédiés spécifiquement au sujet. Un des rares exemples est le jeu A Google a Day qui aide à développer des stratégies de recherche pour son moteur de recherche. Le jeu connaît un succès intéressant, mais il s'agit néanmoins d'un outil développant des compétences pour un seul moteur de recherche.

Cet exemple illustre bien une problématique importante dans les jeux sérieux : la précision du sujet abordé. Il n'est pas facile de justifier un investissement élevé en ressources humaines et financières pour ne traiter que d'un sujet précis. Il devient souvent difficile d'ajouter un support ludique adéquat et complet pour les formations sur les compétences informationnelles en bibliothèque.

\section{LE PROJET ESCOUADE B}

Comme déjà mentionné, le projet Escouade $B$ fut lancé dans le cadre du programme Bibliothèque à la rescousse. Ce dernier est un programme des bibliothèques de Montréal qui existe depuis dix ans. Chaque année,

18. $<$ http://cursus.edu/ >

19. < http://www.seriousgame.be/ >. 
ce sont plus de mille participants des écoles primaires et des groupes d'alphabétisation qui viennent chercher des connaissances informationnelles dans les bibliothèques.

Une des problématiques de Bibliothèque à la rescousse était de montrer la recherche de l'information sur le Web à travers un module d'animation traditionnel. Contrairement à la recherche dans le dictionnaire, à la compréhension de la chaîne du livre ou à la lecture, une démonstration de l'information sur le Web est beaucoup plus porteuse via un support informatique. Toutefois, le nombre de jeunes dans les groupes, souvent entre 20 et 30 jeunes, empêchait que tous les participants puissent chercher en même temps en ligne. Il était donc plus facile de développer un jeu qui pouvait maintenir l'intérêt des jeunes et permettait de jouer en groupe.

\section{LES GRANDS PRINCIPES}

Le jeu Escouade B commença par la recherche d'une mécanique de jeu adéquate. Rapidement, nous avons décidé d'utiliser un jeu de type portemonstre-trésor où chaque confrontation deviendrait un moment propice à évaluer la connaissance du joueur. Les jeux de porte-monstre-trésor sont un type de jeu de rôle où le héros devient de plus en plus puissant en combattant des monstres qui sont de plus en plus dangereux. D'autres principes de jeux ont été envisagés, mais l'avantage des jeux porte-monstre-trésor est d'offrir une multitude de rencontres qui permettent à chaque fois de transmettre des compétences ou des connaissances de plus en plus avancées. Un autre type de jeu qui aurait pu être intéressant est le jeu de puzzle, où la recherche d'informations aurait été nécessaire pour réussir la quête d'un joueur. Mais un jeu de ce type offre souvent peu de rejouabilité*.

\section{LE DÉCOR ET LES RÈGLES DU JEU}

Pour la thématique, nous avons décidé d'utiliser le métro de la Ville de Montréal, un endroit sombre, propice à la vision labyrinthique des portesmonstre-trésor et qui réfère directement à l'imagination des habitants. Cette thématique permettait aussi de développer une histoire et une narration simple pour le jeu. Dans Escouade B, le jeune incarne un détective qui doit repérer et combattre les six monstres ayant pris le contrôle du métro. 
À l'aide des bibliothécaires de l'équipe, nous avons, par la suite, mis sur pied une série de concepts que les jeunes doivent comprendre pour analyser un site web : son auteur, la date de création et de mise à jour, le vocabulaire et l'orthographe, la sécurité, la navigation ainsi que l'intention et l'origine de l'information. À chaque concept nous avons relié un monstre que le joueur devra vaincre dans un mini-jeu d'arcade*.

De plus, pour s'assurer de bien simuler la mécanique des jeux portemonstre-trésor, nous avons fourni des objets à amasser pour le joueur ainsi que des niveaux d'expérience pouvant débloquer une lampe de poche, des nouveaux costumes et d'autres éléments de personnalisation. Ceci permet de proposer une progression aux joueurs et de concrétiser l'évolution dans le jeu.

La programmation et l'animation du jeu ont été faites avec le logiciel Adobe Flash et son langage de programmation AdobeScript 3.0. Malgré le succès récent des applications mobiles et du HMTL 5, les postes informatiques en bibliothèque supportent plus aisément les applications Flash. La complexité de la programmation et de l'animation, les plus grands défis du projet, furent gérés en interne. La musique et certains dessins ont été développés par contrat en externe.

C'est à ce moment que nous intégrons une équipe de bibliothécaires pour développer le contenu lors des rencontres avec des monstres. Chaque rencontre propose deux sites web originaux conçus pour chaque question. Pour assurer de la rejouabilité, nous avons développé un algorithme produisant des erreurs aléatoires dans un des deux sites web. L'algorithme inclut aussi un visuel aléatoire pour chacune des questions, ce qui nous assure que les joueurs y jouant une seconde fois ne peuvent repérer facilement la réponse.

\section{LA PHASE DE TEST}

Au cours du développement, qui dura deux ans, nous avons eu plusieurs rencontres avec des classes pour aider au développement. Nos rencontres démontrèrent que les jeunes étaient très intéressés par le jeu, mais que les apprentissages se dissipaient rapidement après la phase de jeu. À chaque itération du jeu, nous proposons de nouvelles façons pour s'assurer de la mémorisation de l'information : questions supplémentaires, 
questions finales et indices. Ces ajouts connurent un certain succès, mais c'est surtout le canevas d'information qui s'avérera le plus utile lors de la transmission de l'information.

Depuis le lancement du jeu en septembre 2013, les animateurs de Bibliothèques à la rescousse effectuent un suivi régulier de son utilisation. Le jeu est devenu un outil incontournable pour former aux compétences informationnelles autour de l'information sur le Web. Selon la critique du site web Thot Cursus : «Escouade B est un jeu sérieux réussi. Il use de bonnes mécaniques du jeu vidéo tout en distillant des messages clairs à propos des habitudes à prendre sur la Toile. L'habillage sonore est très agréable aussi. Une partie complète peut durer entre 45 minutes et une heure et les enfants peuvent rejouer par la suite pour augmenter leur score et figurer aux classements. Seul bémol : il n'y a pas beaucoup de pages web différentes par ligne de métro. La répétitivité est donc un peu au rendez-vous. Néanmoins, le titre se doit d'être joué par les plus petits qui découvrent Internet. » ${ }^{20}$

\section{un pari risqué et réussi}

Avec du recul, la création d'un jeu sérieux en bibliothèque, avec les limites financières, matérielles et humaines, s'avère être un pari risqué. Quoique les raisons d'être du projet soient pertinentes, il s'en est fallu de peu pour qu'à plusieurs moments, les objectifs ne soient pas atteints. De plus, sans budget pour un développement confié à des professionnels, le projet résidait en grande partie sur le dévouement et les compétences disponibles en interne.

Néanmoins, après deux ans, le résultat fut à la hauteur de nos attentes. Avec ce premier jeu sérieux, nous avons été capables de prouver l'intérêt et l'importance d'un tel projet. Les commentaires en ligne, plus de 500 en ce moment, prouvent l'intérêt des jeunes à interagir en ligne. Avec le soutien des animateurs de Bibliothèques à la rescousse, Escouade B connaît un grand succès et est devenu un outil essentiel dans le cadre d'une formation aux compétences informationnelles pour les 9-12 ans.

20. $<$ http://cursus.edu/institutions-formations-ressources/formation/21223/jeu-serieux-pour-apprendre-reperer-bonne/\#.VD1TctLuLE0 >. 
La popularité et la structure conceptuelle du jeu sérieux sont arrivées aujourd'hui à maturité, ce qui devrait inciter les bibliothécaires à les inclure dans leurs formations. Pour ce qui est de la création d'un jeu sérieux original, il s'agit encore d'un luxe que peu de bibliothèques peuvent financer. La recherche de partenariats avec l'industrie du jeu sérieux pourrait être une piste de financement pour développer des jeux utiles aux besoins spécifiques des bibliothèques. 
5

LES LUDO'MALLES DE LA MÉDIATHĖQUE DÉPARTEMENTALE DU PUY-DE-DÔME : UN OUTIL LÉGER POUR UN AUTRE REGARD SUR LES BIBLIOTHĖQUES

\section{À L'ORIGINE DES LUDO'MALLES}

Dans le cadre de l'étude réalisée en 2010 pour le second Schéma de la lecture publique, la question de l'ouverture d'un service "Ludothèque » se pose à la médiathèque départementale (MD 63) ${ }^{1}$, à l'image de la bibliothèque départementale de prêt (BDP) du Cantal ou de bibliothèques-ludothèques du territoire. Les ludothèques fleurissent un peu partout dans le paysage culturel à cette période, stimulant une demande croissante. Le projet est néanmoins vite abandonné en raison de sa lourdeur, au profit de la création d'outils plus légers intégrant le jeu, au même titre que les tapis d'animation, expositions, et autres supports d'animation prêtés aux bibliothèques du réseau, dont le succès et la facilité d'utilisation ne se démentent pas.

\section{POURQUDI DES MALLES DE « JEUX » ?}

Le « jeu » fait son entrée au catalogue sous le terme de "Ludo'malle », avec pour objectif de :

- créer un outil de dynamisation, participant de la mission des bibliothèques d'être des lieux de rencontres, créatrices de lien social, par le biais d'un nouveau type de supports d'animation accessible ;

- poursuivre le développement d'une action culturelle sans lien direct avec les collections, contribuant à une nouvelle 
perception des lieux dédiés à la lecture et, plus largement, à une offre culturelle ;

- attirer, de fait, un public nouveau autour d'activités ludiques, conviviales et favorisant la mixité des générations.

En mai 2011, afin d'évaluer la pertinence du projet, une enquête en direction des bibliothèques du réseau est lancée par la MD 63 .

\section{UN DIAGNOSTIC DE TERRITOIRE, VERS UNE RÉPONSE ADAPTÉE}

Le résultat de l'enquête envoyée aux bibliothèques empruntant déjà des supports d'animation à la MD 63 est indubitablement positif. Elles sont très favorables à cette ouverture au jeu. Du côté des publics potentiels, les réponses sont variées : du jeune public aux adolescents ou aux jeunes adultes, en passant par l'intergénérationnel et le familial, tous les publics sont représentés.

Une partie de l'enquête cherche aussi à comprendre dans quelle proportion les bibliothèques jugent plutôt pertinent de prêter des jeux à leurs publics ou d'animer des séances à partir de jeux. Et c'est bien le soutien à la mise en place d'animations et non l'emprunt, qui prime largement. Enfin, il est demandé aux bibliothèques de se prononcer sur le type de jeux envisagés : jeux de plateau, de coopération, de société, jeux de cassetête et de logique, jeux de plein air mais aussi jeux interactifs, jeux de rôle, livres jeux, jeux éducatifs, jeux de construction et jeux géants, jeux en ligne, en réseau ou via une Wii, etc. Il en résulte autant de réponses que de propositions ! La diversité actuelle et l'offre pléthorique en matière de jeux exigeront de faire des choix.

Malgré leur enthousiasme, des craintes sont aussi exprimées de la part des bibliothécaires. N'étant pas « animateurs », ils se posent des questions quant au temps et aux moyens nécessaires pour la mise en place de telles actions. L'accompagnement de la MD 63 est alors primordial.

Grâce à cette enquête, la MD 63 dispose de solides éléments pour expérimenter ce nouveau type de supports d'animation, en apportant une réponse adaptée aux craintes et aux attentes des partenaires dans la mise en œuvre de cette première Ludo'malle. Sa mise en circulation est prévue à l'automne de cette même année. 


\section{DES CHOIX INDISPENSABLES}

La Ludo'malle sera donc prêtée uniquement dans le but d'organiser des animations. Le public « intergénérationnel » se révèle être le plus indiqué pour la création d'une première Ludo'malle. Elle aura valeur de test, avec ce double objectif : être facile à mettre en place et toucher un large public. La durée du prêt est fixée à un mois. La sélection mélangera tous les types de jeux, en nombre suffisant ${ }^{2}$ pour occuper une demi-journée complète et satisfaire un public varié. Elle favorisera aussi la rencontre des joueurs et la mise en place d'animations par des " non spécialistes ». Côté contenu, il est envisagé, au départ, de privilégier des jeux « multi-joueurs » mais aussi surdimensionnés*, attirant facilement un public "papillon ». Des jeux d'ambiance*, très conviviaux et des jeux du monde*, sont aussi envisagés. Cet éventail tendra également à élargir la culture ludique des futurs joueurs. Une fois cette sélection déterminée, il reste à construire un cahier des charges détaillant chacun des choix et les étapes de réalisation. Une équipe-projet est créée à la MD 63, composée de deux agents et d'une stagiaire, étudiante en Master Conduite de projets culturels. Personne n'étant expert du domaine, l'équipe s'appuiera sur les « forces vives » existantes dans l'environnement local.

\section{COMMENT PROCÉDER ?}

Commence alors un travail de prospection et de recherche de conseil lors de "fêtes du jeu », auprès de ludothèques du Puy-de-Dôme, d'animateurs de maisons des jeux, de la BDP du Cantal mais aussi de bibliothèques-ludothèques du territoire. Ces rencontres se révèlent nécessaires pour comprendre les catégories de jeux existantes, leurs usages et leurs succès auprès de différents publics. Tous ces acteurs sont d'une aide précieuse pour réaliser une sélection cohérente, découvrir les fournisseurs, les ressources locales, résoudre des problèmes techniques (notamment de classification ou de vérification des jeux au moment du retour) et s'éviter bien des écueils. Après avoir rencontré l'ensemble de ces professionnels, fournisseurs et associations, l'équipe formalise l'écriture de premiers 
contenus. Ce travail de réflexion est lié, dès cette étape, à l'élaboration d'un guide d'animation qui accompagnera la Ludo'malle, en appui d'objectifs de médiation forts.

\section{DeS JeuX SOIGNEUSEMENT ChOISIS}

Ainsi, les choix des jeux sont réalisés en croisant les avis des professionnels, en rencontrant des joueurs, en recueillant leurs témoignages et leurs façons d'aborder le jeu. Dans une volonté de diversité (et non d'exhaustivité, il existe tellement de jeux !) la Ludo'malle s'adresse à un public large, permettant aux bibliothèques qui emprunteront ce nouveau type de support d'animation de tirer parti de cette diversité et des différents niveaux de jeux, appropriés aux projets d'animation mis en place. La sélection comporte ainsi 20 jeux (pour un budget de 1000 euros en 2011) : 3 jeux surdimensionnés, 6 jeux de société, 5 jeux d'ambiance, 1 jeu d'éveil sensoriel, 1 jeu coopératif, 4 jeux du monde et de construction.

\section{Un lien étroit avec les fournisseurs et les fabricants locaux}

À chaque fois que cela est possible, les fournisseurs locaux sont privilégiés, dans un souci de soutien et de fidélité aux professionnels ayant apporté leurs conseils et fait preuve d'une grande disponibilité. Les frais de port et les problèmes de livraison sont de ce fait facilités. De la même façon, un fabricant de jeux en bois local est sollicité pour réaliser le conditionnement de la Ludo'malle : une grande caisse en bois avec des poignées, facilement transportable.

\section{LE GUIDE D'ACCOMPAGNEMENT : OUTIL DE MÉDIATION ESSENTIEL}

Pas de médiation possible sans un guide d'accompagnement, en réponse aux missions d'accompagnement de la MD 63. Intitulé Ludo'malle : une sélection de jeux pour tous !, cet outil essentiel se compose d'une trentaine de pages. Il est déterminant pour l'exploitation de la Ludo'malle. 


\section{ENCADRÉ LUDO'MALLE : MODE D'EMPLOI}

Le fascicule comporte les rubriques suivantes :

- une note d'intention : resitue le jeu comme objet culturel à part entière et encourage une optique intergénérationnelle, le jeu faisant appel à un univers commun et familier. Il est souligné que cette Ludo'malle ne s'adresse pas aux spécialistes, qu'elle vise à favoriser la rencontre entre les joueurs et à permettre une perception de la bibliothèque comme un lieu de partage ;

- le contenu de la Ludo'malle : le présent guide, la liste des jeux, leur valeur d'assurance, les outils de communication fournis (affiches), les règles des jeux (plastifiées en grand format), les fiches de vérification des jeux, une fiche d'anomalie ainsi qu'une fiche d'évaluation à retourner à la MD 63 à l'issue de l'emprunt ;

- la description de chaque jeu proposé : de son conditionnement (mode de ran- gement) jusque sa mise en place matérielle au sein de la bibliothèque à l'aide de photos ;

- la préparation des animations : la rubrique insiste sur une nécessaire anticipation des actions. Avec ou sans budget, de multiples pistes d'animations sont proposées, à partir de montage de séances proposant telle ou telle combinaison de jeux. Une bibliothèque peut ainsi prévoir des « cycles » d'animations « jeux ", adaptés à des objectifs, des niveaux et des publics différents. En somme, les jeux ne sont pas tous à utiliser en même temps, il est même vivement conseillé de faire des choix ;

- des conseils et des ressources : renseignent sur les ludothèques locales, les associations et les animateurs de jeux, festivals et fêtes du jeu en Auvergne et hors Auvergne, les boutiques spécialisées, les sites web généralistes ou spécialisés, etc.

\section{ÉVOLUTION : ÉVALUER ET FORMER}

Lors de la mise en circulation de la Ludo'malle, en septembre 2011, la MD 63 organise une matinée festive de présentation auprès des bibliothèques en présence d'une animatrice de la maison des jeux locale. Parallèlement, le plan de formation de la MD 63 intègre dès fin 2011 des sessions régulières autour du thème " Jeu en bibliothèque ». Immédiatement, la Ludo'malle rencontre un grand succès. L'offre d'une seule Ludo'malle pose rapidement problème, ne permettant pas de répondre aux nombreuses demandes des bibliothèques. La réalisation de nouvelles $\mathrm{Lu}$ do'malles est décidée dans la foulée, tenant compte des retours et bilans 
récoltés au fil des premiers emprunts. Une fiche d'évaluation ${ }^{3}$ est ainsi créée par l'équipe pour savoir comment la Ludo'malle est exploitée, quels sont ses atouts et ses faiblesses. Ces évaluations sont un outil de suivi très efficace.

Au fil du temps, la Ludo'malle s'est révélée tout à fait adaptée aux besoins et a intégré naturellement les projets d'animations sur le territoire. Certaines structures ont même fait l'acquisition de jeux pour animer leurs propres séances. Sans se substituer aux ludothèques, cette nouvelle offre participe pleinement de ces objectifs de dynamisation et de désacralisation à la conquête de nouveaux publics. Tout en maintenant la bibliothèque à sa juste place : celle d'un équipement culturel de proximité.

\section{Et après ?}

Entre 2012 et 2013, la Ludo'malle 2 (jeux uniquement) et la Ludo'malle numérique (mélange de jeux sur tablette et de jeux physiques) voient le jour. Elles sont suivies en 2014 de deux Ludo'malles dédiées uniquement au jeune public (0-3 ans et 3-6 ans) et la collection devrait s'accroître, en 2016, avec trois nouveaux supports : une Ludo'malle 3 " tout public », une Ludo'malle « bidouille » (sensibilisation aux codes informatiques) et une Ludo'malle numérique pour les tout-petits (4-6 ans).

3. Exemples d'items : adéquation de la Ludo'malle avec les attentes ; adaptation aux besoins ; difficultés rencontrées ; motifs de satisfaction ; animations mises en place ; public ciblé ; partenaires ; communication ; réception chez les publics ; impact ; propositions d'amélioration, etc. 


\section{PARTIE III}

\section{ANIMER, CRÉER}

1. POURQUOI ALLER JOUER DANS UNE BIBLIOTHĖQUE ?

par Julien Devriendt

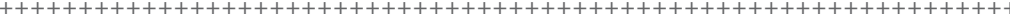

2. LE JEU COMME OUTIL DE CRÉATION : L'UNIVERS DE TOBIE LOLNESS SUR MINECRAFT. RETOUR D'EXPÉRIENCE SUR UN TRAVAIL MENÉ AVEC DES COLLÉGIENS

par Véronique Amar

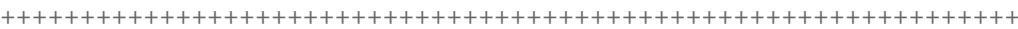

3. JOUER POUR JOUER

par Caroline Makosza

$++++++++++++++++++++++++++++++++++++++++++++++++++++++++++++++++$ 


\section{1 \\ POURQUOI ALLER JOUER DANS UNE BIBLIOTHĖQUE ? \\ par \\ Julien \\ Devriendt}

\section{LES CONDITIONS DU JEU}

Animateur socioculturel de formation avec une spécialisation technologies de l'information et de la communication (TIC), avant de travailler en médiathèque, j’ai travaillé en service jeunesse. Parmi mes collègues, des animateurs spécialisés en sport proposaient des activités autour du foot et remportaient toujours beaucoup de succès. Pourquoi ces jeunes allaientils jouer au foot avec l'animateur alors qu'ils pouvaient simplement jouer avec leurs amis?

Jouer au foot ne nécessite pas un équipement important : un ballon, des manteaux pour faire les buts et on peut jouer quasiment n'importe où. On joue avec ses amis, on ne compte pas vraiment les points, les règles peuvent être fluctuantes mais le plaisir du jeu est là.

Par ailleurs, chaque week-end, des milliers de jeunes se retrouvent sur de vrais terrains, avec des lignes blanches, de vrais buts, équipés de maillot, protège-tibias, crampons. Il y a également un arbitre pour qui les règles sont très claires, un entraîneur sur le bord du terrain donnant ses consignes et qui établit un plan de jeu et enfin un public qui prend des photos, encourage, supporte et commente les actions des joueurs.

Dans les deux cas, si on regarde l'activité de loin, l'objectif des participants est de pousser la balle dans le but du camp adverse. Pourtant, si vous participez à chacune de ces parties vous n'allez pas vivre la même expérience de jeu. D'un côté, on joue pour le plaisir de se retrouver entre amis sans autre objectif que de passer un bon moment. De l'autre, il s'agit non seulement de gagner le match mais aussi de réussir à se classer pour la phase finale du tournoi/championnat. 


\section{LE JEU COMME EXPÉRIENCE}

L'expérience de jeu, ce sont les conditions dans lesquelles le jeu va être joué, Pourquoi irais-je jouer à la bibliothèque aux jeux vidéo alors que je suis bien installé dans mon canapé, au calme, sans lecteurs ou bibliothécaires pour me déranger ? $^{1}$

Pour Scott Nicholson, auteur de Everyone Plays at the Library², l'important ce n'est pas le jeu, mais l'expérience de jeu. On va venir à la bibliothèque parce qu'on nous donne accès non seulement à des jeux mais à une expérience de jeu. Cette expérience de jeu peut être différente, à la fois par le jeu lui-même en proposant une sélection de jeux originaux et peu connus du grand public ou en proposant une manière différente de jouer à un jeu que le public possède déjà.

\section{L'heure du conte numérique}

L'heure du conte numérique proposée par Laurent Hautbout ${ }^{3}$ et reprise par d'autres bibliothèques dont la Petite bibliothèque ronde en est un parfait exemple. Pour mettre en place cette animation on utilise un jeu d'aventure de type point and click*, projeté sur un grand écran. Le public est en face, le médiateur contrôle la tablette (ou la souris) et demande l'aide des participants pour résoudre les énigmes et progresser dans l'histoire. Que vous jouiez à Monkey Island chez vous ou à la bibliothèque, le contenu du jeu utilisé est toujours identique. Cependant, le fait de jouer à plusieurs à un jeu pensé pour un seul joueur change radicalement l'expérience de jeu en l'enrichissant d'une dimension sociale jusque-là absente.

1. Lire Julien Devriendt : «Silence on joue ! Le médiateur, les jeux vidéo et les fonds documentaires », in Xavier Galaup (dir.), Développer la médiation documentaire numérique, Villeurbanne, Presses de l'enssib, 2012 (coll. La Boîte à outils ; 25). [En ligne] : < http://mediationdoc.enssib.fr/ lire-en-ligne/sommaire/iv-interagir-en-ligne-produire-des-contenus-partager/silence-on-jouele-mediateur-les-jeux-video-et-les-fondsdocumentaires >.

2. Scott Nicholson, Everyone Plays at the Library: Creating Great Gaming Experiences for All Ages, Medford, Information Today, 2010.

3. Laurent Hautbout, "L'heure du conte... interactif : (une utilisation possible du jeu vidéo en bibliothèque) », Jeux vidéo et bibliothèques, 2010. [En ligne] : <http://www.jvbib.com/blog/index. $\mathrm{php} /$ lheure-du-conte-interactif-une-utilisation-possible-du-jeu-video-en-bibliotheque/>. 


\section{Quand le jeu devient outil de création}

Certains jeux, via un éditeur de niveaux*, permettent de créer de nouveaux modes et manières de jouer. Defense of the Ancients (souvent abrégé en $\operatorname{DotA}$ ) fut créé à l'aide de l'éditeur de carte de Warcraft III avant de devenir un jeu à part entière. Chaque joueur peut créer son niveau et le faire tester par les autres joueurs. Le jeu peut devenir un outil de création, il est possible de se lancer dans un projet de création de jeux vidéo en utilisant des logiciels dédiés ou certains jeux « bac à sable », comme Minecraft, qui permettent toutes sortes d'exploration pour créer de nouveaux modes de jeux.

À Lyon, les élèves du collège Schoelcher en partenariat avec la bibliothèque municipale Anne Schwartz ${ }^{4}$ ont recréé l'univers de Tobi Lolness dans Minecraft ${ }^{5}$. Un projet très riche demandant aux élèves à la fois une connaissance approfondie de l'œuvre afin de créer les plans puis de passer à la réalisation, le tout de manière collaborative.

\section{L'EXPÉRIENCE DE JEU EN BIBLIOTHĖQUE}

Dans le chapitre 3 de son livre, Scott Nicholson propose un modèle de l'expérience de jeu en bibliothèque présentant les différentes interactions entre joueurs, spectateurs, personnels, et ressources.

\section{LES PROTAGONISTES}

Il y décrit le rôle de joueurs qui vont modifier l'état du jeu en bougeant les pièces du jeu ou en contrôlant un personnage à l'écran. Chaque joueur va ainsi modifier le jeu en suivant les règles fixées. Ces modifications peuvent les amener à interagir entre eux si le jeu l'exige ou simplement à discuter pour commenter la partie en cours ou même sans que le contenu de la conversation soit relié au jeu lui-même. Pour autant, cela fait toujours partie de l'expérience de jeu.

4. Carole Duguy, « L'univers de Tobie Lolness sur Minecraft par le collège Schoelcher », Blog Vive la culture numérique, 28 juin 2013. [En ligne] : < http://www.vive-laculturenumerique.org/index. php/2013/06/28/177-lunivers-de-tobie-lolness-sur-minecraft-par-le-college-schoelcher >.

5. Voir la contribution de Véronique Amar : « Le jeu comme outil de création : l'univers de Tobie Lolness sur Minecraft. Retour d'expérience sur un travail mené avec des collégiens », p. 102. 
Le cadre scénaristique du jeu est également à prendre en compte. Parfois anecdotique, " il faut sauver la princesse ", ou même inexistant, il peut se révéler extrêmement développé et au cœur même du système de jeu. Certains jeux se déroulent dans des univers préexistants dérivés d'autres médias (adaptation de romans ou de films) ou en prenant place à une période historique spécifique (seconde guerre mondiale, Renaissance, etc.).

\section{Les spectateurs}

L'auteur insiste ensuite sur l'importance du rôle des spectateurs. Ces derniers font également partie de l'expérience de jeu. Ainsi, on ne joue pas de la même manière tout seul chez soi, que lorsque 20 personnes vous regardent et commentent votre partie et vos actions.

Dans le documentaire Le tournoi des légendes ${ }^{6}$ tourné par Nesblog${ }^{7}$ et consacré au tournoi Street Fighter $2 x$ de l'Evolution Championship Series (EVO), un joueur expliquait la différence de niveau entre les joueurs japonais (qui dominent la discipline) et les joueurs européens et américains : selon lui, la différence tient à ce qu'il existe au Japon de nombreuses salles d'arcade*, permettant aux joueurs de s'entraîner avec cette pression du public, alors que les joueurs américains et européens, ayant moins régulièrement accès à des lieux où jouer en public, en dehors des tournois, ont plus de difficulté à supporter cette pression.

Bien souvent, les spectateurs sont composés de joueurs attendant leur tour, ils vont donc naturellement commenter la partie en cours, donner des conseils aux joueurs, discuter stratégie entre eux. La bibliothèque peut ainsi être un lieu de rassemblement pour des communautés de joueurs souhaitant se retrouver pour échanger autour d'une passion commune.

6. < http://www.jeuxvideo.com/chroniques-video/00000345/3615-usul-le-tournoi-des-legendes-4-4-00109747.htm >. Voir à partir de la $11^{\mathrm{e}}$ minute.

7. Le tournoi des légendes. [En ligne] : < http://www.nesblog.com/le-tournoi-des-legendes-episode-4/ >. 


\section{Les personnels}

Pour le personnel de la bibliothèque, plusieurs niveaux d'implication sont possibles : installer et lancer le jeu et rester en retrait (tout en s'assurant que tout se passe bien) ou avoir un rôle plus actif, en accompagnant les joueurs, voire même en complétant une équipe lors d'un tournoi multijoueurs. C'est une excellente occasion de faire connaissance avec le public. L'accompagnement peut aller au-delà de la simple explication des règles, en commentant une partie en cours par exemple. Cela permet à tout le monde de suivre, d'encourager les joueurs. Tout en montrant les éléments de stratégie du jeu, en explicitant les règles et la difficulté des actions réalisées aux non joueurs assistants à la rencontre.

\section{MONTER UN PROJET D'ANIMATION}

Enfin, les ressources de la médiathèque : c'est aux bibliothécaires de mettre en avant les ressources et les services proposés par l'équipement, de choisir les plus pertinents quant à l'animation proposée. L'univers de séries populaires comme Game of Throne ou The Walking Dead ont connu des déclinaisons en jeux vidéo, bandes dessinées et jeux de plateau. Il ne s'agit pas d'utiliser le jeu comme appât vers des pratiques jugées plus légitimes mais bien de le mettre au même niveau que le reste de l'offre de la bibliothèque et de proposer des passerelles entre documents, supports, afin de prolonger la découverte d'un univers.

Avant de pouvoir choisir le jeu, il est donc important de se questionner sur l'expérience de jeu que l'on souhaite proposer. Quelles relations entre les joueurs ? Entre les joueurs et les spectateurs ? Entre l'ensemble des participants et le personnel ? Entre les usagers et les participants ? Quelle relation avec les collections ? Etc.

Lorsque mes collègues animateurs proposaient une animation foot, ce n'était pas juste pour le plaisir de faire du foot, ils avaient des objectifs généraux, définis en fonction du projet de la structure qui les emploie et de leur public, qu'ils déclinaient en objectifs opérationnels. Cela peut aller du point bonus si au moins trois passes ont été faites avant de marquer, à la création d'une association portée par des jeunes pour monter un club de foot en salle, en passant par l'organisation d'un tournoi inter-quartier. Ce n'est pas très original, ni innovant, mais c'est la base du métier 
d'animateur. Cela permet de s'appuyer sur une pratique commune, motivante, pour responsabiliser, accepter les règles, respecter autrui, prendre confiance en soi, etc.

Il en va de même lorsque nous concevons un projet d'animation autour $\mathrm{du}$ jeu vidéo en bibliothèque. On part de la finalité, pour aller vers les objectifs généraux et enfin opérationnels qui nous permettront d'évaluer l'action menée.

CONCEVOIR UN PROJET D'ANIMATION AVEC LE JEU ENCADRÉ VIDÉO : 6 QUESTIONS CLÉS

- À quel public nous adressons-nous ? (la moyenne d'âge des joueurs est de 35 ans)

- De quel espace disposons-nous ? Est-ce que l'on peut faire du bruit? (est-ce que les joueurs ne vont pas être dérangés par le public ?)

- Sur combien de temps souhaitons-nous proposer cette animation ? S'agit-il d'une animation ponctuelle ou d'un club régulier?
- De quel équipement disposons-nous ? Disposons-nous d'un budget ? (Si vous démarrez, commencez avec PC et tablettes, il est toujours possible de brancher des manettes sur un PC et la majorité des titres y sont disponibles)

- Quels sont les autres espaces ou services proposés de la médiathèque que nous souhaitons mettre en avant?

- Avons-nous des personnes-ressources? Dans l'équipe ou parmi nos bénévoles/ vacataires?

Une fois formulées les réponses à ces questions nous pouvons choisir une expérience de jeu qui répondra à nos objectifs et sélectionner le jeu en conséquence. L'avantage de cette méthode est qu'elle oblige à prendre du recul sur son activité, et permet d'argumenter et de défendre auprès des élus, des équipes, collègues, usagers, la place du projet au sein de votre équipement. 


\section{2}

LE JEU COMME OUTIL DE

CRÉATION : L'UNIVERS DE TOBIE

par

Véronique

Amar

LOLNESS SUR MINECRAFT.

RETOUR D'EXPÉRIENCE SUR UN TRAVAIL

MENÉ AVEC DES COLLÉGIENS

\section{LE JEU COMME OUTIL DE CRÉATION}

D'avril à juin 2013, le réseau des bibliothèques de Lyon a proposé à son public « RéCréation », un événement se déployant sur 17 lieux et proposant 110 rendez-vous autour de la création contemporaine pour la jeunesse.

L'un des axes forts de RéCréation était "d'oser l'art numérique avant de prendre part à son tour à l'acte de création ». C'est dans cette ébullition créative qu'est né l'univers de Tobie Lolness sur le jeu Minecraft.

Ce projet s'est dessiné à partir d'une collaboration entre le secteur jeunesse et le secteur numérique de la bibliothèque Annie Schwartz, située dans le quartier de la Duchère et bénéficiant d'un contrat urbain de cohésion sociale (CUCS).

Une bibliothécaire jeunesse et une animatrice numérique se sont associées avec l'idée de travailler avec un auteur jeunesse, en imaginant un projet au-delà d'une rencontre avec des classes de collège, forme d'animation souvent proposée par les bibliothèques.

Une des idées fortes de ce projet était d'utiliser le jeu vidéo, d'une part, comme outil de création et, d'autre part, pour stimuler l'intérêt des élèves à la littérature.

\section{UN PARTENARIAT VERTUEUX}

La bibliothèque a alors proposé cette expérimentation au collège Victor Schoelcher par l'intermédiaire de la documentaliste. Celle-ci a organisé une rencontre avec deux professeures de français qui ont très rapidement montré leur intérêt pour ce projet, malgré l'aspect expérimental de cette proposition. Ces dernières ont proposé de mener cette expérience avec 
une classe de $4^{\mathrm{e}}$ de 26 élèves. Il a été planifié que l'œuvre choisie serait étudiée en classe d'octobre à janvier puis que les bibliothécaires co-animeraient avec les professeures des ateliers : 14 séances pour illustrer le roman sur un jeu vidéo. Les séances sur le jeu vidéo seraient menées avec la classe en demi-groupe tandis que l'autre demi-groupe participerait à un cours de soutien en français.

Il a également été prévu deux rencontres entre les élèves et l'auteur : la première au mois de janvier, après l'étude de l'œuvre et la deuxième au mois de juin. Cette deuxième rencontre, ouverte au public, serait l'occasion pour les élèves de présenter leur création à l'auteur et aux personnes présentes.

\section{POURQUOI TOBIE LOLNESS ?}

Le choix a été fait de travailler sur un roman présentant un univers fantastique afin de laisser toute la place possible à l'imaginaire des collégiens. Tobie Lolness est un personnage d'un millimètre et demi qui est poursuivi par son peuple dans un chêne.

$C^{\prime}$ 'est au cours des Assises internationales du roman ${ }^{1}$ à Lyon que les bibliothécaires ont eu la chance de rencontrer Timothée de Fombelle ${ }^{2}$ et de lui proposer cette expérience. Bien qu'il soit très sollicité, séduit par ce projet, l'auteur a accepté de voir son roman Tobie Lolness " adapté » sur Minecraft par une classe de collégiens.

Ce roman a été traduit en 28 langues. Il a reçu une vingtaine de prix français et internationaux parmi lesquels le prix anglais Marsh Award, le prix italien Andersen et la plupart des prix français consacrés à la littérature jeunesse. Au moment où ce projet a émergé, les droits d'adaptation au cinéma venaient d'être achetés par Amber Entertainement en vue de la sortie d'un film en 3D.

\section{POURQUOI MINECRAFT ?}

Il paraît important dans ce type d'animation de choisir l'outil en fonction de l'objectif et non l'inverse.

1. Les Assises internationales du roman : < http://www.villagillet.net/portail/air/actualites/ >.

2. Timothée de Fombelle, Tobie Lolness. Paris, Éditions Gallimard Jeunesse, 2010 (coll. Folio junior). 
Avec la parution d'outils de création de jeux vidéo comme Scratch ou Construct qui permettent de créer de façon simple des jeux de plateau, un premier choix s'est d'abord porté vers ce type de logiciels. Cependant, ces outils ne permettent pas d'avoir de graphismes sophistiqués et peuvent être compliqués à mettre en œuvre avec des groupes conséquents et une période limitée. Le résultat peut également se révéler décevant par rapport au temps investi dans la réalisation.

\section{Qu'est-ce que Minecraft ?}

Les vertus de Minecraft, en revanche, se sont montrées plus convaincantes. Créé en Suède, en 2010, au sein de la société Mojang par le suédois Markus " Notch » Persson, c'est l'un des plus grands succès de la scène des jeux vidéo indépendants de ces dernières années. En juillet 2014, il dépasse les 16 millions de ventes pour sa version PC et atteint les 54 millions d'exemplaires vendus toutes versions confondues. La société Microsoft vient de le racheter pour la modique somme de 2,5 milliards de dollars ${ }^{3}$.

Dans le même temps, il a fait son entrée dans les collections permanentes du Musée d'art moderne de New-York.

Classé dans la catégorie des jeux vidéo " bac à sable », le jeu génère en début de partie un environnement extrêmement vaste et propose deux modes d'utilisation. En mode "survie », le joueur devra réunir les ressources nécessaires pour subvenir à ses besoins et se défendre contre des monstres. En mode " création ", les ressources sont infinies et le joueur dispose de "blocs ", c'est-à-dire de cubes de toutes sortes qu'il dispose afin de construire ce qu'il souhaite, de la même façon qu'on pourrait utiliser des Lego®, mais virtuellement.

Le succès du jeu réside pour une grande part dans l'infinité des possibilités de création. C'est aussi l'une des raisons qui ont orienté le choix de Minecraft pour la conduite de ce projet. Une autre raison est la satisfaction immédiate qu'il procure dans la création de décors 3D.

En Suède ou aux États-Unis, il a d'ailleurs déjà été investi avec succès dans de nombreuses expériences pédagogiques ${ }^{4}$.

3. <http://fr.wikipedia.org/wiki/Minecraft>.

4. Alexandre Roberge, " Minecraft : du bricolage ludique aux usages pédagogiques », Thot Cursus, 
Il a également des qualités pratiques : c'est un jeu vidéo léger, qui ne nécessite pas d'avoir d'ordinateurs ou de système d'exploitation récents et s'installe facilement. Le prix d'achat du jeu n'est que de 20 euros.

\section{LE JEU RÉGULATEUR D'UN PROCESSUS DE CRÉATION COLLECTIVE}

Mais l'atout majeur de ce jeu est qu'il permet l'apprentissage de la difficulté à travailler ensemble.

S'il est possible de jouer seul, l'intérêt était ici de jouer en réseau. Les collégiens, placés dans le même espace virtuel, devaient construire ensemble au départ par groupe de six.

D'une manière générale, n'importe quel adulte se retrouvant dans un monde virtuel a comme premier réflexe de détruire ce que l'autre a construit ou de tuer les avatars en présence. Cela a d'ailleurs été la première réaction des enseignantes lors de leur séance d'appropriation du jeu avant les séances avec la classe. Pour des adolescents manquant parfois de cadre, cette réaction est démultipliée. Très vite à l'aise dans le jeu, ils ont notamment découvert spontanément les blocs de TNT et leur utilisation.

Ainsi, les premières séances ont souvent été de l'ordre du chaos virtuel. Le choix a été fait de laisser les deux groupes libres de leurs choix tout en les orientant avec des questionnements. À la première question «Est-ce que cela fonctionne dans votre groupe ? » les réponses étaient systématiquement du type «non, c'est l'horreur Madame, ils font n'importe quoi ». Suite à la suggestion de désigner un leader pour leur faciliter la tâche, souvent un ou plusieurs leaders se sont spontanément imposés au soulagement du reste du groupe qui pensait y trouver de la facilité. Les leaders se sont vite alors rendu compte que leur place n'est pas si facile à affirmer. Chacun se rend également compte au fur et à mesure des séances qu'il va devoir trouver sa place et une tâche à réaliser dans le groupe et

29 avril 2012. [En ligne] : < http://cursus.edu/article/18259/minecraft-bricolage-ludique-aux-usagespedagogiques/ > ; « Minecraft comme outil pédagogique et didactique », NetPublic, 19 janvier 2013. [En ligne] : < http://www.netpublic.fr/2013/01/minecraft-comme-outil-pedagogique/ >. 
dans l'arbre virtuel s'il souhaite que sa construction avance et se termine avec un résultat satisfaisant pour lui et pour les autres.

La construction collective permet également la compréhension de la nécessité de mettre en place des règles. Pour illustration, lors d'une des séances, alors que les arbres avaient pris forme suite au travail des élèves, l'un d'entre eux a mis volontairement le feu à l'un des arbres, ce qui a considérablement agacé ses camarades présents. En concertation avec les professeures, la décision a été prise non d'écarter de l'expérience l'élève « destructeur » mais de lui proposer des travaux virtuels d'intérêt collectif. Il lui a été demandé d'opter entre deux alternatives : reconstruire les décors endommagés au cours des premières séances par les «blocs » de TNT ou quitter le projet.

$\mathrm{Au}$ fur et à mesure des séances, deux arbres géants se sont élevés. Divisés ensuite par groupe de deux ou trois, nos joueurs ont créé les décors de Tobie Lolness : la maison d'Elisha, les villes de Joe Mitch, le lac, des fermes d'élevage d'insecte...

\section{LES DIFFICULTÉS RENCONTRÉES}

Il n'a pas été simple de mener une telle expérience et il a fallu faire face à plusieurs difficultés liées à sa mise en œuvre ou en cours de projet. Ce qui paraît aisé pour un particulier se révèle complexe à mettre en place dans une administration, particulièrement dans une structure aussi grande que celle de la Bibliothèque municipale de Lyon (BML).

\section{Acquérir et installer un jeu en réseau}

La première difficulté rencontrée a été celle de l'achat des « licences » du jeu. Le paiement en ligne sur le site officiel de Minecraft permet d'obtenir les codes d'un compte pour télécharger le jeu et ses mises à jour sur un ordinateur. Cet achat est possible uniquement en ligne, avec une carte de crédit dont ne disposait pas la bibliothèque à cette époque. La seule solution trouvée a été l'avance sur salaire d'un des agents de la bibliothèque, après accord avec la régisseuse.

Installer un jeu en réseau dans une structure publique est également un obstacle à franchir. Les bibliothèques de Lyon sont reliées par un réseau 
informatique dont plusieurs proxy assurent la sécurité. Il n'a pas été envisageable de faire lever ces sécurités pour animer les séances à l'espace numérique de la bibliothèque de la Duchère.

Heureusement, l'installation du réseau a été possible au collège. Les élèves qui souhaitaient continuer à travailler sur leur création à la bibliothèque ont pu le faire en nombre restreint car sur un nombre réduit de postes informatiques Minecraft est capable de détecter un réseau local et de s'y intégrer.

\section{L'adaptation}

Le temps et les contraintes liées à l'installation du jeu en réseau qui ont conduit à construire deux " arbres géants ", ont été mal évalués. Le roman de Timothée de Fombelle commence ainsi : « Tobie mesurait un millimètre et demi, ce qui n'était pas grand pour son âge $\aleph^{5}$. Même s'il n'a jamais été question de respecter les proportions du personnage du livre, construire des décors de cette taille s'est avéré chronophage. Lors du rendu final, lorsque les élèves ont présenté leur travail à l'auteur, celui-ci leur a expliqué que ce n'était facile pour aucun illustrateur. François Place, l'illustrateur de Tobie Lolness s'était lui-même questionné pour les proportions de ses dessins. Il a également été délicat de " faire du rond avec du carré ». L'univers de Tobie Lolness, qui vit dans un arbre au milieu de la nature, est tout en rondeur. Minecraft, quant à lui, propose des graphismes avec des éléments cubiques, donnant l'impression d'images très pixélisées et des animations rudimentaires, ce qui fait partie de son charme mais aussi de ses limites. Nos jeunes joueurs y ont mis toute leur adresse et leur créativité, ce qui leur a valu les compliments de l'auteur.

\section{Les moyens humains}

Enfin, au niveau des moyens humains, il a rapidement fallu prendre conscience que la présence de deux personnes était insuffisante pour mener à terme le projet. Heureusement, l'équipe de la bibliothèque de la Duchère s'est montrée solidaire. Les autres membres de l'équipe jeunesse sont venus en renfort, notamment pour la coanimation des séances au collège.

5. Timothée de Fombelle, Tobie Lolness. op. cit, p. 7. 


\section{LES ATOUTS DU PROJET ET SES APPORTS À COURT ET MOYEN TERME}

\section{POUR LES ÉLÈVES}

Cette expérience, qui s'est avérée pour les bibliothécaires, les professeures ainsi que la plupart des élèves plus que satisfaisante, a permis de comprendre que si le jeu vidéo est attractif, il permet aussi d'emmener facilement les adolescents vers des sujets qu'ils n'auraient pas forcément investis d'eux-mêmes.

Le jeu vidéo s'avère en effet un outil de médiation incontournable. Jouer avec des enfants ou des adolescents parfois peu loquaces ou peu enclins à ouvrir le dialogue avec des adultes permet d'ouvrir les portes à l'échange et à la complicité. C'est ce qu'explique par ailleurs clairement Serge Tisseron, docteur en psychologie et psychiatrie, dans son ouvrage Qui a peur des jeux vidéo ? $^{6}$

En outre, la dernière séance de présentation de l'univers créé par les élèves devant l'auteur en public a réellement placé les adolescents dans un rapport différent avec l'auteur, un rapport valorisant pour eux.

\section{Pour les bibliothécaires}

L'expérience acquise avec ce type d'animation a pu être réinvestie dans des animations à la bibliothèque. En effet, des ateliers sont régulièrement proposés aux plus petits dès l'âge de 7 ans. À titre d'exemple, ils peuvent aisément illustrer des éléments d'une histoire lue par une bibliothécaire sur Minecraft, comme cela a pu être fait, par exemple, avec la maison de la sorcière d'Hansel et Gretel en 2014.

D'autres applications sont également réalisables avec la création d'œuvre en Pixel Art 3D. Par exemple, l'animatrice numérique et la médiatrice culturelle de la bibliothèque Annie Schwartz ont pu faire participer les jeunes joueurs du club de football de l'Association sportive Lyon Duchère (ASLD) à une animation. En effet, l'ASLD amène les enfants et adolescents

6. Serge Tisseron, Isabelle Gravillon (collab.), Qui a peur des jeux vidéo ?, Paris, Albin Michel, 2008 (coll. Questions de parents). 
inscrits vers d'autres activités durant les vacances scolaires. Le thème retenu pour l'année 2013-2014 était l'art. Le Musée d'art contemporain de Lyon et la bibliothèque de la Duchère ont été associés à ce projet. Deux groupes de 11 adolescents ont été reçus à la bibliothèque. Au cours des quatre séances, Minecraft a permis d'initier ces adolescents à l'art numérique et de les faire participer à une œuvre collective. Les adolescents ont revisité à l'aide du jeu le célèbre tableau Guernica de Pablo Picasso ${ }^{7}$, ce qui s'est révélé une expérience très positive.

\section{UN « CLUB MINECRAFT » À LA BML}

Enfin, bien que les actions citées aient été menées avec un public «captif », le jeu vidéo peut aussi faire venir un public « non captif » à la bibliothèque. En effet, depuis le mois d'octobre 2014, il a été proposé aux usagers de participer à un « club Minecraft » un samedi matin toutes les trois semaines. Une dizaine d'enfants et adolescents viennent y jouer ensemble et réclament des séances supplémentaires. Lors des premières séances ils ont construit des bibliothèques !

L'équipe de la bibliothèque envisage plusieurs pistes afin d'utiliser cet outil de médiation pour emmener les enfants et adolescents vers la découverte et l'appropriation de leurs fonds.

Des projets comme la création d'une bande dessinée à partir de captures d'écran, en utilisant les décors construits sur le jeu ou un montage mêlant vidéo de Minecraft et films muets en noir et blanc, sont en cours de réflexion.

7. « Des footballeurs, des pixels, des cubes, de l'art.... », Blog Vive la culture numérique, 6 mai 2014. [En ligne] : < http://www.vive-laculturenumerique.org/index.php/2014/05/06/191-des-footballeurs-des-pixels-des-cubes-de-lart >. 


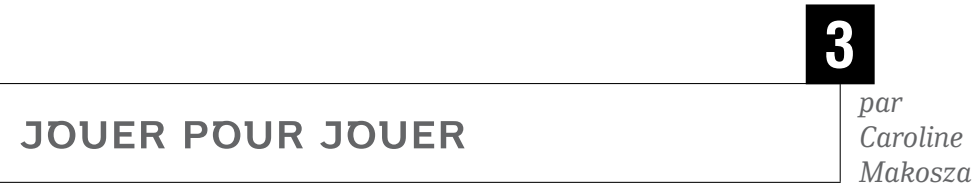

\section{UN PROJET EN MOUVEMENT : SIX ANS DE RÉFLEXION}

Les réflexions qui suivent sont issues du développement du projet de la ludo-médiathèque de Fosses (Val d'Oise, 9600 habitants, $500 \mathrm{~m}^{2}$ ) qui a ouvert ses portes en novembre 2012. Mais l'équipe proposait du jeu à la bibliothèque depuis 2008, dans ses petits locaux de moins de $100 \mathrm{~m}^{2}$. Nous avons donc eu beaucoup de temps pour construire notre réflexion sur la place du jeu dans les bibliothèques, sur le rôle des professionnels, sur l'image et l'impact de cette démarche aux yeux du public, des élus, des supérieurs hiérarchiques et des financeurs. Toute l'équipe a beaucoup réfléchi, s'est parfois disputé, a écrit, a lu, a convaincu, a inventé et a construit ce projet dont je vous livre ici quelques clés. Notre démarche s'appuie sur les formations, les livres, les articles, les recherches qui nous ont accompagnés et nous accompagnent encore.

Chaque projet est unique et s'inscrit dans un contexte, rien n'est transposable tel quel, ce n'est pas souhaitable et serait terriblement ennuyeux. Mais j'espère que les quelques éléments qui suivent pourront guider vos réflexions et vos pratiques dans vos projets autour du jeu.

Pendant tout le projet, et encore aujourd'hui, on a beaucoup ri et on s'est bien amusé. Si vous vous jetez dans cette aventure, je vous souhaite au moins autant de plaisir!

\section{DÉCIDER DE JOUER : LIBERTÉ POUR LES OURSONS !}

Le jeu en bibliothèque est encore bien souvent perçu comme un « produit d'appel » pour faire venir un hypothétique public et le guider petit à petit vers la lecture, activité légitime de la bibliothèque et souvent considérée comme seule digne d'intérêt.

Dans une optique tout aussi discutable, les bibliothèques vont chercher à donner au jeu une légitimation culturelle ou pédagogique en s'efforçant de 
mettre en lumière, de manière parfois un peu artificielle, ses côtés éducatifs (lecture, écriture, motricité, vivre-ensemble...). Les équipes vont alors construire des projets en se servant des objets (jouets, jeux de société, jeux vidéo...) comme d'outils d'animation, bien souvent en partenariat avec d'autres services ou institutions et sous le regard bienveillant d'élus et des différents responsables hiérarchiques. Je n'affirme pas ici que de telles actions soient toujours dénuées d'intérêt. Mais ce n'est pas du jeu. Appelons ça " loisirs dirigés ", " apprentissages ludiques ». Mais en aucun cas les participants ne vont jouer pendant cette activité. Ben oui, mais alors... Quand est-ce qu'on joue ? En quoi une activité est-elle un jeu ?

\section{QU'EST-CE QU'UNE SITUATION DE JEU ?}

Gilles Brougère ${ }^{1}$, dans la lignée de Roger Caillois, dégage plusieurs caractéristiques qui définissent une situation de jeu. L'une d'elles est la décision d'entrer ou de sortir du jeu. Le jeu lui-même peut se définir par une succession de décisions. Arrêter de décider, c'est arrêter de jouer. Seul le joueur peut choisir de jouer. Imposer à quelqu'un de jouer c'est risquer de détruire la situation de jeu. La seconde c'est la dimension de second degré de l'activité, le "pour de faux ». Quand on joue, on fait semblant. Par là même, ce que je fais dans le jeu, n’a pas de conséquence dans la vie réelle : c'est la " frivolité ». Ainsi, le loisir dirigé tel que souvent proposé dans les bibliothèques ne relève donc pas du "jouer ».

Certes, le jeu est plus facile à vendre quand on l'habille de tout un arsenal d'objectifs pédagogiques, parfois artificiels. Prenons garde à ne pas instrumentaliser le jeu d'une manière qui le débarrasserait largement d'un de ses plus grands intérêts pour en faire un outil d'animation pas tellement plus intéressant qu'une boîte de crayons d'une seule couleur². J'affirme d'ailleurs la même chose au sujet du livre : toute action qui vise à réduire le livre à un outil d'animation au service d'un projet vaguement

1. Gilles Brougère, Jouer - apprendre, Paris, Economica ; Anthropos, 2005 (coll. Éducation).

2. Voir à ce sujet l'intéressant article d'Eddy Léja-Six, "How Can Gameplay Allow Players to Get Creative?", Gamasutra.com, 2012, qui met notamment en lumière l'un des principaux intérêts créatifs du jeu Minecraft : permettre au joueur de fixer lui-même ses objectifs. Minecraft n'est plus un jeu si les objectifs assignés le sont par quelqu'un d'autre que par le joueur lui-même. [En ligne] : < http://www.gamasutra.com/view/feature/181915/how_can_gameplay_allow_ players_to_.php >. 
pédagogique (" faire aimer la lecture », « donner le goût de lire », « compléter les apprentissages scolaires ») est vide de sens et ne permet pas aux participants de votre activité de vivre leur propre expérience de lecture et d'en saisir l'intérêt, intérêt propre à chacun. Le livre, c'est pour lire ; le jeu, c'est pour jouer. Rien de plus, rien de moins.

\section{JOUER POUR JOUER : AUTANT EN EMPORTE LE FLOW}

Néanmoins, qu'il s'agisse de jouer ou de lire, comme il est difficile de résister à l'envie de fixer soi-même les objectifs de l'activité ! Car il s'agit là de résister à toute une conception de la société : celle de la productivité. "L'acte de jouer comme l'activité de la promenade sont aujourd'hui des actions liées à la pure perte lorsque la société contemporaine recherche la gestion, la rentabilité, l'utilité $»^{3}$. Dans le cas des animations telles que pratiquées par nos institutions (bibliothèques, centres de loisirs, accueils jeunesse...), on cherche bien souvent à ce que l'action entreprise et les agissements du public visé soient parfaitement maîtrisables par l'animateur. On vise alors une progression et des objectifs mesurables qui, dans l'idéal, devront être atteints par tout le groupe à la fin de l'action. Et surtout, il faut que l'activité " serve », qu'elle participe au développement des apprentissages que l'on jugera utiles aux enfants. L'activité doit conduire à sa réussite, surtout si elle est scolaire... mais pas forcément à son bonheur. Toute activité qui ne s'inscrit pas dans un schéma clairement productif et dirigé est alors jugée inutile ou, au mieux, activité de défouloir. Et pourtant... Certains chercheurs sautent le pas : « Les problèmes de la violence dans les banlieues des métropoles contemporaines proviennent, pour une bonne part, de ce que les innombrables activités éducatives, culturelles, sportives que la société propose aux jeunes sont de mauvais jeux et ne font pas jouer $»^{4}$.

3. Alain Guy, « Jouer, jouir du temps perdu ", Actes des universités d'été des ludothécaires, Paris, Association des ludothèques françaises, 1998. [En ligne] : < http://www.entre-vues.net/Archives/ Articles/tabid/635/ctl/ArticleView/mid/1242/articleId/27/JOUER-JOUIR-du-temps-perdu.aspx >.

4. Martine Mauriras-Bousquet, " Jouer et apprendre : même combat, le rôle des ludothèques dans la promotion du libre jeu », Ibid. 
Le jeu est une activité qui se suffit à elle-même, une activité autotélique qui, si elle n'est pas imposée mais librement choisie conduit à l'état de flow décrit par le psychologue Csíkszentmihályi ${ }^{5}$. C'est donc bien ça qu'on recherche : le jeu pour ce qu'il est, sans chercher à savoir quels apprentissages concrets l'enfant va en retirer. " Le jeu n'est éducatif que s'il ne cherche pas à l'être ${ }^{6}$. C'est sa propre expérience vécue à l'intérieur du jeu qui est intéressante pour peu qu'on l'ait choisie et qu'on la vive de manière libre et sans jugement.

\section{LE RÔLE DES BIBLIOTHÉCAIRES ET LA PLACE DES BIBLIOTHĖQUES}

Le rôle du professionnel se situe exactement ici : mettre à disposition un espace, des éléments, organiser des collections, des services et ensuite... laisser faire! Laissez les gens (et particulièrement les enfants) s'emparer de tout ce que nous mettons à leur disposition. Il faut qu'ils se sentent libres d'utiliser puis de s'approprier chaque objet (jouet, jeu, livre, jeu vidéo...) comme bon leur semble, sans jugement, sans objectif autre que le leur.

Le ludothécaire doit laisser jouer (et le bibliothécaire laisser lire). Il est là essentiellement pour organiser et réorganiser les espaces, accueillir, n'intervenir que si on lui demande, gérer les éventuels conflits, être présent, bienveillant et ranger... beaucoup beaucoup ranger. Ce non-interventionnisme est parfois difficile à accepter pour soi comme pour les autres... « Mais alors... vous ne faites rien ?! » s'entend-on parfois reprocher. Il y a beaucoup de choses à faire avant et après, mais pendant, non. Il ne faut surtout rien faire. Et ça n'est pas facile, pas facile du tout !

Avec ce positionnement professionnel, les conversations avec les parents et avec les enseignants seront à la fois riches et houleuses quand ils vous demanderont des jeux " pour apprendre à compter » comme lorsque, aujourd'hui, ils nous demandent des livres "pour apprendre à lire ». Or, on profite vraiment du jeu - et de tout ce qu'il peut apporter - quand on en maîtrise les mécanismes et les prérequis. Comme pour les livres... La littérature, c'est bien. C'est du plaisir. Mais ça peut l'être parce qu'on sait lire

5. Mihály Csíkszentmihályi, Vivre : la psychologie du bonheur, Paris, Robert Laffont, 2004 (coll. Réponses).

6. Martine Mauriras-Bousquet, op. cit. 
(ou parce qu'on vous lit à voix haute). L'acte d'apprendre à lire est difficile, même s'il peut être plaisant, comme tout apprentissage. Pour garder le plaisir de la lecture, on ne devrait apprendre à lire que dans de mauvais livres. Que ce soit livre ou jeu, si je ne maîtrise pas un tant soit peu les compétences nécessaires à la pratique, au mieux, je vais m'ennuyer, au pire, je vais associer cette expérience à une sensation désagréable. Or, nous avons tous besoin de réussite, surtout les enfants. Proposer à un enfant un jeu de Memory parce qu'il a une mauvaise mémoire, c'est le mettre en échec, là où il a besoin de réussite et de plaisir.

De nombreux chercheurs analysent comme marginale la place accordée au jeu libre dans la vie des individus et notamment des enfants. Il faut donc bien que quelqu'un se charge de (re-) donner au jeu sa place dans la vie des enfants et de faire en sorte qu'un temps non négligeable de jeu de qualité leur soit accordé. Alors les bibliothécaires, on s'y colle?

\section{UN PROJET DE SERVICE : LE SENS DE L'AVIS}

S’appuyer sur toutes les réflexions portées aujourd'hui par la recherche sur le jeu ${ }^{7}$ ne pourra qu'enrichir notre propre réflexion sur le rôle des bibliothèques. Penser le rôle du jeu, c'est penser le rôle du temps libre, des loisirs mais aussi celui de la littérature et de la culture dans la vie des gens. C'est aussi repenser (et il est vraiment temps !) les relations entre l'école et les bibliothèques, entre les apprentissages et les découvertes, entre le travail et le plaisir.

Introduire le « jouer » dans la bibliothèque, quels que soient les moyens dont on dispose, sera l'occasion de rédiger ou de re-questionner l'ensemble du projet d'établissement. Il ne s'agira pas simplement d'ajouter un nouveau service à son offre mais plutôt de repenser globalement toutes ses pratiques et postures professionnelles.

7. Voir notamment sur ce sujet le Centre de recherche interuniversitaire Expérience ressources culturelles éducation (EXPERICE) : < http://www.univ-paris13.fr/experice/ >. 


\section{LES COLLECTIONS : LOCALISATION, ESPACES, TRAITEMENT, USAGES}

\section{SÉPARER OU INTÉGRER ? : LE MEILLEUR DES DEUX MONDES}

L'idée qui vient le plus naturellement lorsqu'on pense à l'aménagement est d'imaginer des espaces " jeunesse », " adultes », " jeux », " CD et DVD » et « informatique » avec une organisation de l'équipe et des fonctions définies en conséquence. Or le monde n'est pas ainsi, considérons l'individu dans sa globalité, et c'est pourquoi de nombreuses bibliothèques sont aujourd'hui organisées différemment. Avec le jeu, c'est l'occasion de repenser ce modèle classique de l'aménagement et de l'organisation des équipes dans les bibliothèques. Du côté des professionnels, dans une équipe, nous avons tous le même métier. En pratique, tout le monde doit savoir à peu près tout faire. On vit tous la même chose. Dans l'esprit de notre projet, à Fosses, tout cela s'est construit de façon logique : il n'y a pas de concurrence entre d'éventuelles « sections » avec le risque redoutable de perdre la vision globale du projet et des gens et d'organiser sa réflexion, chacun de son côté, autour du support ou de la section que l'on s'est vu attribué. On parle souvent du « public au cœur de l'action ». C'est ce qu'on dit, c'est souvent ce qu'on ne fait pas. Qu'il soit jeu ou livre, en parlant plus de supports que de contenus, en organisant le métier et ses problématiques autour de ces éléments, on risque de s'éloigner de plus en plus des gens. Il ne doit pas y avoir de hiérarchie entre les supports. Ainsi, nous avons opté pour une prise en compte de la personne (faisant partie du public ou de l'équipe) comme une et indivisible et faisons de même avec notre projet. Il a une unité, un sens. Pourquoi le saucissonner ? Apprenons plutôt à relier les choses entre elles. Les espaces peuvent s'interpénétrer, les circulations peuvent être naturelles, fluides. Les supports et les pratiques peuvent communiquer, s'enrichir mutuellement. Et les gens aussi. 


\section{L'AMÉNAGEMENT INTÉRIEUR : MAISON ET SENTIMENTS}

On peut alors penser l'aménagement, non plus en terme de supports (livres jeunesse / adultes / jeux / etc.) mais en termes de pratiques et/ou d'ambiance. Ces espaces pourront s'enchaîner harmonieusement (peutêtre pas du premier coup...) et chacun pourra y trouver une place selon ses envies du moment. Chaque établissement devra tirer parti de ses propres recoins et autres bizarreries architecturales. Servez-vous-en, après vous en être plaint. Dessinez vos espaces avec ces éléments inamovibles ou bien avec des éléments de mobilier.

\section{FAVORISER LA VARIÉTÉ}

Pour les espaces de jeux de règles (les jeux de société en boîtes), pensez à proposer suffisamment de places assises et de tables de différentes tailles. Dans vos acquisitions, diversifiez les âges, les mécanismes, les éditeurs, les univers aussi.

Il me semble important que tous les supports de jeu soient présents dans une bibliothèque qui souhaite "donner à jouer » à son public, comme le fredonnent les ludothécaires... et nous ${ }^{8}$. À vous d'articuler au mieux cette nouvelle offre avec vos espaces existants ou d'autres que vous souhaitez proposer.

Bien des aménagements sont valables pour peu qu'on accepte de repenser les espaces traditionnels des bibliothèques. Si vous avez un ou plusieurs étages, vous pouvez vous en servir pour proposer d'autres ambiances. Il serait dommage de concentrer les jeux et jouets à un seul endroit alors que les pratiques liées aux différents types de jeux sont très disparates ! On ne joue pas aux échecs ou au Sudoku comme on joue à Halli Galli, ni à Counter Strike. Il n'y a pas que les adultes qui jouent au Scrabble, ni que les enfants qui jouent à Mario Party. Garder tous ces aspects à l'esprit permet de (re-)dessiner les aménagements. C'est un des moments les plus passionnants d'un nouveau projet et il est fortement lié aux objectifs fixés...

8. Voir à ce sujet l'encadré Les 5 espaces de jeux de la judo-médiathèque de Fosses, p. 67. 


\section{TRANSMETTRE UNE RÈGLE DU JEU}

Testez tout pour pouvoir expliquer. Testez également les différentes manières de transmettre une règle du jeu. Ça n'est pas toujours facile ! Chaque jeu est différent et il est difficile de donner une méthode d'explication de règles. De manière générale, commencez par planter le décor, s'il y en a un : les joueurs sont des explorateurs, des guerriers, des constructeurs, des poules, au Moyen Âge, dans le futur, au Far-West... Présentez ensuite le matériel de jeu : cartes, dés, plateaux, etc. Puis donnez le but du jeu : comment gagner. Ensuite, faites un ou deux tours « pour de faux » et expliquez les règles et mécanismes au fur et à mesure que les situations se présentent. Si vous le pouvez, jouez avec. Sinon, restez un peu pour dissiper les dernières interrogations, jusqu'à ce que la partie se déroule de manière fluide. Il arrive souvent que les enfants (ou même les adultes, d'ailleurs) inventent ou modifient les règles. Si on ne vous demande rien, n'intervenez pas. J'ai personnellement assisté à une partie d'échecs avec des pièces de petits chevaux et des cartes à jouer dont les règles furent pour moi tout à fait cryptiques mais dont les joueurs ont semblé retirer beaucoup de plaisir ! Lorsque tout sera en place : observez et écoutez. Prenez bien garde à la manière dont les gens vont s'approprier les lieux et analysez l'écart entre ce que vous pensiez et la réalité. Y a-t-il besoin de modifier quelque chose pour rendre les espaces de lecture plus confortable ou pour améliorer la qualité du jeu des enfants?

\section{ORGANISATION MATÉRIELLE ET FONCTIONNEMENT : CINQUANTE NUANCES DE PRÊTS}

« Et sinon? Vous recomptez toutes les pièces à chaque fois? ? Oui. On recompte à chaque fois. En réalité, on recompte à chaque retour et l'emprunteur, lui, est chargé de vérifier le contenu au moment où il emprunte. C'est loin d'être aussi pénible que ça en a l'air. Mais d'autres fonctionnements sont possibles. Certains utilisent même des balances de précision*... 


\section{La sélection}

En dehors de cet aspect, un jeu ou un jouet se traite de la même manière qu'un livre ou tout autre support. Vous allez fureter et faire vos sélections sur les sites web (Trictrac.net, jeudenim.be, jeusoc.fr, 1001jeux.free. fr, gamestories.fr), dans les salons (Playtime, Kidexpo, Festival du jeu de Cannes, Art to Play...) ou dans la presse (Plato) et les boutiques spécialisées. Évitez les grosses enseignes classiques si vous cherchez qualité et conseil. Il existe encore de nombreuses boutiques de jeux de société et de jouets de qualité en France, mais il faut chercher un peu, parfois faire quelques kilomètres. À défaut, tournez-vous vers la vente en ligne (réservoir-jeux, Philibert, avenue des jeux...) ou les enseignes professionnelles pour les gros achats et les investissements de base qui devront être de qualité (Haba, Wesco...).

\section{Le traitement documentaire}

Ensuite, vous pourrez cataloguer les objets acquis. Vous aurez peut-être besoin de faire évoluer votre logiciel. Selon vos objectifs, vous pourrez aller de la notice la plus simple à la plus complexe. Pareil pour l'indexation et la cotation. Il existe un système d'analyse de l'objet ludique qui est comparable à notre Dewey : le système $\mathrm{ESAR}^{9}$, un outil puissant qui permet une analyse très fine de chaque objet acquis. Cependant, et à l'instar de la Dewey, ce genre d'outil trouve ses limites lorsqu'on veut s'en servir comme système de classement et de cotation à destination du public. Le classement des objets ludiques (COL) porte les mêmes qualités et les mêmes défauts : un outil d'analyse, de conseil, de connaissance du fonds extrêmement complet et puissant mais moins efficace lorsqu'il s'agit de ranger et de coter de manière simple et intuitive. Nous avons, de notre côté, développé notre propre système de classement et de cotations basé sur les demandes des usagers.

9. Acronyme pour jeu d'exercice, jeu symbolique, jeu d'assemblage, jeu de règles. Voir Denise Garon, Le système ESAR : guide d'analyse, de classification et d'organisation d'une collection de jouets, Montréal, ASTED ; Paris, Éditions du Cercle de la Librairie, 2002. 


\section{L'équipement et les conditions d'utilisation}

Il vous reste à équiper tout ça... Commencez par consolider les boîtes qui vous sembleront fragiles, voire à changer complètement le contenant. Investissez dans des lots de boîtes cartons et plastiques vides, utile et pas cher. Bien que l'on retrouve souvent les règles de jeux sur Internet ${ }^{10}$, conservez-en tout de même une copie. Apposez éventuellement cote et code-barres sur la boîte avant de mettre en rayon.

Certaines ludothèques ne prêtent pas. Vous pouvez réserver les jeux à un usage sur place, tout dépend de votre projet. Fixez vos propres règles tout en veillant à ce qu'elles soient cohérentes avec le reste de vos supports. Dans tous les cas, à chaque étape, à chaque moment, réfléchissez et surtout... amusez-vous, amusez-vous, amusez-vous ! 


\section{PARTIE IV}

\section{PARTICIPER}

1. LA GAMIFICATION DE LA BIBLIOTHĖQUE par Thierry Robert

$++++++++++++++++++++++++++++++++++++++++++++++++++++++++++++++++$

2. COMMUNIQUER AUTREMENT AVEC LES ÉTUDIANTS : L'EXPÉRIENCE DU JEU EN RÉALITÉ ALTERNÉE À LA BIBLIOTHĖQUE MARIE CURIE DE L'INSA LYON

par Guillemette Trognot et Marie-Paule Voïta

$++++++++++++++++++++++++++++++++++++++++++++++++++++++++++++++++$

3. LA CHASSE AU TRÉSOR : DES LAPINS INTELLIGENTS À LA BIBLIOTHĖQUE

par Florence Carre

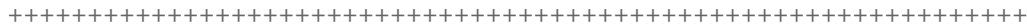

4. SE FORMER POUR ENTRER DANS LE JEU

par Julien Devriendt et Sophie Jacob

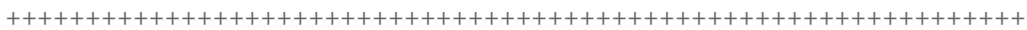




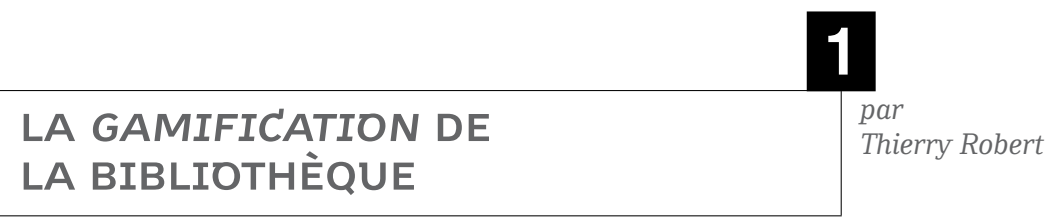

Depuis 2010, le terme « ludification » de l'anglais gamification ${ }^{1}$ gagne en popularité pour illustrer la multiplication des mécanismes ludiques, inspirées fortement du jeu vidéo, qui investissent différents domaines extérieurs au jeu. Les adeptes de la gamification croient que ces mécanismes augmenteraient l'appropriation, l'interactivité, la loyauté et le plaisir des usagers envers un service, une marque ou un produit. C'est principalement dans les secteurs des ressources humaines, de l'éducation et du marketing que l'on voit apparaître des approches de gamification à base de jeux sérieux, de badges, de points et de récompenses virtuelles pour motiver les usagers.

Les bibliothèques ne sont pas en reste dans cette nouvelle approche, nous allons jusqu'à postuler qu'elles sont même un espace public privilégié pour utiliser certains aspects de la ludification. Avec des techniques ludiques pour motiver à la lecture comme les récompenses et les échelles de pointage des clubs de lecture, plusieurs bibliothèques ont traditionnellement utilisé, souvent sans les nommer ainsi, des mécanismes de ludification pour donner goût à la lecture.

Les bibliothèques modernes, fortement inspirées des concepts du do-ityourself ${ }^{2}$, de l'apprentissage alternatif et de la bibliothèque troisième lieu, cherchent à offrir de l'information attractive en dehors de la logique scolaire. Différentes tentatives de ludification de l'espace, des services et des collections ont permis aux bibliothèques de se positionner comme des lieux ludiques, accessibles et motivants pour leurs usagers.

1. Le terme gamification a été utilisé pour la première fois par Nick Pelling. Par contre, le concept d'utiliser le jeu et le plaisir pour motiver les jeunes et les travailleurs se retrouve sous différentes formes à l'intérieur de la majorité des sociétés et des cultures.

2. L'arrivée en bibliothèque des imprimantes $3 \mathrm{D}$, des espaces de co-working et des Fablabs s'inscrit dans la mouvance du Do-It-Yourself, une approche visant à la création d'objets de façon artisanale. 


\section{GAMIFIER LE LIEU BIBLIOTHÈQUE}

Lors de ses recherches sur les joueurs, Richard Bartle a classé les motivations des joueurs à poursuivre leurs actions, selon quatre comportements : l'excellence, la compétition, la sociabilité et l'exploration ${ }^{3}$. Ce dernier - l'explorateur - représenterait la plus grande proportion des joueurs. Ce type de joueur veut surtout interagir avec le jeu et tenter de découvrir des éléments uniques hors des sentiers battus en testant les limites d'un environnement contrôlé.

\section{« EXPLORER » DANS LA BIBLIOTHĖQUE}

La mécanique du parcours ou de la chasse au trésor est un volet particulièrement populaire dans les techniques de ludification. Elle permet de modifier le comportement des usagers pour favoriser à découvrir ou redécouvrir un lieu donné dans une approche ludique et positive. La compagnie Google propose ainsi un jeu grandeur nature, Ingress ${ }^{4}$, qui incite les usagers à travailler en équipe pour mettre sur pied des portails virtuels à différents endroits à travers différentes villes. Avec ce jeu en ligne, Google recueille les données pour aider l'algorithme piétonnier de son service Google Maps. Misant sur la propension des joueurs à l'exploration, plusieurs bibliothèques de Montréal ont organisé la découverte de leur espace avec une approche ludique à faible teneur technologique. Chaque semaine, un objet, une mascotte ou un livre sont cachés dans un espace reculé de la bibliothèque. À l'entrée, un indice (gros plan, énigme, photographie, etc.) est donné aux usagers pour commencer le parcours et partir à la découverte des espaces cachés de la bibliothèque. Les usagers découvrent des espaces méconnus de leur bibliothèque en modifiant leur trajet habituel.

3. Richard A. Bartle, "Hearts, Clubs, Diamonds, Spades: Players Who Suit MUDs", 1996. [En ligne] : < http://www.mud.co.uk/richard/hcds.htm >.

4. Niantic Labs, Ingress, États-Unis, Google, 2012. 


\section{GAMIFIER LES COLLECTIONS EN BIBLIOTHĖQUE}

Dans son livre Reality is Broken ${ }^{5}$, la chercheuse Jane McGonical, conceptrice de jeux et figure dominante de la ludification, débattait de l'intérêt que pourrait avoir une application mobile qui servirait à motiver les lecteurs et à recenser leurs habitudes de lecture. Selon la conceptrice, la ludification est un procédé qui mobilise le meilleur en chacun de nous et devient un catalyseur positif pour atteindre des objectifs utiles et pertinents, comme la lutte contre la pauvreté, la défense de l'environnement et le développement de la lecture ${ }^{6}$. Jane McGonical tente d'utiliser la ludification pour répondre à des besoins réels à partir de la virtualité des jeux. Un exemple concret de cette utilisation positive est le jeu de puzzle Foldit ${ }^{7}$ où les réalisations des joueurs ont permis de résoudre un problème scientifique majeur sur le repliement des protéines. Le résultat du travail des joueurs a fait l'objet d'un article, publié dans la revue Nature ${ }^{8}$. Ce processus de motivation par le jeu a permis à transposer les compétences ludiques des joueurs vers un projet scientifique utile pour l'avancée de la science. Pour motiver à lire, les liseuses et applications de lecture sur tablette ont commencé à développer des fonctionnalités équivalentes. Les lecteurs sont encouragés à lire sous certaines contraintes pour acquérir des badges, des points et des récompenses virtuelles. L'application Kobo est particulièrement ludifiée avec plus de vingt badges qui s'activent lors de certaines pratiques de lecture ${ }^{9}$, un système de partage de lecture et des statistiques sur la lecture de l'usager.

5. Jane McGonical, Reality is Broken: Why Games Make us Better and How they Can Change the World, New York, Penguin Press, 2011.

6. Sa proposition Read+, est une application mobile encourageant les usagers à lire des ouvrages en les motivant à l'aide de badges et de récompenses virtuelles. Cette application est expliquée en détail dans Jane McGonical, Reality is Broken..., op. cit.

7. Seth Cooper, Foldit, Washington, University of Washington, 2008.

8. Firas Khatib, Frank Dimaio, Seth Cooper, Maciej Kazmierczyk, Miroslaw Gilski, Szymon Krzywda, Helena Zabranska, Iva Pichova, James Thompson, "Crystal structure of a monomeric retroviral protease solved by protein folding game players", Nature Structural \& Molecular Biology, 2011, Vol. 18, n 10, pp. 1175-1177.

9. [En ligne] : < http://www.kobobooks.com/awards >. Plusieurs badges sont donnés aux utilisateurs lors de lectures d'une certaine durée pendant la journée (la nuit, le matin, etc.) et en compilant diverses données (nombre de pages lues, nombre de livres lus, etc.). 
En bibliothèque, la ludification de la lecture commence par l'interaction entre l'usager et son dossier d'usager dans le catalogue. Les données amassées par les usagers (nombre d'emprunts, type de documents empruntés, frais de retard accumulé, etc.) peuvent servir à développer les bases d'applications ludifiées où les usagers pourraient voir les statistiques précises de leurs habitudes de lecture et les comparer à d'autres usagers. On voit déjà l'apparition de ces premiers catalogues ludifiés*10 dans certaines bibliothèques anglaises.

\section{GAMIFIER LES SERVICES ET LA PARTICIPATION}

La lecture n'est pas la seule approche que Jane McGonical propose de ludifier. La créatrice de jeu a développé Find the Future: The Game $e^{11}$ pour la New York Public Library (NYPL). Ce jeu en application mobile, disponible pour tous les usagers, a d'abord été proposé à plus de 500 adolescents ${ }^{12}$. Durant une nuit complète, hors des heures d'ouverture de la bibliothèque, ces adolescents, outillés de lecteurs de codes QR, ont envahi la NYPL pour y découvrir des artefacts et livres. À chaque étape, les joueurs devaient répondre à une question ouverte à laquelle l'équipe répondait ensemble. À la fin de la session, l'ensemble des réponses était imprimé dans un livre remis à chaque participant. Pour Jane McGonical, l'objectif du jeu Find the Future: The Game, était de permettre aux adolescents de découvrir leur propre futur à l'aide d'écrits et d'objets de personnages extraordinaires ${ }^{13}$. Cet exemple illustre comment une activité ludique, développée selon les meilleures pratiques de la ludification, peut favoriser le développement de compétences utiles à nos usagers.

Un autre exemple de la ludification des services est de motiver la participation des citoyens aux activités en bibliothèque. Dans le cadre du festival de jeux " Montréal joue ${ }^{14}$, les responsables ont décidé d'implanter un

\footnotetext{
10. Trois bibliothèques universitaires participent à Librarygame avec les catalogues ludifiés Orange Tree et Lemon Tree. Plus d'information : < http://librarygame.co.uk/ >.

11. < http://exhibitions.nypl.org/100/digital_fun/play_the_game >.

12. < http://www.nypl.org/blog/2011/04/01/jane-mcgonigal-and-nypl-present-find-future-game >.

13. < http://exhibitions.nypl.org/100/digital_fun/jane_mcgonigal >.

14. < http://www.montrealjoue.ca/ >.
} 
système de concurrence entre les différentes bibliothèques en temps réel sur le site web. L'objectif était d'augmenter la participation des usagers aux activités et de faire découvrir le site web. Ainsi, chaque usager participant donnait dix points à sa bibliothèque et le nombre de points était doublé si l'usager se connectait sur son compte en ligne. Pour motiver le personnel à faire participer les usagers, la bibliothèque ayant accumulé le plus de points était nommée " la bibliothèque la plus ludique du réseau » et avait la chance de gagner des prix dans un concours en ligne.

La ludification peut donc aussi être intégrée dans les services et programmes des bibliothèques pour attirer les publics et encourager les usagers à participer aux futures activités.

\section{LA GAMIFICATION : QUEL AVENIR EN BIBLIOTHĖQUE ?}

Au-delà de la ludification du lieu, de la lecture et des services, la ludification de la bibliothèque cherche aussi à changer son image. Elle permet de transformer la venue des usagers en bibliothèque en une expérience unique. Elle intègre le concept plus large de la bibliothèque troisième lieu où l'approche ludique est une des huit stratégies illustrées pour favoriser l'accès et l'appropriation du lieu ${ }^{15}$.

La littérature scientifique sur la ludification est encore en train de se rédiger et les bibliothèques sont souvent absentes des réflexions, et ce, malgré les différents prototypes que nous y développons. Si la ludification continue à progresser dans les prochaines années, il est important que les bibliothécaires s'intéressent rapidement à ce champ d'expertise où ils sont naturellement bien positionnés. Avec la structure conceptuelle apportée par le regain d'intérêt de la ludification, nous pourrons développer une approche ludique plus intéressante pour rallier différents types d'usagers. En plus de donner une image positive et ludique de la bibliothèque, cette technique pourrait aider à maintenir la fréquentation des usagers, leur

15. Les autres stratégies sont : être un terrain neutre, niveler les différences entre les gens, favoriser la conversation, être accessible et accommodant, créer des communautés, être facile et accueillant ainsi que devenir un second chez-soi. Voir Ray Oldenburg, The Great Good Place: Cafés, Coffee shops, Bookstores, Bars, Hair Salons and Other Hangouts at the Heart of the Community, Cambridge, Da Capo Press, 1989. 
faire vivre des expériences singulières, contribuer au développement de la lecture et favoriser la participation citoyenne.

C'est pour aider à la recherche sur le tiers lieu ludique que les responsables de la future bibliothèque de Pierrefonds ont mis sur pied une journée de co-design sur la ludification des bibliothèques. Des intervenants de l'industrie du jeu vidéo et du jeu de société se sont donné rendez-vous pour réfléchir aux meilleures pratiques pour intégrer des composantes ludiques dans une nouvelle construction de bibliothèque. Les conclusions de la journée furent que la ludification pouvait surtout avoir un impact majeur sur l'affichage en bibliothèque, la création de parcours augmentés, les activités hors-les-murs et les systèmes de recommandations ${ }^{16}$. Le futur de cette démarche permettra peut-être de jeter les bases de l'intégration réussie de la ludification dans les futures bibliothèques. 
COMMUNIQUER AUTREMENT AVEC LES ÉTUDIANTS : L'EXPÉRIENCE DU JEU EN RÉALITÉ ALTERNÉE À LA BIBLIOTHĖQUE MARIE CURIE DE L'INSA LYON

\section{2}

par

Guillemette

Trognot et

Marie-Paule

Voïta

La bibliothèque de l'INSA Lyon, école d'ingénieur, utilise des canaux classiques de communication : newsletters, mailing, portail documentaire, flux RSS, pages institutionnelles sur les réseaux sociaux.

Pourtant, cette communication ne génère que peu d'interactions avec le public étudiant qui reste passif et continue de voir la bibliothèque comme un lieu désuet tout en méconnaissant les services et ressources offerts. L'idée vient alors de demander à ce public de devenir acteur de la communication et pour cela de réaliser lui-même une action de communication qui saura le toucher : c'est ce qui a motivé la création, avec des étudiants, d'un jeu en réalité alternée. La rencontre entre les concepteurs du jeu et les bibliothécaires, leur vécu commun et les retombées sont présentées dans cette contribution.

\section{L'ORIGINE DU PROJET}

\section{LE CONTEXTE}

L'INSA Lyon accueille 6000 étudiants dont plus de $50 \%$ logent sur le campus. Ouverte en 2009, la bibliothèque Marie Curie (BMC) se situe en position centrale sur le campus ; elle propose environ $50 \%$ de ses collections en libre accès. Ce nouveau bâtiment a permis d'améliorer les conditions d'accueil, passant ainsi de 100 places assises à 650, avec des salles de travail en groupe, des carrels individuels, des salles de cours et même un amphithéâtre. De nouveaux équipements simplifient l'accès aux services et collections (automates de prêt et retour, accessibilité pour tous, wifi, etc.). De nombreuses animations multiplient les possibilités d'interactions avec les étudiants et les personnels. Les outils de communication 
sont modernisés : refonte du portail documentaire, flux RSS, newsletter, réseaux sociaux.

Le succès est au rendez-vous avec la multiplication par 4 de la fréquentation et notamment les salles de travail en groupe sont prises d'assaut, mais parallèlement, le nombre de prêt diminue.

\section{MÉCONNAISSANCE ET SOUS-UTILISATION DE LA BMC : CONSTATS}

\section{une représentation négative de la bibliothèque}

Différentes enquêtes d'opinion et mémoires d'étudiants de l'enssib ${ }^{1}$ montrent que les usagers (et non-usagers) ont une représentation des bibliothèques austère et élitiste : « la fréquentation de la bibliothèque est liée à l'idée que l'on s'en fait. ». Les étudiants doutent de l'utilité de la bibliothèque, un endroit vu comme obsolète depuis l'arrivée d’Internet. C'est ce que reprend également Pascal Siegel, dans sa présentation Les représentations des bibliothèques : «l'image de la bibliothèque est associée à la notion de travail, à la notion de lieu inaccessible (élitisme), de lieu un peu vieillot voué à évoluer $»^{2}$.

Par ailleurs, l'image du bibliothécaire reste austère : cet individu aime la lecture, aime que les livres soient bien rangés et fait respecter à outrance le règlement intérieur des bibliothèques.

À l'INSA Lyon, la bibliothèque n'est pas non plus forcément un lieu du quotidien comme l'est le restaurant universitaire ou les salles de cours. Une enquête auprès des étudiants de première et deuxième année montre qu'ils sont environ $40 \%$ à ne pas fréquenter la bibliothèque. Enfin, l'image de la bibliothèque reste assez élitiste même auprès des personnels administratifs, puisqu'une partie d'entre eux pense qu'elle est uniquement destinée aux étudiants et à la recherche.

1. < http://www.enssib.fr/les-poles-thematiques/histoire-des-bibliotheques/dossiers/la-representation-des-bibliotheques $>$.

2. Pascal Siegel, Les représentations des bibliothèques : l'impact des clichés culturels relatifs aux bibliothèques et aux bibliothécaires sur le public et les personnels, SCD Lille 3, 21 septembre 2011. [En ligne] : < http://fr.slideshare.net/hulot/les-reprsentations-des-bibliothques-limpact-desclichs-culturels-relatifs-aux-bibliothques-et-aux-bibliothcaires-sur-le-public-et-les-personnels$9425171>$. 


\section{L'inefficacité de la communication}

La politique de communication de la bibliothèque, au-delà de la transmission d'actualités, vise à faire connaître les services et les ressources. Mais, cette communication reste passive, poussée vers un public, sans demande expresse qui ne fait pas ou peu de retour. Peu d'étudiants commentent les actualités sauf sur le compte Facebook qui enregistre quelques réactions.

Craignant l'infobésité, les étudiants affirment ne plus pouvoir gérer leurs emails et faire un choix drastique sur les informations lues : ils suppriment les informations institutionnelles, non personnalisées qui sont perçues comme de l'information de masse qui ne leur est pas destinée directement. Ainsi, chaque envoi de la lettre d'information de la bibliothèque ne génère, en moyenne, que 300 clics.

Réfléchir à d'autres modes de communication devient essentiel.

\section{une méconnaissance des services et des collections}

Malgré les efforts de communication, malgré la présentation régulière de services personnalisés (poser une question, suggérer un achat, etc.), les services offerts demeurent peu utilisés voire méconnus. D’après les statistiques d'usages et les enquêtes réalisées auprès du public, seuls $10 \%$ des élèves connaissent l'existence des outils de recherche développés par la bibliothèque.

\section{NOUVEAUX OBJECTIF}

Fort de ce constat, plusieurs axes de travail sont explorés :

- aller là où se trouvent les étudiants : les réseaux sociaux, les associations (les étudiants de l'INSA Lyon font vivre près d'une centaine d'associations) ;

- utiliser leurs codes, comme sur la vidéo réalisée par des étudiants pour la BU d'Avignon ${ }^{3}$. 
L'idée émerge de travailler avec l'association vidéo, La Mouette ${ }^{4}$. Deux de leurs membres, Tanguy Pouzol et Brice Clocher, tous deux diplômés, sont approchés. Une vidéo, au ton amusant, leur est demandée ; l'idée leur plaît. Leur analyse du public-cible est la suivante : "Il s'agit de jeunes personnes dont les codes de communication sont typés. Ils sont sur-connectés et très réceptifs aux courtes formes médiatiques, au détriment de la communication classique telles que documents écrits, vidéos institutionnelles et didacticiels, auxquels ils n'accordent que peu d'attention. Leurs études et leurs activités sociales et associatives ne leur laissent pas le temps pour consulter du contenu dès lors qu'il ne leur paraît pas distrayant ou amusant. Ce projet doit toucher une corde sensible sur un plan personnel et émotionnel. »

\section{Créer un jeu vidéo}

Après plusieurs échanges et rencontres, deux propositions nous sont faites :

- une vidéo-web série : dix épisodes de quelques minutes diffusés sur les réseaux sociaux. Cette action se suffirait à elle-même, et ne demanderait pas d'implication de la part du personnel de la bibliothèque ;

- un jeu en réalité alternée (ou ARG pour Alternate Reality Game)* : il s'agit de raconter une histoire (une fiction) qui prend place dans le monde réel. Une série d'énigmes, avec la création d'une communauté de joueurs qui ne peuvent réussir qu'en s'associant en équipe. Ce jeu, transmédia, utilise de nombreux vecteurs (TV, vidéo, courrier, e-mail, téléphone, Web, article, forum, affiches...) pour développer un univers global et renforcer la crédibilité des situations.

Le principe du jeu en réalité alternée est retenu.

Nous parions sur plusieurs retombées : le jeu permettra d'attirer un public qui ne fréquente pas la bibliothèque, en l'incitant à réaliser une activité, de son plein gré, et à développer ainsi le plaisir du jeu associé au lieu bibliothèque. Outil de socialisation, le jeu favorise l'interactivité, la création de

4. < http://vie.asso.insa-lyon.fr/index.php/index-bot-insa/28-arts-et-spectacles/680-lamouette >. 
communautés et le partage des connaissances. Le jeu pousse les joueurs à réaliser des actions dont ils n'auraient jamais pris l'initiative comme utiliser certains services ou ressources tels que " Poser une question en ligne » qui deviennent ainsi connus. D'après Justin Kingsley (Sid Lee), et s'inspirant de Confucius : "Si tu me le dis, je vais l'oublier. Si tu me le montres, je vais comprendre. Si tu m'impliques, je vais m'en rappeler $»^{5}$, cette expérimentation a un fort pouvoir éducatif. Ainsi, le public, plus attiré et plus réceptif, s'impliquera plus naturellement, nous l'espérons, dans une relation plus intense, renouvelée et plus suivie.

\section{LE JEU EN RÉALITÉ ALTERNÉE}

Le jeu s'est déroulé en trois phases sur une durée d'environ deux mois.

\section{PHASE 1 - DAVID CONTRE GOLIATH EN MARS 2013}

Des événements marquants et vraisemblables mais fabriqués viennent perturber la vie du campus. Dans ce contexte d'incertitude, les étudiants apportent leur aide pour résoudre une situation sans précédent sur le campus, mais qui aideront-ils?

Dans un premier temps, une fausse société "Vemgråter ${ }^{6}$, lance une campagne de recrutement diffusée sur les réseaux sociaux de l'INSA Lyon et dans une campagne d'affichage. En une semaine, la société reçoit plus d'une vingtaine de CV.

Une semaine plus tard, un événement secoue le campus : un énorme tag apparaît sur le mur de la BMC ! Il est l'œuvre d'un certain Echométéore qui revendique le vol d'un document de Vemgråter stocké à la BMC. Il cherche à attirer l'attention des étudiants sur les zones d'ombre de Vemgråter, qui selon le document ferait courir un grave danger à l'ensemble du campus. Pour ce faire, il diffuse la vidéo du vol (sur Youtube) ${ }^{7}$, poste des articles sur son blog ${ }^{8}$, passe à la radio du campus (Gatsun) ${ }^{9}$. Les joueurs suivent la piste

5. < http://fr.slideshare.net/RobertGregoire_UdeM/dfinir-et-rfrencer-les-jeux-de-ralit-alterne >.

6. < www.vemgrater.com $>$.

7. < https://www.youtube.com/watch?v=qfz2gqazOeY >.

8. < https://echometeore.wordpress.com/ >.

9. < http://gatsun.asso.insa-lyon.fr/ >. 
que le voleur trace pour eux, découvrant tour à tour vidéo, articles et moyens de contacter le voleur (email, session de chat, etc.). Le voleur répond aux messages que les joueurs lui transmettent. De son côté, la société Vemgråter demande de l'aide aux étudiants pour identifier le voleur. Facebook, Twitter, chat sont utilisés pour interagir avec les joueurs. Lorsque suffisamment de gens écoutent le voleur, il révèle qu'il a disséminé les pages du mystérieux livre sur le campus. La phase prend fin lorsque Vemgråter finit par rattraper le voleur et l'enlève en direct lors d'une émission de radio.

Comme nous le rappellent les concepteurs : « Le jeu doit tout d'abord attirer l'attention du public, afin de recruter de nouveaux joueurs. Constamment entourés par les réseaux sociaux et les flux d'information, les étudiants ciblés n'en sont pas pour autant plus réceptifs aux nouveaux événements, surtout s'ils sont ambigus comme un ARG. De ce fait, nous comptons attirer l'attention du public en agissant dans sa réalité immédiate : la bibliothèque et le campus de l'INSA Lyon. En créant des événements intrigants, en affichant devant leurs yeux et en leur parlant directement, nous comptons atteindre les étudiants d'une manière plus percutante que les réseaux sociaux ne pourraient le faire, et ainsi favoriser les rumeurs et le bouche-à-oreille. »

\section{PHASE 2 - LA CHASSE AU « TRÉSOR », MI-MARS 2013}

Le jeu devient plus évident et visible avec la proposition d'une série d'énigmes ${ }^{10}$ à résoudre pour retrouver les pages du document volé et faire avancer l'histoire. Seuls ou en équipe, les joueurs résolvent les différentes énigmes qui les mènent sur tout le campus et, notamment, dans la bibliothèque.

Plus discrètement, les joueurs les plus hardis essaient parallèlement de retrouver la trace d'Echométéore. Dans cette tâche, ils auront l'aide inespérée d'une source au sein de Vemgråter. Piratage de compte email, documents secrets cachés dans les endroits les plus insolites de la bibliothèque et découverte tardive d'un ancien lieu de détention permettent d'élucider où est finalement retenu Echométéore et comment lui venir en aide. Une fois retrouvées toutes les pages du livre, cette phase prend fin. Les joueurs et leur complice se donnent rendez-vous à la bibliothèque pour anéantir la menace que représente Vemgråter. 


\section{PhASe 3 - Le déndUement, JeUdi 18 AVRIL 2013}

Le livre volé dévoile l'existence d'un artefact ancien et dangereux installé sur le campus. Les différentes pages permettent de comprendre sa fonction, son emplacement ainsi que son histoire, redécouvrant et réinventant au passage l'histoire du campus. Les joueurs découvrent finalement que l'artefact est au cœur du plot situé juste à côté de la bibliothèque ! (Le plot est l'œuvre financée par le $1 \%$ culturel de la bibliothèque). Ils se retrouvent donc lors d'une ultime soirée pour défaire cette influence néfaste sur le campus. Arrivés à la bibliothèque, ils y sont, malgré eux, scindés en deux groupes et enfermés. Malgré la séparation, les deux groupes collaborent pour trouver une issue commune et démanteler le plot. Le jeu se termine lors de cette soirée. Incités à collaborer et à réfléchir en équipe, à utiliser la bibliothèque dans toute sa spatialité (sous-sol, amphi, salles de TD...) et en dehors des heures d'ouverture au public, les étudiants font l'expérience d'une bibliothèque devenue le théâtre du jeu.

\section{ÉVALUATION DU JEU}

\section{Coûts}

- Coûts humains : plus de 1000 heures ont été nécessaires pour les deux concepteurs bénévoles. Les deux bibliothécaires ont consacré - outre des réunions hors temps de travail - deux semaines à temps complet soit 150 heures ;

- coûts financiers : la création de ce jeu a coûté 2740 euros répartis en $740 €$ pour le jeu (conception des sites web et du design des documents, divers accessoires) et $2000 €$ de nettoyage du mur de la BMC : en effet, le tag a été réalisé avec un produit à base de craie permettant un nettoyage aisé. C'était sans compter sur la mauvaise réaction du produit anti-tag existant. 


\section{Et les joueurs dans tout ça ? Et les non joueurs?}

Plusieurs éléments nous permettent d'analyser l'impact du jeu sur le public-cible des étudiants :

- statistiques sur les pages du jeu : la vidéo du vol sur Youtube a été vue 1600 fois, les sites web "Libérons le campus » et « Vemgråter » ont comptabilisé, chacun, plus de 10000 pages vues par plus de 1500 joueurs uniques ; quant au blog du voleur " Echométéore », ce sont près de 9000 pages qui ont été vues. Le buzz s'est créé également sur les réseaux sociaux : le premier post sur la page Facebook de l'INSA Lyon a généré près de 50 commentaires!;

- personnes sensibilisées : c'est difficile à quantifier. 51 joueurs se sont inscrits sur le site web "Libérons le campus » dont 27 ont joué réellement. Enfin lors de l'ultime soirée, il y a eu 15 participants. Nous n'avions pas d'objectif chiffré en nombre de joueurs. Le déploiement d'un jeu en réalité alternée se fait sans communication, le buzz des différents éléments du scénario devant suffire pour le faire connaître (principe du « trou de lapin »)*. Le ratio pages vues par des visiteurs uniques par rapport au nombre total d'étudiants est significatif de l'impact du jeu, même si le nombre final des joueurs est faible ;

- évaluation dans des focus groups : ils ont permis de rassembler des étudiants joueurs et non joueurs, ou non utilisateurs de la bibliothèque. Ces focus groups ont été l'occasion de faire s'exprimer le plus librement possible les étudiants sur leur perception du jeu, sur l'image qu'ils avaient de la bibliothèque, sur leur connaissance des services et de la communication. 19 non joueurs ont ainsi été auditionnés ainsi que 12 joueurs. 


\section{L'IMPACT DU JEU ET SES BÉNÉFICES}

\section{IMAGE}

L'image de la bibliothèque semble plus positive. La bibliothèque est perçue comme un lieu de tranquillité, de travail, de culture, de lecture, de détente, etc. L'image reliée au travail n'est pas vue comme une contrainte mais une nécessité. Enfin, ce jeu paraît être une bonne idée de communication, innovante.

\section{USAGES}

La moitié des étudiants ne vient que pendant les partiels ou pour la connexion Internet et la moitié n'emprunte pas ou peu de documents. Un tiers ne sait pas qu'il y a des romans et des bandes dessinées dans la bibliothèque. Globalement, les étudiants ne connaissent pas les services de la BMC alors que plusieurs ont été utilisés lors du jeu : "Poser une question », « Lecteur de microfiches », " Catalogue en ligne », " Documents de la réserve », mais il n'est pas certain que les étudiants vont réutiliser ces services pour leurs études.

\section{COMMUNICATION}

Les étudiants ont eu connaissance du jeu par Facebook, par le tag sur le mur, la vidéo sur Youtube, puis par le bouche-à-oreille, par des canaux alternatifs, donc. Plus globalement, la communication de masse utilisée habituellement par la bibliothèque est à conserver mais... elle est facilement « poubellisée » surtout si l'expéditeur est institutionnel et que l'objet de l'email n'est pas d'ordre personnel. Une communication personnalisée, permettant un contact individuel serait plus porteuse comme le flying par exemple. La communication communautaire serait à développer. Par exemple, chaque promotion a son groupe Facebook non officiel. Il ressort enfin la nécessité de créer des partenariats avec le Bureau des étudiants. 


\section{ÉVALUATION DE L'INFORMATION}

Suite au buzz lié au tag sur le mur, les étudiants ont eu deux types de réactions : ceux qui comprennent tout de suite qu'il s'agit d'un faux et ceux qui tombent dans le panneau, sont choqués puis comprennent par le bouche-à-oreille ou par le questionnement. De la même façon, lors de la campagne de recrutement de la société Vemgråter, une vingtaine de CV ont été reçus... alors qu'il suffisait de se rendre sur la page des crédits du site pour s'apercevoir qu'il s'agissait d'un jeu. Il a fallu se rendre à l'évidence, l'évaluation de l'information est loin d'être maîtrisée par tous.

\section{AU-DELÀ DU JEU}

Poursuivre la réflexion dans une table ronde, réunissant les concepteurs, les deux bibliothécaires, Patrick Perrier, sociologue et Patrick Prévot, enseignant expert en serious game de l'INSA Lyon et dans une série d'entretiens avec des chercheurs en communication (Eveline Manna, Céline N'Guyen) du centre des Humanités a atteint plusieurs objectifs : expliquer les raisons de ce choix d'un jeu, revenir sur cette expérience, sur les objectifs pédagogiques, analyser les questions que pose le jeu afin de réfléchir à l'impact fonctionnel sur les services de la bibliothèque et à la création de nouveaux projets :

- ce jeu visait la construction d'un comportement : venir et utiliser la bibliothèque. En dehors du jeu, on peut s’interroger sur ce qui amène le public à s'intéresser à la bibliothèque. Comment faire perdurer le lien créé entre la communauté des joueurs et la bibliothèque ? Une analyse poussée du comportement des non joueurs serait aussi intéressante ainsi que la création d'indicateurs qui ne porteraient pas forcément sur le jeu en luimême, mais qui seraient basés sur l'évolution des perceptions ;

- ce jeu laissait un flou entre réalité et fiction en mettant en question la crédulité des joueurs. Le jeu a montré que le public touché avait de fortes lacunes en termes d'évaluation de l'information et de sa fiabilité. Les concepteurs ont eu libre 
cours pour créer le scénario dans la microsociété que constitue l'école d'ingénieur. L'histoire du campus sert de trame (cf. les énigmes) au jeu avec plusieurs objectifs : valoriser le campus, garder le lien avec la réalité mais aussi donner une légitimité à ce jeu innovant mais perturbant. À travers le scénario, les deux concepteurs ont porté un regard critique sur les travers de certaines sociétés : par exemple, la manière dont Vemgråter réagit dans le jeu.

Ainsi ce jeu posait la question " peut-on tout se permettre pour communiquer autrement ? ». De nombreuses personnes ont été touchées voire perturbées par le tag sur le mur. (Rappelons que le tag était réalisé en matière nettoyable). L'idée que la dégradation appelle la dégradation est abordée. Cela interroge le fait que la microsociété de l'école a des difficultés à innover, à avoir des idées non convenues.

\section{Et ensuite ?}

Très clairement, ce projet a coûté cher et ne peut être pérennisé dans sa forme : il a été réalisé dans le cadre d'une opportunité de rencontre, d'envie d'innover/de créer, de temps disponible, d'objectifs clairs pour les deux parties : la BMC et les concepteurs.

Plusieurs points seraient à améliorer : le projet tel qu'il a été vécu n’a pas impliqué suffisamment le personnel de la bibliothèque qui n’a pas pu relayer le jeu. De même, si plusieurs structures associatives de l'INSA Lyon ont été sollicitées ainsi que la direction de la communication, il semble qu'il serait judicieux de compléter l'effet " trou de lapin » par l'implication de structures associatives fédératrices telles que le Bureau des étudiants. Enfin, les bibliothécaires d'autres établissements, voyant l'intérêt de ce type de projet, souhaiteraient être associés pour un projet de plus grande envergure.

Suite à cette expérience de jeu, de nouveaux projets sont en réflexion. Un jeu de géolocalisation a été développé par un groupe d'étudiants du département Génie industriel de l'INSA Lyon ${ }^{11}$ en 2013-2014, il a été lancé en 2014-2015. 
Du côté de nos concepteurs, Brice Clocher, ingénieur en Suède au début de l'aventure s'est reconverti en game designer*. Il travaille actuellement en Allemagne. Tanguy Pouzol, quant à lui, après une année sabbatique destiné à se former à l'écriture de scénario, est retourné pour l'instant à ses études initiales en démarrant une thèse.

Les deux bibliothécaires continuent de réfléchir à de nouveaux projets innovants. 
\begin{tabular}{l|l}
\multicolumn{2}{c}{$\mathbf{3}$} \\
LA CHASSE AU TRÉSOR : DES LAPINS & par \\
INTELLIGENTS À̀ LA BIBLIOTHĖQUe &
\end{tabular}

Automne 2003. Je viens d'entrer dans le métier et j'ai pris la direction de la médiathèque des Ulis. Une collègue qui travaille en section adulte vient me voir, visiblement très perturbée : "Florence, il y a deux messieurs qui sont installés, là, à une table, tu sais, celle qui est à côté des livres en langue

étrangère, et... (elle chuchote)... ils jouent aux petits chevaux... ils ne lisent pas. Qu'est-ce que je fais? ». Je la regarde, avec surprise : " Mais... quel est le prob...heu, ils ont l'air contents ? ». « Oui ». « Eh bien, c'est très bien, s'ils ont envie de jouer aux petits chevaux au milieu des livres, moi ça me va bien ». Elle repart perplexe.

Ville de 25000 habitants, cosmopolite, récente (créée en 1977), Les Ulis concentre une population socialement fragile (une partie du territoire est concernée par les " contrats de ville », avec quelques quartiers labellisés « zones urbaines sensibles » depuis longtemps) mais aussi de classes moyennes. La médiathèque des Ulis date de 1987, avec une architecture typique du style " cathédrale » de cette décennie. Elle a été transférée à la Communauté d'agglomération du Plateau de Saclay (CAPS) en 2013, et fait désormais partie d'un réseau de quinze médiathèques. La mise en réseau a accentué la mixité sociale : près d'un tiers de ses usagers vient des communes alentour à la CAPS.

Les directions successives ont toujours œuvré pour suivre de près les usages de la population et ont fait progressivement évoluer les services proposés, mettant au cœur du fonctionnement de l'équipement les outils et usages liés au multimédia, d'où, en l'an 2000, l'obtention du label Espace culture multimédia (ECM) attribué par la Direction générale des affaires culturelles (DRAC). 


\section{LA BIBLIOTHÈQUE HORS LES MURS}

L'équipe a l'habitude de monter des actions en direction du public éloigné du livre et de la lecture, en choisissant délibérément d'être présente sur le territoire, par exemple dans les services municipaux, dans les parcs, lors de forums ou de fêtes, ou encore au centre commercial Ulis 2 qui est le point névralgique de la cité, en apportant parfois sur son stand autre chose que des livres ${ }^{1}$, par exemple : des outils numériques (imprimante 3D) et des instruments de musique mis à disposition des usagers, pour casser son image de culture savante et se faire connaître auprès de nouveaux publics. Si, en 2000, les ordinateurs étaient soigneusement tenus à l'écart des collections physiques, et n'étaient présents que dans l'ECM, lui-même ouvert à des horaires distincts de ceux de la médiathèque, progressivement, des postes informatiques sont venus se fondre dans les espaces de l'équipement, signe que les ressources documentaires symboliques du cœur de métier et les outils multimédias ne s'opposaient nullement mais se complétaient, ce que démontrera une fois de plus le jeu dont il sera question dans cette contribution.

\section{FESTIVAL DES ARTS MÊLÉS 2012 : FABRIQUES NUMÉRIQUES}

En 2012, la directrice des affaires culturelles de la ville propose d'organiser le festival annuel de la médiathèque autour de la thématique du numérique et donne carte blanche à l'équipe pour penser l'événement. Le responsable de l'espace public numérique réfléchit alors à l'axe autour duquel envisager cet événement. Trop souvent ce qui a trait au numérique est limité à l'ordinateur et considéré comme virtuel et solitaire.

Les nouveaux outils modifiant en profondeur notre rapport à la culture, à l'accès à l'information, via les blogs, les tweets et les réseaux sociaux, il envisage de partir de l'existant pour montrer les tendances qui se dessinent et leurs impacts dans notre vie quotidienne. À travers cette découverte, les usagers pourront se rendre compte des réelles possibilités des outils et des enjeux qui existent aujourd'hui. Il s'agit de permettre aux

1. Je rassure les puristes, nous avons toujours apporter quelques livres, on aime juste provoquer et montrer autre chose que les évidences... 
usagers de se sentir citoyens dans un monde où le numérique devient de plus en plus présent.

Dans la liste des actions possibles, c'est le Do-It-Yourself, ou, dans la langue de Molière, "Fais-le toi-même » qui ressort. L'idée n'est aucunement de considérer les outils du numérique comme une fin en soi, mais de montrer leurs impacts dans le monde physique en permettant au public de manipuler du matériel lors d'ateliers pratiques de découverte et d'expérimentation.

Trois objectifs généraux sont alors déclinés :

- utiliser : acquérir des connaissances techniques ;

- comprendre : prendre en main, porter un regard critique, évaluer ;

- créer : créer et communiquer via des médias utilisant l'image, le son et la vidéo ; utiliser le contenu du Web 2.0 tels les blogs et les forums de discussion, le partage de photos et vidéos, les jeux sociaux et autres formes de médias sociaux.

L'action relatée ici se nomme " La chasse au trésor », et a été inspirée d'un jeu urbain réalisé par le Cube d'Issy les Moulineaux en $2010^{2}$.

\section{LA CHASSE AU TRÉSOR}

L'objectif affiché était de souligner la cohérence entre un jeu numérique et les missions « traditionnelles » des médiathèques, qui sont chargées de " contribuer à la culture, à l'information, à la formation et aux loisirs de tous les citoyens en mettant à disposition des ressources documentaires encyclopédiques et pluralistes. » (cf. le règlement intérieur des médiathèques de la CAPS).

Il semblait important de concevoir une action capable de tisser un lien fort entre la lecture publique et le virtuel, le numérique. Qui dit lecture publique, dit livre, donc écrivain et lecture, donc lecteur. Nous nous sommes mis en quête d'un écrivain pour écrire une histoire de chasse au trésor, et de lecteurs aguerris pour raconter cette chasse au trésor aux enfants.

2. < http://www.cubefestival.com/edition2010/jeu-urbain/ >. 
Le Groupe 3.5.81, en résidence à l'Espace culturel Boris Vian des Ulis, a proposé l'auteur Alain Bellet pour nous accompagner dans cette aventure, ainsi que plusieurs des comédiens du Groupe pour jouer l'histoire. Cette action était pour nous, de tout le festival numérique, la plus attractive puisqu'elle prenait pour socle ce qui fait une médiathèque : un auteur, une histoire, avec des usagers qui vont être amenés à vivre une expérience collective, et vivront en direct un jeu se déroulant dans les murs bien physiques d'une médiathèque. Le concept était alléchant.

\section{LES MOYENS MIS EN GEUVRE}

Nous décidons que le public-cible aura de 5 à 10 ans. Les outils numériques choisis sont des lapins appelés « Karotz » ou « lapin intelligent », de la société de robotique Aldebaran. Ces mini-robots sont connectés par wifi à Internet. Ils sont équipés d'une caméra, d'un système de reconnaissance vocale, d'un haut-parleur et d'un lecteur RFID.

Ils seront cachés à différents endroits de la médiathèque, et donneront des indices aidant à résoudre l'énigme principale pour arriver jusqu'au trésor. Les comédiens joueront l'histoire et guideront les enfants dans toute la médiathèque (soit $1800 \mathrm{~m}^{2}$ à parcourir). Les enfants devront présenter aux lapins des indices sous forme de carottes équipées de puces RFID. Ces puces RFID seront reconnues par le lapin qui délivrera ensuite ou non l'indice. Pour l'anecdote, n'ayant pas de wifi à la médiathèque, nous avons compensé en enregistrant des phrases audio dans des fichiers mp4 enregistrés dans des clés USB, qui fonctionnent aussi sur les lapins. L'ensemble de ce projet pilote a coûté aux alentours de 4000 euros, ce qui a permis de rémunérer l'auteur et la prestation des comédiens, les lapins ayant été mis à disposition gracieusement par Aldebaran.

Le matériel utilisé a été le suivant : huit lapins Karotz, 24 puces RFID, et des points électriques. En ce qui concerne l'accompagnement, trois comédiens du Groupe 3.5.81 et un agent de la médiathèque devaient être présents à chacune des séances. Il s'agissait de guider le groupe, permettre une médiation sur l'utilisation des lapins et de pallier les problèmes techniques éventuels tout en restant dans l'univers du jeu. Le personnel de l'espace jeunesse de la médiathèque a donc participé au projet, avec les deux agents de l'espace numérique. 


\section{L'ORGANISATION DU JEU}

Le programme du festival annonçait la chasse au trésor ainsi : « Jolicielle, Doubleclic et Arobaz ont quitté leur monde virtuel et douillet pour vous accompagner dans une chasse au trésor. Écoutez bien les lapins robots cachés dans la médiathèque et essayez de répondre à leurs questions pour tenter de retrouver le plus fantastique des trésors. »

Dix séances ont été organisées, cinq un mercredi, cinq un samedi, avec un départ toutes les heures et quart pour une chasse au trésor d'une heure. Les comédiens surgissent de la " carriole du livre », qui est utilisée l'été pour les lectures dans les parcs de la ville, et emmènent les enfants dans la chasse au trésor. Ils les conduisent au sein d'espaces dédiés au public, mais aussi sur la terrasse, dans la " tour infernale » (tour de l'escalier de sortie de secours) - nous aimons beaucoup jouer dans cette équipe et avons donné des noms étranges à nos lieux quotidiens : tour infernale, aquarium, salle du coffre. Les comédiens marquent un arrêt dans la réserve, et les enfants doivent s'employer, pour leur plus grande joie, à tourner les volants des compactus pour y découvrir, bien planqué sur un rayonnage entre des albums pour les bibliothèques-centres de documentation (BCD), le lapin "Internette », qui attend sagement qu'on lui passe une carotte sous le nez pour délivrer son message. Les enfants disposent chacun d'une feuille de route, qui leur indique les consignes suivantes :

\section{ENCADRÉ FEUILLE DE ROUTE DES ENFANTS}

« Notre jeu est personnel et collectif. Les bonnes réponses aux questions rapportent des points à chaque joueur, et parfois un joker au groupe, si l'un ou plusieurs d'entre vous trouve(nt) la bonne réponse. Attention, des robots vous attendent pour vous poser une ou plusieurs questions qui rapportent des points ».
Les questions comportent différents niveaux de difficulté, permettant aux enfants d'âge varié de jouer en équipe et de s'entraider : elles portent sur le nom des lapins, des mots à trouver.

Exemple : 
Trouve quatre mots importants :

un mot sentimental :

un mot joyeux :

un mot plein de douceur :

un mot plein de tristesse :

Si tu trouves les 4 mots tu gagnes 10 points

Si tu n'as pas tous les mots, tu peux jouer un joker que ton groupe a gagné.
Dans la liste que tu viens d'entendre, trouve :

un mot à apprivoiser :

un mot à éviter :

un mot à collectionner :

note ici le nom du robot :

Si les mots sont justes : 10 points

Le guide donne un joker à ton groupe : Arobaz donne une règle, Double-Clic un nez rouge, Jolicielle une craie de couleur.

L'imaginaire, l'écoute sont donc sollicités pour ce jeu.

\section{Bilan de l'action : un succès public}

Les dix représentations font le plein : 120 enfants participent, une vingtaine ne peut s'inscrire faute de place. Les enfants comme les parents sont ravis de la qualité de l'animation proposée. Les participants ont pu découvrir les recoins les plus secrets de la médiathèque. L'objectif de mêler, sans les opposer, outils numériques et missions traditionnelles de la médiathèque a été atteint naturellement.

Gage du succès, la complexité et la qualité de ce jeu n'ont absolument pas été perçues par le public, qui pensait probablement avoir affaire à de gentils animateurs assez doués pour la comédie...

L'équipe de l'espace jeunesse n'aura finalement que peu participé, étant donné la nouveauté du concept, et le manque de maîtrise des technologies employées. Nous étions en 2012, et découvrions tous les outils numériques mis en place. Une formation avait été effectuée en amont, mais le festival comportait pléthore d'actions, pour lesquelles les agents devaient s'impliquer, assurer une médiation peu simple : en 2012, personne ne savait ce qu'était une imprimante 3D, un kinect... L'équipe a, en amont du festival, assuré des présences hors-les-murs, dans les deux collèges de la ville, comme au centre commercial Ulis 2, et a entrepris, autour du numérique, une médiation nouvelle pour eux. J'étais moi-même peu sûre de mes 
explications lorsqu'il s'est agi, devant les caisses de Carrefour, d'expliquer le système du Friispray ou du piano invisible...

Cette action n'a pas été reconduite à l'identique, mais d'autres actions autour du jeu ont été mises en place de manière régulière (ateliers Minecraft notamment), nécessitant une préparation et un accompagnement moins lourds. Cependant, en 2013, les lapins Karotz ont été réutilisés lors d'un salon du livre : des lectures d'albums ont été enregistrées comme fichiers audio, et utilisées comme accompagnement ludique à la lecture pour les tout-petits.

On peut envisager d'organiser cette action de manière beaucoup moins coûteuse, en montant des partenariats avec des classes de collège, qui pourraient, avec leur professeur de français par exemple, écrire un scénario et le faire jouer par un club de théâtre. Enfin, l'utilisation ludique des lapins Karotz peut se concevoir en médiathèque pour présenter des expositions, et organiser des visites de manière ludique. 


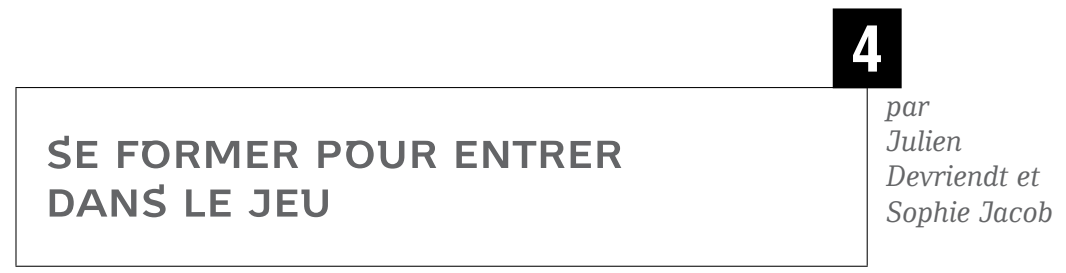

Jouer ne saurait être défini exclusivement par le faire, mais l'expérience englobe également l'être et l'attitude qui sous-tendent l'action : mission d'accompagnement délicate pour les équipes. De plus, au-delà des questions relatives à ses valeurs culturelles, éducatives et sociales, le jeu confronte également chacun à son parcours individuel et à sa propre relation avec le jeu.

Ainsi, quel que soit le degré d'intégration du jeu, ou du jouet, dans les établissements, qu'il soit perçu comme un simple produit de promotion pour la bibliothèque ou comme une offre à part entière, la formation des professionnels est indispensable.

Meilleure appréhension du domaine, initiation aux pratiques de l'animation avec le support-jeu, transfert de compétences, formations ciblées, journées d'études, partenariats, développement d'une expertise... autant de pistes de formation à suivre, selon les projets et l'environnement de chaque établissement.

Les ludothèques, partenaires historiques et essentiels, sont une ressource incontournable pour faciliter la mise en place de projets jeu dans les équipements qui ont la chance de pouvoir monter des partenariats de proximité. L'offre de formations s'est diversifiée ces dernières années et le panorama qui suit en recense les principaux acteurs. 


\section{OFFRES DE FORMATION POUR LE JEU ET/}

\section{ENCADRÉ OU LE JOUET : QUELQUES PISTES}

\section{CENTRES DE FORMATION SPÉCIALISÉS DANS LE JEU}

Le centre de formation aux métiers du jeu et du jouet (FM2J) ${ }^{1}$

Créé en 2006, à Lyon, le FM2J propose des formations à différentes catégories de professionnels, pour optimiser ou développer l'utilisation du jeu dans leur structure. L'offre couvre plusieurs secteurs : jeu et animation enfance/jeunesse, jeu et petite enfance, jeu et gérontologie, jeu et psychopédagogie, jeu et ressources humaines... En 2013, un nouveau secteur est ouvert : " culture et médiation par le jeu » qui propose une large palette de formations selon les besoins de chacun.

Une offre de stages courts

Sur 2 ou 3 jours pour acquérir ou consolider des savoir-faire professionnels :

1. Jeu et bibliothèque : comprendre l'intérêt d'implanter le jeu en bibliothèque. Faire cohabiter des espaces de jeu et des espaces de lecture ;

2. Le cadre ludique : découvrir les composantes du cadre ludique pour favoriser le jeu libre et autonome dans sa structure ;

3. Créer et gérer un fonds de jeux et jouets : constituer un fonds de jeux et jouets en fonction de son projet et de son public. Gérer et entretenir ce fonds ;

1. < http://www.fm2j.com/ > .
4. Médiation culturelle par le jeu : comprendre l'intérêt du jeu comme support de médiation. Acquérir les connaissances de base pour concevoir et mettre en œuvre des rencontres autour du jeu ;

5. Classement et analyse de jeux et de jouets : connaître et savoir utiliser les différents systèmes de classement existants (COL, ESAR). Faire des analyses critiques des jeux et jouets pour construire des propositions ;

6. Handilud : un stage transversal pour tous les professionnels souhaitant utiliser le jeu comme support d'inclusion des personnes en situation de handicap.

Des formations personnalisées

Parce que chaque structure, chaque équipe a un projet et un fonctionnement spécifiques, le centre construit des offres entièrement adaptées aux besoins, sur mesure. Toutes les formations se déroulent à FM2J ou dans les locaux du commanditaire.

Une formation au métier

de ludothécaire

Formation professionnelle certifiante permettant d'acquérir ou de valider les compétences nécessaires pour gérer une ludothèque ou un lieu de jeu et d'en assumer les responsabilités. La professionnalisation de ce métier encore jeune doit passer par la formation pour une pleine et entière reconnaissance. Elle se déroule sur 9 mois à raison d'une semaine par mois soit 350 heures. 


\section{Des conférences}

Exemples : Le jeu, médiateur incontournable pour le développement des $3^{\text {es }}$ lieux ; Le prêt de jeux et jouets, une démarche éco-citoyenne ? ; La ludothèque : son rôle dans la société contemporaine.

\section{Recherche}

Plusieurs actions de recherches ont déjà été menées et ont donné lieu à des outils pratiques pour les professionnels ${ }^{2}$.

Le secteur culture et médiation par le jeu alimente son expertise à partir d'un référentiel théorique toujours rattaché au terrain.

FM2J dirige actuellement la recherche Ludim $^{3}$, qui tend à démontrer l'efficacité du cadre ludique comme intervention non médicamenteuse de la maladie d'Alzheimer et contribue aux réflexions pour une sélection de jeux adaptés.

\section{Édition}

Documents, livres, catalogues, jeux et tous types de supports dont le thème principal est en rapport avec le jeu et les jouets ${ }^{4}$.

\section{L'Association des ludothèques fran- çaises (ALF) ${ }^{5}$}

L'ALF propose quelques stages de formation continue ${ }^{6}$, pas forcément en lien avec les bibliothèques, qui peuvent cependant être une ressource intéressante pour aborder des thématiques précises. Une carte interactive signale toutes les ludothèques sur le territoire, des rubriques sont dédiées aux ressources utiles aux ludothécaires et une liste de lieux de formations diplômantes est mise à jour - licences professionnelles, Master... ${ }^{7}$

L'ALF propose enfin un blog très actif : $<$ https://alfludotheques.wordpress.com/ >.

\section{Les ludothèques}

Elles proposent de plus en plus de formations et du conseil, ainsi que des animations pour les bibliothèques, assurant la transmission de leur savoir et savoir-être. Il est toujours intéressant de se rapprocher des ludothèques comme lieux ressources, conseils d'experts, sensibilisation au domaine.

2. «Des livres et des jouets, une cohabitation sur les espaces de la petite enfance est-elle possible ? ", étude menée en 2011, avec le soutien de la Caisse d'allocations familiales et du conseil général du Rhône. Le document est en accès libre sur le site des Presses de l'enssib, sous l'onglet Bonus, de cet ouvrage.

3. < http://www.fm2j.com/images/uploads/ revue-de-presse-jeu-et-gerontologie.pdf >.

4. Dernières publications à découvrir en ligne : < http://www.fm2j.com/ >.

5. < http://www.gestasso.com/association/ associationdesludothequesfrancaises/ $>$.

6. < http://www.gestasso.com/association/ associationdesludothequesfrancaises/lesformations-continues/ $>$.

7. < http://www.gestasso.com/association/associationdesludothequesfrancaises/creerune-ludotheque/ >. 
Quelques exemples de formations :

- Interlude ${ }^{8}$, Bordeaux : < http://www. ludotheque-interlude.fr/ $>$.

- Les enfants du jeu ${ }^{9}$, Saint-Denis :

$<$ http://www.les-enfants-du-jeu.com/ index.php $>$.

-HomoLudens, paysdel'Hérault:<http:// homoludensassocies.fr/v2/index.php/ les-ludotheques $>$.

- Ludomonde, Paris : propose des formations sur site, des prestations de conseils, des stages thématiques. Leur blog est très actif, une boutique en ligne offre une large gamme de jeux : $<$ http://www.ludomonde.coop/ >

\section{ORGANISMES DE FORMATION MÉTIERS DES BIBLIOTHÈQUES}

\section{Les différentes délégations du centre national de la fonction publique terri- toriale (CNFPT)}

Elles organisent régulièrement des formations autour du jeu faisant appel à des spécialistes du domaine. L'offre varie en fonction des régions, et aujourd'hui les formations portent essentiellement sur le jeu vidéo, peu encore concernent le jeu. On notera toutefois l'offre de la délégation Midi Pyrénées qui propose dans le cadre d'un itinéraire de formation plusieurs sessions sur la question du jeu en bibliothèque abordant l'aménagement des espaces, le système de classification

8. < http://www.ludotheque-interlude.fr/index-page-Formation.html $>$.

9. < http://www.les-enfants-du-jeu.com/formation_conseil_ludique_ile_de_france. php >.
ESAR et la coopération entre ludothèques et bibliothèques.

Même si votre délégation ne propose pas de formation dédiée au jeu, il est toutefois possible en fonction de vos projets de faire une demande de formation sur mesure pour votre établissement avec l'aide des conseillers formations du CNFPT. L'ensemble des formations du CNFPT est accessible surle site: $<$ http://www.cnfpt. $\mathrm{fr} /$ content/trouver-formation? $\mathrm{gl}=\mathrm{Nj}$ liOGJkMzI >.

\section{Les bibliothèques départementales de prêt}

Les BDP organisent régulièrement des stages de formation et des journées d'études.

Quelques exemples :

-BDP de l'Aude : < http://audealaculture.fr/formation-jeu-bibliotheque $>$;

- Médiathèque départementale de l'Hérault : < http://www.lr2l.fr/le-jeuen-bibliotheque.html > ;

- BDP de Lozère : < http://biblio. lozere.fr/index.php?option $=\mathrm{com}$ content\&id=342:jeux $>$.

\section{La Gaîté lyrique}

Lancé en 2013, « Gaîté formations » propose aux professionnels (entrepreneurs, artistes, techniciens, salariés en entreprise) des formations autour des cultures numériques. Elle propose aux bibliothécaires plusieurs formations autour du jeu vidéo : <http://gaite-lyrique.net/gaiteformations $>$. 


\section{Lecture Jeunesse}

En 40 ans d'expérience, Lecture Jeunesse est devenue l'association de référence sur la lecture des adolescents et des jeunes adultes. Depuis quelques années, elle propose aussi des formations autour du jeu vidéo et de la culture transmédiatique. Un numéro de la revue Lecture Jeune a été entièrement consacré à ce sujet ${ }^{10}$.

La BnF/Centre national de la littérature pour la jeunesse - la joie par les livres

La revue des livres pour enfants propose tous les deux mois une sélection de jeux vidéo et d'applications ludiques sur tablette réalisée par des bibliothécaires. En plus de la revue, une offre de for-

10. < http://www.lecturejeunesse.org/livre/lecture-jeune-149-mars-2014-les-jeux-video/ >. mations sur la médiation des ressources numériques et des jeux vidéo est également proposée : < http://lajoieparleslivres.bnf.fr/ >.

\section{L'Association des bibliothécaires de France (ABF)}

La commission Jeux vidéo de l'ABF a publié un ouvrage Jeux vidéo en bibliothèque et anime au quotidien un groupe de discussion professionnelle sur Facebook Jeux vidéo en bib : < https://www. facebook.com/groups/jvbib/ >. Chaque groupe régional organise également des journées d'étude.

Sur Facebook : un groupe de discussion professionnelle dédié au jeu est également ouvert à tous : < https://www.facebook.com/groups/j >. 


\section{MÉMENTO par Julien Devriendt}

\section{CONCEVOIR}

La mise en place d'une offre de jeu nécessite une réflexion dans le cadre $\mathrm{du}$ projet global de l'établissement. Elle interroge le fonctionnement du service public, l'organisation du travail interne et le rapport aux usagers. L'ensemble de l'équipe est ainsi concernée. Il va s'agir dans un premier temps de répondre ensemble à la question « En quoi proposer du jeu correspond aux missions de mon établissement ? ». Il sera alors important d'avoir une bonne connaissance du monde du jeu sous ses différentes formes en suivant des formations, en se rendant aux manifestations dédiées, en fréquentant des ludothèques et, bien sûr, en jouant.

Le champ est vaste et il sera difficile pour de nombreux établissements de proposer une offre globale de jeux pour tout public et sur tout support. N'hésitez pas cependant à concevoir votre projet de la manière la plus large possible, créez sur le papier votre projet idéal. Vos moyens humains, financiers sans oublier l'espace disponible se chargeront de vous rappeler la réalité. Au moins vous aurez une idée précise de ce vers quoi vos actions doivent tendre, vous permettant de ne pas vous enfermer dans vos propres représentations et d'aborder le jeu de manière transversale sans le cantonner à la seule section jeunesse.

Cette phase de préparation sera aussi l'occasion de s'assurer que l'ensemble du personnel a pris connaissance du projet d'établissement. Faites en sorte qu'il soit le plus clair possible et illustrez-le avec des actions concrètes déjà mises en place par votre équipe. Il sera plus aisé de penser globalement l'introduction de nouveaux services et cette formalisation favorisera les projets transversaux. Même si l'ensemble du personnel n'aura pas pour tâche d'animer un atelier autour du jeu ou de s'occuper du fonds dédié, tous seront impactés par la mise en place de ce nouveau service. Il sera également plus facile d'argumenter et de défendre le projet auprès des élus, des équipes, collègues et usagers. 
Insistez sur les outils d'évaluation, vos projets vont connaître des formes différentes, vous devrez vous adapter, réaménager l'espace, modifier vos animations. Il n'existe pas une seule solution, Inspirez-vous de fonctionnements, d'aménagements d'autres établissements pour les adapter à votre structure. Il est important de documenter et de partager les projets mis en place, cela vous permettra de garder un historique de l'évolution de vos projets, les nouveaux arrivants au sein de votre équipe vous en seront reconnaissants.

Nous diffusons déjà sur notre territoire notre programme d'animation auprès de nos partenaires, partagez également vos formats d'ateliers, vos ressources, avec les ludothèques, écoles, centres d'animation, associations, etc. Mettez en place un site collaboratif, comme un wiki par exemple, pour échanger vos idées, publiez vos fiches d'ateliers. Cela ne remplace pas les visites auprès de vos partenaires mais permettra de valoriser sur le territoire les actions déjà mises en place et suscitera de nouveaux partenariats.

D'un point de vue technique, la plupart des supports de jeux ne poseront pas de difficultés. Toutefois pour le jeu vidéo, il est indispensable de négocier en amont avec les services informatiques un accès au réseau permettant de mettre à jour facilement le matériel (tablettes, consoles) et de télécharger des jeux dématérialisés au risque de vous couper d'une part importante de la production vidéo ludique.

\section{Les 5 questions fondamentales}

Pourquoi ? En quoi cette action répond aux missions de mon établissement?

Quel public?

Quels moyens ? Humains, matériels, financiers

Quel espace?

Quels partenaires? 


\section{METTRE EN CEUVRE}

\section{SE FORMER ET ACCOMPAGNER LE PERSONNEL}

Composez un groupe de travail représentant différentes sections de votre organigramme, comprenant à la fois des joueurs et des non joueurs, et si possible un référent professionnel du jeu. Le groupe de travail sera chargé de transcrire les grandes intentions du projet d'établissement en propositions d'actions concrètes, de l'animation ponctuelle au projet de réaménagement de votre établissement.

Il va être nécessaire de former les collègues aux univers des jeux pour établir des points de connexion avec les autres collections mais aussi pour découvrir leurs spécificités. Pouvoir s'appuyer sur des membres de l'équipe qui jouent déjà à titre personnel est un atout non négligeable. Cela passe à la fois par des séances sur le temps de travail, au même titre qu'une formation interne, que par la mise à disposition de jeux auprès du personnel, pour jouer sur place ou sur son temps libre.

Une partie de la formation pourra être assurée en interne si vous disposez des recrutements nécessaires, toutefois les formations à l'extérieur permettront des temps de prise de recul nécessaires à la conception de votre projet.

\section{ACQUÉRIR}

Il est tentant de se focaliser uniquement sur le choix du jeu à proposer au public. Toutefois, les acquisitions se font dans le cadre de la politique d'acquisition de l'établissement. Cette dernière, tout comme le projet d'établissement, pourra faire l'objet d'une adaptation. Le choix des acquisitions se fera au croisement des objectifs de l'établissement, des besoins du public, des contraintes matérielles, humaines et financières ainsi que de l'offre déjà existante sur le territoire.

Un jeu se gère de la même manière qu'un livre ou tout autre support, une veille est à mener sur le sujet pour se tenir au courant de l'actualité au travers de la presse spécialisée, des sites web dédiés ou en vous rendant à des salons. Vous pourrez également trouver de nombreux conseils 
en boutiques spécialisées. Avec un peu de temps vous trouverez vite vos repères parmi les éditeurs et les différents prix décernés chaque année.

\section{CLASSIFICATION}

Pour le catalogage, en fonction de vos besoins, vous devrez adapter votre modèle de notice aux différents supports de jeux. Pour la cotation et l'indexation, deux systèmes existent, comparables au système Dewey, l'ESAR ou le classement des objets ludiques (COL). En fonction de votre projet vous pouvez vous en inspirer pour développer votre propre système de classement. Tout dépendra de votre projet et de votre mode de fonctionnement. Le détail complet des boîtes de jeu en note peut se révéler fastidieux mais reste indispensable si vous souhaitez prêter les jeux.

\section{ÉQUIPEMENT}

Certaines ludothèques et bibliothèques impriment le contenu de la boîte pour le coller dans le couvercle. Si vous ne proposez pas de prêt cela peut sembler superflu, toutefois nous ne sommes jamais à l'abri d'une pièce perdue. Une carte en moins sur un jeu de question réponse ne pose pas de difficultés, à l'inverse, certains jeux peuvent se révéler injouables s'il leur manque un élément. À vous donc de fixer vos règles. Un règlement intérieur permettra de cadrer l'utilisation des jeux sur place et aidera le personnel à tenir un discours commun.

Dans le cadre d'une offre de prêt, il faut donc vérifier l'ensemble des pièces, au retour ; pour cela, certaines équipes utilisent une balance de précision. Dans tous les cas prévoyez des pièces de rechange.

\section{ANIMATIONS PONCTUELLES}

N'hésitez pas à commencer petit, il sera plus facile pour votre équipe de se faire la main sous la forme d'animations ponctuelles, comme des soirées jeux à destination des adultes, dans un premier temps, pour mettre en place de nouveaux partenariats et tester l'appétence du public.

Ces premières animations ne seront pas toutes couronnées de succès, de nombreux éléments seront certainement à revoir comme l'aménagement 
du lieu, le choix des jeux, la façon de les présenter, d'organiser (ou non) les temps de jeu, de gérer la circulation des joueurs, etc. Un temps d'adaptation sera nécessaire, certaines idées seront abandonnées en cours de route.

Pas besoin d'être spécialiste sur l'ensemble des jeux du marché, faites-vous aider en invitant les communautés de joueurs à co-organiser des événements et faire découvrir leurs passions. Mettez à disposition vos ressources matérielles, documentaires mais aussi vos compétences. Le partage de l'information, la coopération sont au cœur même du succès de jeux comme Minecraft. S'appuyer sur une pratique motivante pour initier aux règles d'écriture sur un wiki, documenter l'avancement de son projet de construction sur son blog, créer un Machinima ${ }^{1 *}$ (film d'animation réalisé à partir de captures vidéo du jeu), développer un mode de jeu spécifique ou créer des objets en 3D sont autant d'occasions de développer des usages créatifs. Faites-vous connaître, soyez présent sur les différentes manifestations autour du jeu de votre territoire, vos missions ne s'arrêtent pas aux portes de votre établissement. Le wiki mis en place lors de la phase conception du projet se révélera ici un excellent outil de médiation auprès de vos partenaires.

\section{CRÉEZ VOTRE JEU}

Vous pouvez également vous appuyer sur des dispositifs ludiques pour favoriser la participation, les échanges avec les lecteurs. Sans aller jusqu'à la mise en place d'un jeu en réalité alternée (ou Alternate Reality Game), vous pouvez proposer des parcours différents au sein de vos espaces par la mise en place de chasse au trésor, d'enquête policière ou de challenge à réaliser, mettant en scène le personnel et les collections. Pour les plus ambitieux, la création d'un jeu d'envergure va nécessiter la mise en place de partenariats ou de collaborations avec des sociétés spécialisées. La médiathèque des Ulis avait ainsi fait appel à la société XiLabs en février 2011 pour la création d'un jeu géolocalisé sur la ville, appelé Love Quest, auquel les joueurs pouvaient accéder à partir de leur smartphone.

1. Compte rendu d'un atelier Machinima à l'Astrolabe de Melun : < http://www.astrolabe-melun. fr/astrolabe-melun.fr/cms/articleview/id/776 >. 


\section{JOUER SUR PLACE}

Au-delà du règlement intérieur, c'est l'aménagement qui permettra de réguler l'utilisation de l'espace. En fonction des jeux prévoyez un mobilier adapté et autant de chaises que de joueurs. Certains jeux sont bruyants, d'autres au contraire nécessitent calme et concentration, ne regroupez pas tous les jeux au même endroit.

La manière dont le public sera amené à évoluer au sein de l'espace, la disposition des espaces de jeux, la présentation et l'accessibilité des jeux inciteront plus ou moins le public à s'approprier les lieux et à en respecter le cadre. Même en cas d'espace séparé, essayez de faire en sorte qu'il puisse rester visible. De nombreux changements seront nécessaires avant de trouver un équilibre. Une fois trouvé, celui-ci reste précaire, l'introduction d'un nouveau jeu nécessitant plus de place pourra impacter le reste de l'espace.

\section{LA POSTURE DU PERSONNEL}

Le personnel, en fonction de la situation, pourra adopter plusieurs postures :

- le public s'est emparé des jeux mis à disposition et s'est lancé dans une partie, rien ne leur manque. Le personnel reste à distance et est garant du cadre du jeu. Le travail se situe en amont à travers l'aménagement du cadre du jeu et l'accompagnement des parents pour les plus jeunes ;

- le jeu est inconnu des joueurs, la partie ne démarre pas, le personnel va aider la partie à se lancer en allant présenter le jeu, en faisant une ou deux parties pour rien. Il pourra en fonction du déroulement de la partie quitter le jeu, proposer à une autre personne de rejoindre la partie ou rester jouer.

La mise à disposition de jeux pour une consultation sur place ou lors d'une animation implique que le personnel connaisse les règles du jeu pour aider le public à lancer la partie si besoin, soit en expliquant succinctement les règles, soit en jouant lui-même pour que la partie puisse avoir lieu. Il est donc nécessaire qu'il puisse jouer sur son temps de travail et que cela soit inscrit sur sa fiche de poste. 


\section{GLOSSAIRE}

Alternate Reality Game

Voir jeu en réalité alternée.

\section{Balance de précision}

Plutôt que de recompter les pièces, les ludothécaires vérifient le poids des boîtes à l'aide d'une balance de manière à ne pas recompter les pièces systématiquement.

\section{Borne d'arcade}

Voir salle d'arcade.

\section{Catalogue ludifié}

Catalogue de bibliothèque disposant de fonctionnalités débloquant des récompenses (badges, points, etc.) en fonction de l'activité des usagers : nombre d'emprunts, commentaires/critiques déposés, partages sur les réseaux sociaux. L'obtention d'un certain nombre de points peut donner droit à des privilèges spécifiques (choix sur les acquisitions et/ou la programmation, emprunt en avant-première, etc.) L'objectif étant d'inciter les usagers à créer du contenu sur le catalogue et le diffuser.

\section{Communauté}

Dans le cadre d'une communauté d'intérêt ou de connaissance, regroupement d'acteurs autour d'une question, d'un intérêt partagé, échangeant leurs connaissances et savoir-faire via des outils de communication numériques et/ou lors de rencontres physiques en vue de les formaliser pour leurs besoins particuliers.

\section{Concours de création}

Ces rencontres permettent aux créateurs (jeux de société et jeux vidéo) de présenter leur jeu ou prototype au public et aux professionnels en vue de se faire éditer. Une variante, issue du milieu du jeu vidéo, appelée game jam consiste à réaliser un jeu sur un thème imposé dans un temps imparti.

\section{Conditions générales d'utilisation (CGU)}

Document contractuel encadrant les relations entre un service et l'utilisateur. Dans le cas des jeux vidéo, il s'agit d'un document à valider lors de la première mise en route d'une console ou d'un logiciel et lors d'une mise à jour.

\section{Cosplayer}

Issu du mot valise cosplay composé des termes anglais costume et play. Il s'agit d'une activité consistant à jouer le rôle d'un personnage issu le plus souvent d'un manga, d'un jeu vidéo ou de comics, en reprenant son apparence et son attitude. D'abord popularisée par les fans de Star Wars et Star Trek aux États-Unis, la pratique s'est exportée au Japon et en 
Europe notamment lors de salons dédiés au manga ou aux jeux vidéo. La création originale de costume est très valorisée parmi les copslayers, des concours sont également organisés pour récompenser les meilleurs d'entre eux.

\section{Créateur de jeux}

Voir Game designer.

\section{Éditeur de niveaux}

Fonctionnalité offerte par certains jeux permettant de modifier l'agencement des éléments du jeu (ennemis, obstacles, bonus, etc.) pour y faire ensuite évoluer son personnage. Certains jeux vidéo proposent de partager les créations des joueurs via Internet renouvelant ainsi l'intérêt des joueurs qui accèdent à de nouveaux contenus.

\section{Expérience de jeu ou gameplay}

Ensemble des éléments qui vont affecter le ressenti du joueur vis-à-vis d'un jeu : complexité des règles, la manière d'évoluer au sein de l'environnement, les possibilités offertes et les contraintes imposées, le niveau de difficulté. L'ensemble de ces éléments proposera une expérience de jeu que l'on pourra qualifier de plus ou moins réussie et/ou originale.

\section{Game designer ou créateur de jeux}

Responsable de la conception du jeu, il va être chargé de définir le but du jeu et les étapes pour y parvenir. Il va donc élaborer l'ensemble des règles de fonctionnement et les possibilités offertes au joueur qui définiront l'expérience de jeu à atteindre. Même s'ils ne sont pas les seuls à décider de la direction du jeu, certains game designers bénéficient d'un véritable statut d'auteur.

\section{Game-documentaire $^{1}$ ou newsgame}

Se basant sur un fait d'actualité, le newsgame propose de placer le joueur au cœur d'une situation complexe en lui proposant de tester différentes combinaisons. Un game over ne signifie pas un échec mais la possibilité de tester une autre approche, permettant au joueur de mieux comprendre des événements complexes en les expérimentant lui-même.

\section{Gameplay}

Voir expérience de jeu.

\section{Gamification}

Voir ludification.

\section{Installation $\mathrm{du}$ jeu}

Pour les jeux de société, c'est le fait de mettre en place les différents éléments $\mathrm{du}$ jeu et s'assurer que l'ensemble des éléments dont les joueurs auront besoin sont bien présents. Pour les jeux vidéo, il s'agit de l'installation du jeu sur le disque dur de la machine.

1. Voir la contribution de Julien Devriendt, "Silence on joue ! Le médiateur, les jeux vidéo et les fonds documentaires ", in Xavier Galaup (dir.), Développer la médiation documentaire numérique, Villeurbanne, Presses de l'enssib, 2012 (coll. La Boîte à outils ; 25), pp. 168-172. 
Jeu d'aventure de type point and click

Jeu vidéo se contrôlant entièrement à l'aide de la souris. Le personnage incarné par le joueur devra se déplacer de tableau en tableau pour résoudre des énigmes à l'aide d'éléments du décor qu'il devra découvrir, puis éventuellement combiner. Très populaire dans les années 1990, ce genre connaît depuis quelque temps un regain d'intérêt avec notamment les adaptations de comics comme The Walking Dead ou Fable, The Wolf among us, par le studio Telltale.

\section{Jeu du monde}

Appellation qui renvoie vers des jeux traditionnels de différents pays, par exemple le Surakarta (Indonésie) et l'Awalé (Afrique).

\section{Jeu en réalité alternée ou Alternate Reality Game}

Jeu se déroulant dans le monde réel via différents moyens de communication : livres, prospectus, affiches, mails, blog, vidéo, forum, etc. Des indices sont laissés par les organisateurs incitant les participants à suivre différentes pistes et tenter de résoudre des énigmes. Le jeu se déroule en temps réel renforçant un peu plus le sentiment d'immersion.

\section{Jeu sérieux (ou serious game)}

"Application informatique, dont l'objectif est de combiner à la fois des aspects sérieux (serious) tels, [...], l'enseignement, l'apprentissage, la communication, ou encore l'information, avec des ressorts ludiques issus du jeu vidéo (game) $»^{2}$.

\section{Jeux d'action - jeux d'aventure}

Mêlant à la fois des éléments des jeux d'action et des jeux d'aventure, alternant des phases d'exploration de résolutions d'énigme et de combat, le genre sollicite à la fois l'habileté, la dextérité et la rapidité des joueurs tout en offrant un scénario, des dialogues et un système d'inventaire.

\section{Survival - horror}

Généralement en vue à la troisième personne, le joueur doit survivre dans un environnement hostile rempli de monstres, zombies, animaux enragés. Le genre utilise tous les codes du cinéma d'horreur pour créer des ambiances oppressantes.

\section{Infiltration}

Jeux où l'objectif premier est de ne pas être repéré et d'éviter au maximum les conflits pour s'introduire au cœur d'une base ennemie. Le joueur devra observer les habitudes des gardes, utiliser l'environnement à son avantage pour s'y dissimuler, créer des diversions.

2. Définition issue de Julian Alvarez, Du jeu vidéo au Serious Game : approches culturelle, pragmatique et formelle, Thèse spécialité science de la communication et de l'information, sous la direction de JeanPierre Jessel et Gilles Methel : Toulouse, université de Toulouse II (Le Mirail) ; université de Toulouse III (Paul Sabatier), décembre 2007, p. 9. 


\section{Jeux d'ambiance}

Jeux faciles d'accès caractérisés par des règles simples, des parties relativement courtes et nécessitant peu de matériel. Les jeux d'ambiance insistent davantage sur les relations entre les joueurs, à travers notamment des jeux de mémoire, de questions réponses ou de rapidité.

\section{Jeux de combat ou Versus Fighting}

Jeux dans lesquels deux personnages s'affrontent dans une arène fermée. L'adversaire peut être contrôlé par un autre joueur ou le jeu lui-même. Le combat est vu de profil, les personnages se faisant face de chaque côté de l'arène. Il est ainsi possible à deux joueurs de s'affronter sur un même écran. Les joueurs ont la possibilité de choisir parmi plusieurs personnages ayant chacun des caractéristiques et des aptitudes différentes.

\section{Jeux de plate-forme}

Jeux dans lesquels le personnage doit traverser un niveau en sautant d'une plate-forme à une autre en franchissant des obstacles et en évitant les ennemis ou en s'en débarrassant.

\section{Jeux de rôle ou Role Playing Game (RPG)}

Descendant des jeux de rôle papier, ce gameplay est basé sur le développement des personnages. Le joueur crée son avatar en choisissant une catégorie moine, magicien, soldat... Par un système de points d'expériences, le joueur pourra, à la fin des combats, faire évo- luer son personnage et débloquer de nouvelles compétences.

\section{Jeux de rôle tactique \\ ou Tactical Role Playing Game}

Mélange entre le jeu de rôle et le jeu de stratégie classique. Le joueur contrôle plusieurs personnages. Les combats se déroulent au tour par tour sur une carte découpée en cases sur lesquelles les unités se déplacent.

Jeux de rôle en ligne massivement multi-joueur ou Massively Multiplayer Online Role Playing Game (MMORPG)

Jeux de rôle dans lesquels les joueurs font évoluer leur personnage au sein d'un univers persistant ; c'est-à-dire qu'il continue à évoluer même lorsque le joueur n'est pas connecté. Des communautés de joueurs se sont constituées pour s'entraider, s'échangeant ressources, matériel et réalisant ensemble les différentes quêtes du jeu.

Jeux de type casse-tête ou énigmes Généralement basé sur une idée de $g a-$ meplay simple qui sera ensuite déclinée pour en augmenter la difficulté au fur et à mesure de l'avancement dans le jeu.

Jeux de stratégie au tour par tour Chaque camp dispose de temps pour planifier son action en fonction du placement de son adversaire. Une fois les actions validées, c'est au tour de l'adversaire. Les joueurs planifient leurs actions 
sur plusieurs tours donnant lieu à des parties souvent très longues.

Jeux de stratégie en temps réel ou Real Time Strategy (RTS)

Le joueur, en plus de ses capacités de réflexion, devra faire preuve de réactivité pour ne pas se laisser déborder par son adversaire. Il devra gérer simultanément la collecte des ressources, le déplacement de ses unités et les combats.

Jeux de gestion

Le joueur se place dans le rôle d'un gestionnaire (maire, directeur, devant construire, développer et gérer une ville, une entreprise, un hôpital, un parc d'attractions, etc.). Le joueur doit utiliser les ressources mises à sa disposition (impôts, revenus, etc.) pour développer de nouveaux services et obtenir des ressources supplémentaires.

\section{Jeux de rythme ou Rhythm based Game}

Jeux basés sur la dextérité du joueur à suivre le rythme d'une chanson. Les joueurs doivent suivre en rythme une série de commandes apparaissant à l'écran à l'aide d'une manette ou de contrôleurs particuliers comme un tapis de danse ou des guitares en plastique.

\section{Jeux de tirs en vue subjective ou First Person Shooter (FPS)}

Jeux dans lesquels le joueur voit la scène à travers les yeux de son personnage ne laissant apparaître que les mains et les armes. Les FPS sont pratiqués lors de tournois internationaux où plusieurs équipes s'affrontent.

\section{Jeux surdimensionnés}

Jeux traditionnels en bois tels que les jeux de palets ou de lancer d'anneaux. Rentrent également dans cette catégorie les adaptations grand format de jeux de société classiques (Mikado, échecs, etc.).

\section{Licence légale}

La licence légale, dans le cadre du droit de prêt des livres en bibliothèque, est une autorisation prévue par la loi permettant aux établissements de prêter les livres de leur collection en échange d'un mécanisme de compensation financière en direction des ayants droit.

\section{Livre-jeu ou game book}

Romans interactifs ou livres dont vous êtes le héros, dont le déroulement dépendra des choix des joueurs. Il peut également s'agir de livres-jouets surtout destinés à la petite enfance souvent composés d'éléments à déplacer ou de puzzle.

\section{Ludification ou gamification}

Consiste à transférer des mécanismes de jeu dans d'autres domaines afin d'améliorer l'expérience des utilisateurs. Dans les domaines de l'apprentissage, de la santé, l'objectif est d'inciter l'usager à effectuer des tâches en les rendant plus agréables et/ou acceptables. 


\section{Machinima}

Mot valise issu de la contraction des termes machine et animation, il s'agit de films d'animation réalisés à partir de captures vidéo de jeux vidéo. Les créations sont ensuite diffusées sur le Web. Des concours sont organisés chaque année récompensant les meilleures créations.

\section{Mécanique de jeu}

Voir gameplay.

\section{Mini-jeu d'arcade}

Voir salle d'arcade.

\section{Mise en jeu}

Ensemble des actions mises en place par le médiateur (bibliothécaire ou ludothécaire) afin de proposer et de permettre la découverte d'un jeu au public. De l'aménagement de l'espace à la présentation des règles jusqu'au lancement et suivi de la partie en fonction de l'âge et de l'expérience des participants.

\section{Newsgame}

Voir game-documentaire.

\section{Principe du « trou de lapin »}

Dans le cadre d'une approche transmédia, on désigne par le terme "Rabbit Hole" ou « trou de lapin » une porte d'entrée par laquelle un spectateur, un internaute ou un fan peut entrer dans l'univers narratif développé par le storyteller. Ce peut être un site web, un email, une page Facebook ou encore l'élément central du dispositif comme la série ou le film.

Voir "the Rabbit Hole", lexique. [En ligne] : < http://www.therabbithole.fr/lexique/ >.

\section{Pure player}

Désigne une entreprise dont l'activité se déroule exclusivement sur le Web.

\section{Rejouabilité}

Intérêt pour le joueur de recommencer une partie une fois le jeu terminé.

\section{Rétrogaming}

Pratique consistant à jouer à des jeux vidéo datant des années 1970 à 1990 sur les consoles d'origine ou sur PC via des émulateurs. Plusieurs associations regroupant passionnés et collectionneurs organisent des salons et proposent des animations en bibliothèque.

\section{Salle d'arcade ou borne d'arcade, mini-jeu d'arcade}

Très populaires au début des années 1990, salles de jeux comprenant des jeux vidéo sur bornes avec monnayeurs.

\section{Shoot 'em up}

Que l'on peut traduire par « détruisezles tous ». Il s'agit de jeux où le joueur contrôlant un véhicule doit tirer à un rythme effréné sur des vagues d'ennemis. Les jeux sont découpés en niveaux avec une difficulté croissante. 


\section{Third Person Shooter (TPS)}

Semblables aux FPS, les TPS sont des jeux de tirs à la troisième personne (ou tir objectif) dans lesquels le personnage contrôlé apparaît à l'écran vu de dos.

\section{Transmission des règles}

Avant de pouvoir expliquer la règle d'un jeu et lancer la partie, le médiateur devra s'approprier le jeu, décou- vrir l'univers proposé et identifier les mécanismes ludiques. Ainsi en fonction de l'âge, de l'expérience et du nombre de joueurs, il pourra adapter sa présentation pour que la découverte du jeu soit la plus accessible possible. 


\section{BIBLIOGRAPHIE}

Julian Alvarez, Du jeu vidéo au serious game: approches culturelle, pragmatique et formelle, Thèse spécialité science de la communication et de l'information, sous la direction de Jean-Pierre Jessel et Gilles Methel : Toulouse, université de Toulouse II (Le Mirail) ; université de Toulouse III (Paul Sabatier), 2007.

Roger Caillois, Les jeux et les hommes : le masque et le vertige, édition revue et augmentée, Paris, Gallimard, 1967 (coll. Idées ; 125).

Hélène Dres, Le jeu et la bibliothèque : un outil pour changer d'image ou un réel changement ?, Mémoire de Master 2 Sciences humaines et sociales, mention Sciences de l'information et de la communication, sous la direction de Marie-Christine Jacquinet : Nanterre, université Paris Ouest Nanterre La Défense, 2010. [En ligne] : < http:// www.enssib.fr/bibliotheque-numerique/documents/49083-le-jeu-et-labibliotheque-un-outil-pour-changer- $d$ image-ou-un-reel-changement.pdf >.

Denise Garon, Rolande Filion et Robert Chiasson (collab.), Le système ESAR : guide d'analyse, de classification et d'organisation d'une collection de jeux et jouets, Montréal, ASTED ; Paris, Éditions du Cercle de la Librairie, 2002.
Marie Latour, La ludification en bibliothèque : utiliser le jeu comme médium dans la relation aux usagers, Mémoire d'étude de conservateur des bibliothèques, sous la direction de Guillemette Trognot : Villeurbanne, enssib, 2014.

Françoise Legendre, Jeu et bibliothèque : pour une conjugaison fertile, Rapport n 2015-009 de l'Inspection générale des bibliothèques, février 2015, pp. 89-90. [En ligne] : < http://www.enssib.fr/bibliothequenumerique/documents/65198-jeu-etbibliotheque-pour-une-conjugaisonfertile.pdf $>$.

Cécile Meneghin, Des jeux vidéo à la bibliothèque, Mémoire d'étude de conservateur des bibliothèques, sous la direction de Benoît Epron : Villeurbanne, enssib, 2009. [En ligne] : < http://www.enssib.fr/bibliothequenumerique/documents/2102-des-jeuxvideo-a-la-bibliotheque.pdf > .

Scott Nicholson, Everyone Plays at the Library: Creating Great Gaming Experiences for All Ages, Medford, Information Today, 2010.

Odile Périno, Des espaces pour jouer : pourquoi les concevoir ? Comment les aménager?, nouvelle édition entièrement revue et augmentée, Toulouse, Erès, 2014 (coll. Enfance et parentalité). 
Revue Hermès, 2012, $\mathrm{n}^{\circ} 62$ "Quand jouer, c'est communiquer ». [En ligne] : < http://www.cairn.info/revue-hermesla-revue-2012-1.htm >.
Anne-Gaëlle Gaudion et Nicolas Perisse (dir.), Jeux vidéo en bibliothèque, Association des bibliothécaires de France, 2014 (coll. Médiathèmes). 


\section{INDEX DES JEUX ET JOUETS}

TyPe DE JEU

Chasse au trésor 14, 123, 142, 144, 157

Console Wii 9, 90

Jeu « bac à sable » 98

Jeu collaboratif 70

Jeu coopératif 92

Jeu d'ambiance 91, 92

Jeu d'assemblage 58-61

Jeu d'aventure de type point and click 97

Jeu d'esprit 17

Jeu d'éveil 58

Jeu déveil sensoriel 92

Jeu d'exercice 58-61

Jeu d'osselets 16

Jeu de cartes 16-17, 20-21, 23, 58, 117

Jeu de cases 16

Jeu de casse-tête 90

Jeu de construction $67,90,92$

Jeu de coopération 90

Jeu de courses 16

Jeu de dés 16, 58

Jeu de figurine 21, 43

Jeu de Go 16

Jeu de logique 90

Jeu de loterie 17, 23

Jeu de manipulation 58

Jeu de miroir 58

Jeu de mot 17, 23

Jeu de plateau $10,17,21,23,43,58,90,100$, 104, 117

Jeu de plein air 90

Jeu de puzzle 85,124

Jeu de règles $58-61,65,67,82,116$

Jeu de rôle $21,23,34,54,58,60-61,82$,

85,90

Jeu de semailles 16

Jeu de société 10, 19-21, 32-35, 39-43, 46,

$56-59,61,90,92,111,116,118,127$

Jeu du monde 91-92
Jeu éducatif $34,81,90$

Jeu en ligne 45, 54, 70, 90, 123

Jeu en réalité alternée 13, 128, 131, 135, 157

Jeu en réseau 90, 105-107

Jeu géant 90

Jeu historique 21

Jeu interactif 90

Jeu multi-joueurs 54,91

Jeu physique $42,44,46,94$

Jeu porte-monstre-trésor $85-86$

Jeu sérieux 53, 69-70, 76-77, 79-84, 87-88, 122

Jeu social 132

Jeu sur tablette 94

Jeu surdimensionné 91-92

Jeu symbolique $11,17,58-61,64-67$

Jeu transmédia 131

Jeu vidéo 10-11, 17, 19, 21, 24, 31-32, 34-37, $39,41-43,45-48,50-53,56-58,61-$ 63, 69-70, 77-78, 80-83, 87, 97-98, 100-105, 108-109, 111, 113, 122, $127,131,150-151,154$

Jeu vidéo " bac à sable " 104 Jeu vidéo de construction virtuelle 61 Jeux personnels 70

Serious game 12, 71, 77, 137

\section{NOM DE JEUX}

A Google a Day 84

A Journey through Europe 20

Adibou 81

Alternate Reality Game 131, 157

America's Army 82

Another World 51

Ayiti: The Cost of Life 82

Citadelles 20

Civilization 81

Civilization III 81

Cluedo ${ }^{\circledR} 59$ 
Counter strike 116

Defense of the Ancients 98

Destiny 82

Dishonored 51

Dixit 23

Dofus 54

Escouade B 12, 79-88

Find The Future: The Game 13, 125

Foldit 124

Food Force 82

Fort Mc Money 70, 75

Grand Theft Auto 40

Guerre des moutons (La) 20

Halli Galli 116

Hordes 54

ICO 51

Il était une fois 23

Ingress 123

Linq 23

Loups-garous de Thiercelieux (Les) 20

Love Quest 157

Mac Donald's 70-71

Mais où est Carmen Sandiego ? 81

Mario Party 116

Mario Teaches Typing 81

Memory 114

Metal Gear 51

Minecraft 98, 102-104, 106-109, 157

Monkey Island 97

Monopoly 20,59

Mush 54

Pictionnary 59

Res Publica 20

Schotten-Totten 20

Shadow of the Colossus 51
Senet 16

Sim City 81

Speech 23

Street Fighter 2x 99

Sudoku 116

Tamagocours 70, 73-74

The Last of Us 51

Wakfu 54

Warcraft III 98

Weiqi 16

Zombie Division 70, 73

\section{JouET}

Château fort 67

Circuit 64

Déguisement 64, 67

Dînette 67

Dinosaure 67

Duplo 67

Ferme 67

Figurine 58, 64, 67

Jouet 10-11, 17, 19, 56, 58, 61, 64-67, 111, $113,116,118$

Jouet d'imitation 64

Lego ${ }^{\circ} 58,67,104$

Lego Technic ${ }^{\oplus} 67$

Livre-jeu 23

Kapla 58, 67

Maison de poupées 67

Pompier 67

Poupée 64, 67

Train 67

Voiture 67 


\section{LISTE DES AUTEURS}

\section{Véronique Amar}

Assistante principale de conservation, secteur numérique, bibliothèque du $9^{\mathrm{e}}$ Duchère, Bibliothèque municipale de Lyon

\section{Anthony Avila}

Responsable multimédia - ludothèque - informatique, Médiathèque d'Osny

\section{Florence Carre}

Directrice des affaires culturelles de la Communauté d'agglomération du Plateau de Saclay

\section{Julien Devriendt}

Responsable numérique - Médiathèque Aragon - Choisy le Roi

\section{Catherine Di Sciullo}

Responsable du Centre de ressources de la Gaîté lyrique (Paris)

\section{Thomas Fourmeux}

Assistant multimédia, Bibliothèques d'Aulnay-sous-Bois, animateur du blog Biblio Numericus

\section{Sophie Jacob}

Formatrice et chercheuse associée du Centre de formation aux métiers du jeu et du jouet FM2J (Lyon)

\section{Caroline Makosza}

Responsable de la ludo-médiathèque de Fosses

\section{Lionel Maurel}

Chargé de mission Internet \& nouvelles technologies, Bibliothèque de documentation internationale contemporaine (Nanterre)

\section{Céline Meneghin}

Directrice de la bibliothèque départementale de la Somme

\section{Thierry Robert}

Bibliothécaire, Bibliothèques de Montréal

\section{Éric Sanchez}

Maître de conférences à l'Institut français de l'éducation (École normale supérieure de Lyon)

\section{Guillemette Trognot}

Documentaliste, Bibliothèque Marie Curie, INSA Lyon

\section{Marie-Paule Voïta}

Documentaliste, Bibliothèque Marie Curie, INSA Lyon

\section{Solenne Waszak}

Responsable du pôle Action culturelle de la médiathèque départementale $d u$ Puy-de-Dôme

\section{Élise Ybled}

Responsable du pôle Multimédia (informatique, jeux vidéo, de société et de rôle), médiathèque Blaise-Cendrars, Conflans-Sainte-Honorine 


\section{LA BOÎTE À OUTILS}

Fondée par Bertrand Calenge en 1995.

Les manuels de cette collection visent à fournir aux professionnels des ouvrages pratiques pour conduire des projets bibliothéconomiques d'actualité concernant aussi bien des bibliothèques publiques que des bibliothèques universitaires ou de recherche. Écrit à plusieurs mains, chaque volume est coordonné par un professionnel des bibliothèques.

Catherine Jackson

directrice de la collection
$+++++++++++++++++++++$

\section{PRESSES DE L'enssib}

École nationale supérieure des sciences de l'information et des bibliothèques

17-21 boulevard du 11 novembre 1918 69623 Villeurbanne Cedex

Tél. 0472444343

Fax 0472444344

< http://www.enssib.fr/presses >
Dans la même collection, à paraître

BA0 \#35 (2015)

Médiatiser la science en bibliothèque sous la direction de Justine Ancelin

Déjà parus

$++++++++++++++++++++++++++++++++++++$

BAO \#33 (2015)

Construire des pratiques participatives

dans les bibliothèques

sous la direction de Raphaëlle Bats

BA0 \#32 (2015)

Conduire le changement

en bibliothèque : vers

des organisations apprenantes

sous la direction de Christophe Pérales

BA0 \#31 (2014)

Ouvrir plus, ouvrir mieux :

un défi pour les bibliothèques

sous la direction de Georges Perrin

BA0 \#30 (2014)

Produire des contenus documentaires en ligne: quelles stratégies pour les bibliothèques? sous la direction de Christelle di Pietro

BA0 \#29 (2014)

Intégrer des ressources numériques dans les collections

sous la direction de Géraldine Barron et Pauline Le Goff-Janton

BA0 \#28 (2013)

Favoriser l'insertion professionnelle et l'accès à l'emploi : les atouts des bibliothèques sous la direction de Georges Perrin

BA0 \#27 (2012)

Faire connaître et valoriser sa bibliothèque : communiquer avec les publics sous la direction de Jean-Marc Vidal 
Déjà parus

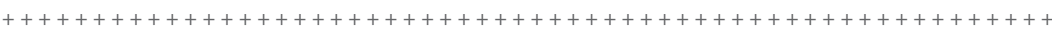

BA0 \#26 (2012)

Apprendre à gérer des collections patrimoniales en bibliothèque sous la direction de Dominique Coq

BA0 \#25 (2012)

Développer la médiation documentaire numérique

sous la direction de Xavier Galaup

BA0 \#24 (2011)

Mener un projet international: bibliothèques françaises et coopération internationale

sous la direction de Raphaëlle Bats

BA0 \#23 (2011)

Créer des services innovants. Stratégies et répertoire d'actions pour les bibliothèques sous la direction de Marie-Christine Jacquinet

BA0 \#22 (2011)

Mener l'enquête! Guide des études de publics en bibliothèque

sous la direction de Christophe Evans
BA0 \#21 (2010)

Communiquer! Les bibliothécaires, les décideurs et les journalistes

sous la direction de Jean-Philippe Accart

BA0 \#20 (2010)

Mettre en œuvre un service de questionsréponses en ligne sous la direction de Claire Nguyen

BA0 \#19 (2010)

Numériser et mettre en ligne

sous la direction de Thierry Claerr et Isabelle Westeel

BA0 \#18 (2009)

Mettre en œuvre un plan de classement sous la direction de Bertrand Calenge

BA0 \#17 (2009)

Favoriser la réussite des étudiants sous la direction de Carine El BekriDinoird

BA0 \#16 (2008)

Gérer les périodiques

sous la direction de Géraldine Barron 
Secrétariat d'édition:

Silvia Ceccani

Mise en page:

Cédric Vigneault

Conception graphique:

atelier Perluette, 69001 Lyon.

< http://www.perluette-atelier.com >

Achevé d'imprimer en décembre 2015

imprimerie Bialec (Nancy) - $n^{\circ} 86969$

dépôt légal: $2^{\mathrm{e}}$ semestre 2015 